\title{
ESTUDO DE CABOS LIVREMENTE SUSPENSOS
}

\author{
Enga . MARIA BETÂNIA DE OLIVEIRA
}

\begin{abstract}
Dissertação apresentada à Escola de Engenharia de São Carlos, da Universidade de São Paulo, como parte dos requisitos para obtenção do Títuto de Mestre em Engenharia de Estruturas.
\end{abstract}

ORIENTADOR: Prof. Dr. Roberto L. A. Barbato 


\section{Oliveira, Maria Betânia de}

O48e Estudo de cabos livremente suspensos / Maria Betânia de Oliveira. - São Carlos, 1995.

$59 p$.

Dissertação (Mestrado) — Escola de Engenharia de São CarlosUniversidade de São Paulo, 1995.

Orientador: Prof.Dr. Roberto L. A. Barbato

1. Coberturas pênseis. 2. Cabos - Estruturas. I. Título. 
Aos meus pais,

meus exemplos de luta. 


\section{AGRADECIMENTOS}

Ao Prof. Roberto Luiz de Arruda Barbato pelo trabalho de orientação.

Ao Conselho Nacional de Desenvolvimento Científico e Tecnológico - CNPq, pela bolsa de estudo concedida.

Aos colegas, professores e funcionários do Departamento de Estruturas da EESC / USP que direta ou indiretamente tornaram possível a elaboração desta pesquisa. 


\section{SUMÁRIO}

RESUMO

ABSTRACT

\section{INTRODUÇÃO}

1.1 GENERALIDADES $\quad 01$

$\begin{array}{ll}1.2 \\ \text { DESCRIÇÃO SUCINTA DOS CAPÍTULOS } & 04\end{array}$

2 COBERTURAS PÊNSEIS - CONSIDERAÇÕES GERAIS

$\begin{array}{ll}2.1 \text { INTRODUÇÃO } & 06\end{array}$

$\begin{array}{ll}2.2 \text { SISTEMA ESTRUTURAL } & 06\end{array}$

2.2.1 CESTAS NÃO PROTENDIDAS 06

2.2.2 CESTAS PROTENDIDAS 08

$\begin{array}{ll}2.2 .3 \text { CABOS-TRELIÇA } & 09\end{array}$

2.3 SISTEMA VEDANTE E SUA ASSOCIAÇÃO COM O SISTEMA

ESTRUTURAL 11

2.4 EXEMPLOS DE OBROS CONSTRUÍDAS $\quad 12$

2.4.1 AEROPORTO INTERNACIONAL DE WASHINGTON 13

2.4.2 GINÁSIO DE ESPORTES GOVERNADOR EMÍLIO GOMES 16

\section{PROCESSO ANALÍTICO}

3.1 INTRODUÇÃO 23

3.2 EQUAÇÕES BÁSICAS DO ELEMENTO DE CABO 23

3.2.1 EQUAÇÕES DE EQUILÍBRIO

3.2.1.1 ELEMENTO DE CABO NA POSIÇÃO INICIAL 24

3.2.1.2 ELEMENTO DE CABO NA POSIÇÃO FINAL 24

3.2.2 RELAÇÃO ENTRE DEFORMAÇÕES E DESLOCAMENTOS 25

3.2.3 RELAÇÃO ENTRE ESFORÇOS E DEFORMAÇÕES 26 
3.3 CONFIGURAÇÃO INICIAL DE EQUILÍBRIO

3.4 CONFIGURAÇÃO FINAL DE EQUILÍBRIO 30

3.5 CONSIDERAÇÕES SOBRE AS CONDIÇÕES DE CONTORNO 35

\section{PROCESSO DOS DESLOCAMENTOS}

4.1 INTRODUÇ̃̃O 36

4.2 SISTEMA DE COORDENADAS 36

4.3 MATRIZ DE RIGIDEZ TANGENTE (INSTANTÂNEA) DO ELEMENTO 37

4.4 PROCESSO ITERATIVO DE RESOLUÇÃO 44

5 APLICAÇÃO NUMÉRICA

5.1 INTRODUÇÃO 45

5.2 CÁLCULO PELO PROCESSO ANALÍTICO 45

5.3 CÁLCULO PELO PROCESSO DOS DESLOCAMENTOS

6 VERIFICAÇÃO DE RESULTADOS MEDIANTE EXPERIMENTAÇÃo NUMÉRICA

6.1 INTRODUÇÃO

6.2 CARACTERÍSTICAS DOS CABOS ESTUDADOS 51

6.3 VERIFICAÇÃO DOS RESULTADOS DO PROCESSO DOS DESLOCAMENTOS 52

6.4 VERIFICAÇÃO DOS RESULTADOS DO PROCESSO ANALÍTICO 53

7 CONCLUSÃo

$\begin{array}{ll}\text { BIBLIOGRAFIA } & 57\end{array}$

APÊNDICES 


\section{RESUMO}

\section{OLIVEIRA, M. B. Estudo de cabos livremente suspensos. São Carlos,} 1995. Dissertação (Mestrado) - Escola de Engenharia de São Carlos, Universidade de São Paulo.

Modernamente os cabos vêm sendo empregados como elemento portante de coberturas de grandes vãos - as coberturas pênseis. É finalidade deste trabalho a apresentação da análise estática de cabos livremente suspensos, visando à sua aplicação nestas coberturas. Realiza-se o estudo de cabos - submetidos a carregamento estático uniformemente distribuído em todo o seu vão, a variações de temperatura e a deslocamentos de seus apoios - através do Processo Analítico (equações diferenciais) e do Processo dos Deslocamentos (rigidez tangente). Considera-se a não linearidade geométrica e admite-se a linearidade física. A comparação e análise dos resultados destes processos de cálculo são feitas através de experimentação numérica.

Palavras chaves: Coberturas Pênseis; Cabos - Estruturas. 


\section{ABSTRACT}

OLIVEIRA, M. B. Study of suspended cables. São Carlos, 1995. Dissertação (Mestrado) - Escola de Engenharia de São Carlos, Universidade de São Paulo.

Nowadays cables have been utilized as a resistant element in roofs of large dimensions, the hanging roofs. The objective of this work is to present the static analysis of suspended cables, aiming their application in roofs. A study of cables subjected to static uniform load distributed on the length, variations of temperature and displacements of the supports is done through Analytical Process (differential equations) and Stiffness Process (tangent stiffness). Geometrical non-linear analysis is allowed and physical linear analysis is admitted. The results are compared and analysed through numerical experimentation.

Keywords: Hanging Roofs; Suspended Cables; Tensile structures. 


\section{INTRODUÇÃO}

\subsection{GENERALIDADES}

Cabos são elementos estruturais usados em diferentes tipos de construções: são as principais peças portantes nas pontes pênseis, pontes estaiadas e nos teleféricos; são os elementos estruturais empregados para condução da energia elétrica nas linhas de transmissão, vencendo os vãos entre as torres da linha e, modernamente, vêm sendo empregados, também, como elemento portante de coberturas de grandes vãos - as coberturas pênseis - como exemplificam as obras a seguir.

A cobertura da Arena de Raleigh, na Carolina do Norte, em forma de superficie de dupla curvatura reversa, é suspensa de dois arcos de concreto armado de eixos parabólicos contidos em planos inclinados e que são suportados por pilares [BARBATO (1991)]. Outro exemplo é a cobertura do Aeroporto Internacional de Denver que consiste em uma série de tendas suportadas por fileiras de mastros. São empregados diversos tipos de elementos estruturais tais como cabos-treliça, cabos isolados e membrana de tecido translúcido [BERGER \& DE PADA (1992) ]. O Aeroporto Internacional de Washington é coberto por painéis pré-fabricados apoiados em cabos livremente suspensos ancorados em duas chapas engastadas em pilares. Após a colocação dos painéis, os sistemas estrutural e vedante são protendidos dando origem a uma casca pênsil cilíndrica protendida [ BARBATO (1991)]. Na Arena de Esportes de Atenas, uma cesta constituída por uma malha ortogonal de $4 \mathrm{~m} \times 4 \mathrm{~m}$ protendida, ancorada em uma viga de concreto armado, é 
coberta com uma lâmina ondulada de aço galvanizado [ ALESSI et al. (1979) ]. A cobertura do Armazém de Kogoshima é formada por uma estrutura de cabos e barras de aço dando mais rigidez à cobertura e distribuindo melhor os esforços na fundação [AWAGUCHI (1991)]. No Estádio de Esportes Karlsruhe na Alemanha foram usados os mesmos princípios da ponte pênsil. Uma treliça de aço suportando uma cobertura ondulada é pendurada de dois cabos suspensos em mastros. Estais são usados para transferir as forças dos cabos principais à fundação [SCHLAICH \& BERGERMANN (1984)]. No Estádio de Torino, construído para a Copa do Mundo de 1990, usa-se uma série de cabos-treliça radiais para cobrir a tribuna principal e redes de cabos na forma de parabolóide hiperbólico para cobrir as extremidades desta tribuna [MAJOWIECKI \& OSSOLA (1989) ]. A cobertura pênsil em cabos-treliça do Ginásio de Esportes de Herning, construído em Herning, Dinamarca, é suspensa de uma estrutura de concreto armado composta por dois anéis pré-fabricados e por 42 pilares que sustentam estes anéis - um superior e outro inferior. $O$ sistema estrutural da cobertura é formado por cabos-treliça radiais e por dois anéis de aço internos - um superior e outro inferior [BARBATO (1991)]. A cobertura do Ginásio de Esportes de Milão, construído no início dos anos 70, em forma de parabolóide hiberbólico de planta circular, é suspensa de uma estrutura de contorno formada por 38 grandes balanços de concreto armado, por peças em forma de losango que foram construídas com chapas de aço, e por um anel de aço em caixão que repousa sobre os losangos [ BARBATO (1991)]. No Ginásio de Esportes Governador Emílio Gomes cabos livremente suspensos são ancorados em dois anéis concêntricos. Sobre estes cabos são colocados painéis pré-fabricados que após protensão dão origem a uma casca pênsil de revolução protendida [ BARBATO (1991)]. O Ginásio de Voleibol de Beijing construído para os Jogos Asiáticos de 1990, apresenta diversos tipos de elementos estruturais de cobertura. Um deles, consiste de dois cabos isolados que suportam redes de cabos na forma de sela [SHEN et al. (1989)]. A cobertura do Ringue de Patinação de Munique consiste de uma cesta protendida de planta elíptica que suporta uma grelha de madeira revestida por tecido translúcido [SCHLAICH \& BERGERMANN(1984)]. Duas obras empregando coberturas suspensas foram construídas recentemente na China [ LAN (1986) ]. O Zhibo Sports Hall na província de Shandong emprega uma cobertura com sistema 
estrutural composto por cabos livremente suspensos e lajes pré-moldadas de concreto como vedação. O Sichuam Sports Hall utiliza cestas protendidas suspensas de arcos inclinados de concreto armado.

A autora desta dissertação sempre sentiu-se motivada a estudar coberturas de grandes vãos e optou começar com as coberturas pênseis, no intuito de contribuir para a sua divulgação e estudo no Brasil, onde não têm sido ainda, com raras exceções, empregadas como uma real opção de projeto. Neste sentido, é finalidade deste trabalho a apresentação de subsídios para a análise estática de cabos livremente suspensos visando à sua aplicação em sistemas estruturais de coberturas pênseis.

A análise estrutural de sistemas pênseis sob carregamento estático pode ser considerada, essencialmente, composta de dois problemas: determinação da configuração inicial de equilíbrio e cálculo dos deslocamentos resultantes das modificações do carregamento buscando-se determinar a posição final de equilíbrio.

Nas estruturas tracionadas é muito importante a determinação da configuração inicial de equilíbrio que geralmente depende das tensões atuantes. Muitas técnicas numéricas tem sido empregadas para obter a configuração inicial de equilíbrio como Técnicas da Mecânica do Contínuo e Métodos Iterativos de Elementos Finitos.

Em excelente artigo, HABER \& ABEL(1982a) revêem os principais métodos para o cálculo da configuração inicial de equilíbrio e mostram suas vantagens e desvantagens. Sugerem que o projetista tenha diversos métodos na disposição por não existir um método ótimo para todo tipo de projeto. Resultados aproximados de um procedimento devem ser usados como entrada de dados de um outro sistema para melhorar o projeto final.

A Computação Gráfica Iterativa esta sendo importante no campo da determinação da posição inicial de equilíbrio de estruturas tracionadas. HABER \& ABEL(1982b) apresentam um Sistema Gráfico Iterativo que combina diversos métodos através de uma base comum de dados para a solução do problema do equilíbrio inicial e MAJOWIECKI(1986) relata um sistema integrado de análise desenvolvido na Universidade de Bologna.

O estudo do comportamento estático de sistemas estruturais compostos por cabos é encontrado em muitas outras obras e pode-se citar KRISHMA(1978); 
IRVINE(1981); DEBS(1983); $\quad$ BUCHHOLDT(1985); OSHIMA(1987) BARBATO(1991).

Neste trabalho, empregando o Processo Analítico e o Processo dos Deslocamentos, faz-se a análise estática de cabos livremente suspensos - submetidos a carregamento uniformemente distribuído em todo o seu vão, a variações de temperatura e a deslocamentos dos seus apoios.

No Processo Analítico o estudo dos cabos livremente suspensos é realizado a partir de equações diferenciais em cujas resoluções são feitas algumas aproximações.

No Processo dos Deslocamentos a análise estática dos cabos é realizada discretizando-os em segmentos retilíneos ligados entre si por pontos nodais onde se supõem aplicadas as ações distribuídas.

Supondo conhecidas as ações aplicadas tem-se como objetivo a determinação dos esforços e deslocamentos de cabos livremente suspensos empregando os processos citados, a verificação da precisão dos resultados do Processo dos Deslocamentos com o aumento do número de segmentos e, também, a verificação das aproximações feitas no Processo Analítico.

Dois softwares desenvolvidos, um empregando Processo Analítico e o outro o Processo dos Deslocamentos, são utilizados na análise dos resultados destes processos de cálculo.

\subsection{DESCRIÇÃO SUCINTA DOS CAPÍTULOS}

O segundo capítulo apresenta conceitos básicos sobre as coberturas pênseis. São feitas considerações sobre as formas de sistemas estruturais, as formas de sistemas vedantes e a associação dos sistemas estruturais e vedantes. São mostradas duas coberturas pênseis compostas por cabos livremente suspensos, uma com planta retangular e outra com planta circular.

No terceiro capítulo é feito o estudo, pelo Processo Analítico, do comportamento estático de cabos livremente suspensos submetidos a carregamento uniformemente distribuído em todo o seu vão. Consideram-se as variações de 
temperatura e os deslocamentos dos seus apoios e, admite-se regime elástico linear com pequenas deformações.

O quarto capítulo é dedicado ao estudo de cabos livremente suspensos pelo Processo dos Deslocamentos. Utiliza-se a matriz de rigidez tangente e admite-se regime elástico linear com pequenas deformações.

No quinto capítulo, faz-se aplicação numérica para demonstrar a utilização dos processos apresentados nos capítulos 3 e 4 .

O sexto capítulo é voltado à ampla experimentação numérica com ambos os processos. É apresentado o universo que a experimentação numérica abrange e, também, a análise dos resultados obtidos.

No último capítulo emite-se parecer sobre a confiabilidade dos processos estudados. Dando introdução a este parecer, faz-se o fechamento desta dissertação trazendo à memória do leitor, em rápidas palavras, todo o seu conteúdo. Por fim, sugere-se temas para novas pesquisas que são uma evolução do assunto abordado neste trabalho.

Incluem-se quatro apêndices. $O$ Apêndice $A$ apresenta algumas propriedades dos cabos de aço nacionais. Os Apêndices $\mathrm{B}$ e $\mathrm{C}$ explicam os sistemas computacionais desenvolvidos com o emprego dos Processos Analítico e dos Deslocamentos, fornecendo, inclusive, os programas fonte. E o Apêndice D mostra os resultados da experimentação numérica com os dois processos de cálculo estudados. 


\section{COBERTURAS PÊNSEIS - CONSIDERAÇÕES GERAIS}

\subsection{INTRODUÇÃO}

A Cobertura Pênsil pode ser definida como um sistema construtivo que engloba um sistema estrutural suspenso e um sistema vedante que se apoia neste sistema estrutural.

O sistema estrutural suspenso pode ser entendido como aquele em que o principal elemento estrutural é o cabo de aço. Estes cabos são dispostos, basicamente, ou como cestas - que podem ser protendidas e não protendidas - ou como cabostreliça.

O sistema vedante compõe-se da vedação propriamente dita e de acessórios que têm a finalidade de fixar a vedação aos cabos de aço.

A associação dos sistemas estrutural e vedante deve ser estudada e definida de acordo com as características destes sistemas.

\subsection{SISTEMA ESTRUTURAL}

\subsubsection{CESTAS NÃO PROTENDIDAS}

As cestas não protendidas são geradas pela adequada associação de um conjunto de cabos livremente suspensos. A posição relativa dos cabos e sua configuração de equilíbrio definem a forma da superfície da cesta.

Dentre os diversos tipos de cestas não protendidas destaca-se o formado por cabos dispostos paralelamente que geram superfícies ou de simples curvatura ou de 
dupla curvatura. Cestas assim constituídas, com cabos suspensos de estruturas perimetrais, são empregadas frequentemente em coberturas de planta retangular.

Outro importante tipo de cesta não protendida é o constituído por cabos dispostos radialmente, ancorados em pontos situados ou não no mesmo nível, que geram superfícies de dupla curvatura. As cestas deste tipo são muito utilizadas em coberturas de planta circular. Nas mais comuns, os cabos radiais são ancorados em um anel externo que se apoia em estruturas perimetrais e em um anel interno que fica suspenso dos cabos no centro da área coberta.

Também são usadas cestas cujos cabos radiais ancoram-se em um anel externo e na extremidade superior de uma torre central. A cesta gerada, neste caso, tem a forma de uma superfície de revolução de dupla curvatura reversa. Evidentemente, cestas de cabos radiais podem ser usadas em coberturas de planta elíptica ou de planta com forma de setor de círculo ou de setor de coroa circular.
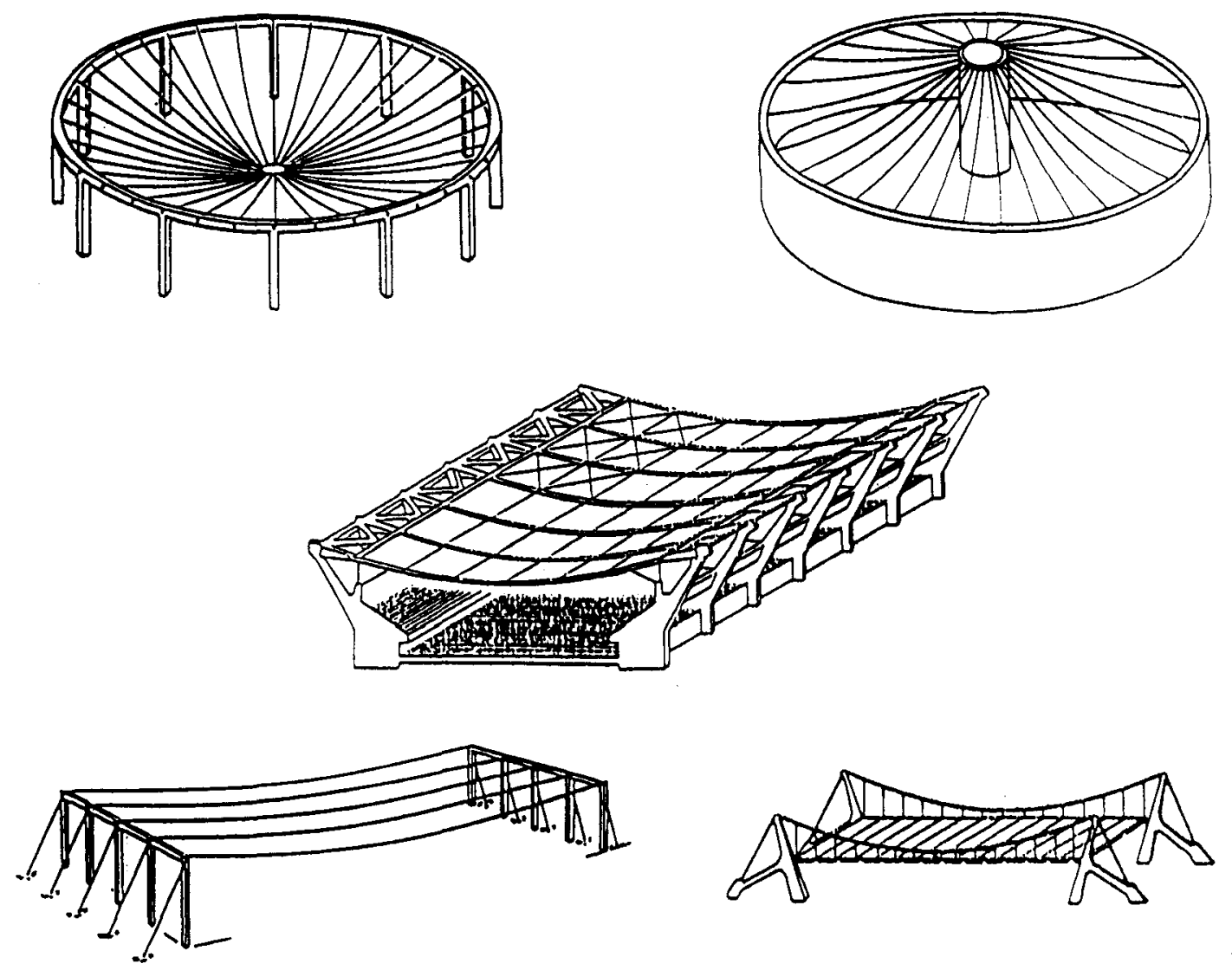

Figura 2.2.1.1 - Esquem.ıs de cestas não protendidas. 


\subsubsection{CESTAS PROTENDIDAS}

As cestas protendidas são estruturas que resultam de cestas não protendidas em cujos cabos, adequadamente dispostos, aplicam-se esforços prévios de tração.

Dentre os diversos tipos de cestas protendidas destaca-se o constituído por duas familias de cabos que, transversalmente associados formando malhas retangulares, geram uma superfície de dupla curvatura reversa que pode ser assimilada a um parabolóide hiperbólico. A protensão deste tipo de cesta - empregado com frequência em coberturas de planta circular e elíptica - é aplicada mediante o tracionamento dos cabos de uma ou de ambas famílias.

Outro interessante tipo de cesta protendida é o que pode ser obtido associando-se duas famílias de cabos de modo a compor uma estrutura que tem a forma de uma superfície de revolução de dupla curvatura reversa. Neste caso, um mastro central sustenta a cesta cujos cabos, dispostos segundo os meridianos e os paralelos, formam malhas trapezoidais de dimensões variáveis. A protensão da cesta pode ser introduzida, por exemplo, pelo tracionamento dos cabos meridianos.
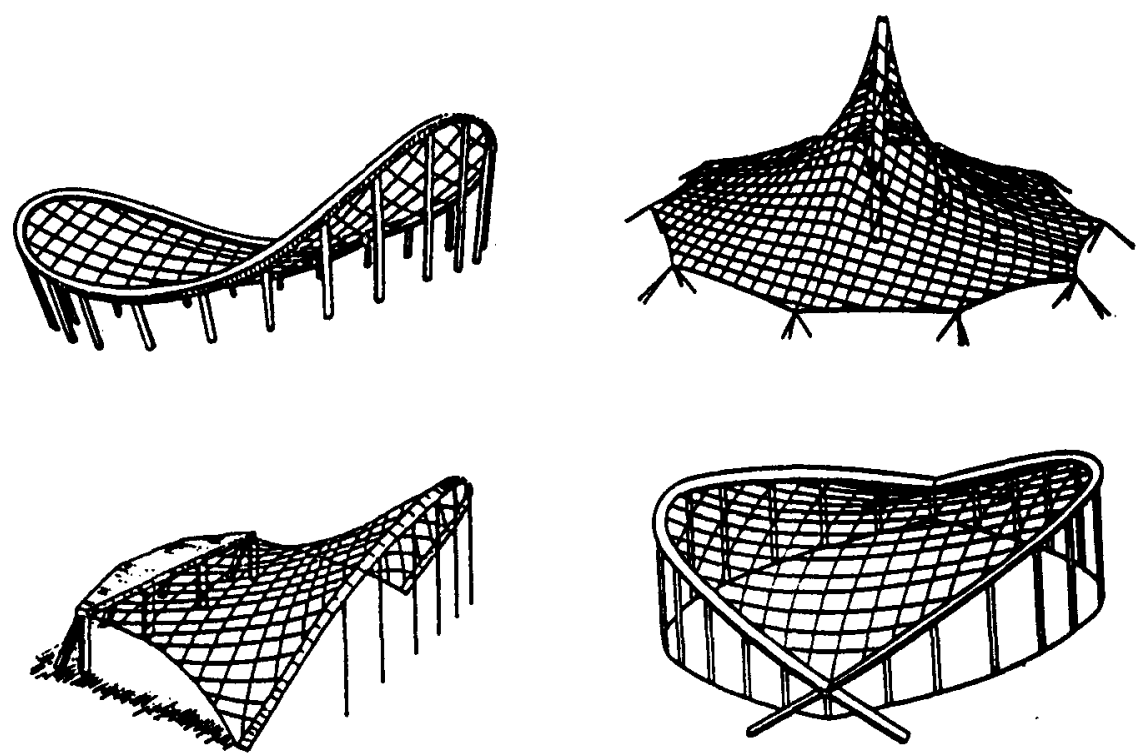

Figura 2.2.2.1 - Esquemas de cestas protendidas. 


\subsubsection{CABOS-TRELIÇA}

Constituídos, essencialmente, por dois cabos e por um conjunto de hastes que os conectam, os cabos-treliça são estruturas protendidas que têm a forma de uma treliça plana de banzos não paralelos. Os cabos compõem os banzos da treliça e as hastes a sua alma.

Os cabos-treliça podem ser considerados, de acordo com a configuração de seus dois cabos, ou bicôncavos, ou biconvexos ou ainda mistos. Os cabos-treliça mistos são aqueles que têm um trecho central biconvexo e dois trechos extremos bicôncavos. As hastes que conectam os dois cabos, dispostas paralelamente ou em diagonal, ficam sujeitas a esforços de tração nos cabos-treliça bicôncavos e a esforços de compressão nos biconvexos. Nos cabos mistos as barras do trecho central ficam comprimidas e as dos trechos extremos tracionadas.

Para compor o sistema estrutural, os cabos-treliça podem ser dispostos ou paralelamente, ou radialmente ou ainda transversalmente.

Cabos-treliça paralelos, nivelados ou desnivelados são correntemente empregados em coberturas de planta retangular. Suspensos de estruturas situadas no perímetro - geralmente pilares engastados na fundação ou articulados e espiados - os cabos-treliça formam duas superfícies, que dependendo da configuração de cada um de seus cabos, podem ser de simples ou de dupla curvatura. Observe-se que cabos paralelos também podem ser usados em coberturas de planta circular ou elíptica.

Cabos-treliça dispostos radialmente são particularmente utilizados em coberturas de planta circular ou em forma de setor de círculo. Nas de planta circular mais comuns os cabos-treliça são ancorados em anéis (ou anel) externos que se apoiam em estruturas de contorno e em anéis (ou anel) internos que ficam suspensos dos cabos no centro da área coberta. $O$ emprego de torre central para a ancoragem dos cabos-treliça pode representar interessante alternativa para as coberturas de planta circular.

Reticulados tridimensionais constituídos por cabos-treliça dispostos transversalmente e com igual espaçamento nas duas direções, podem ser utilizados 
em coberturas de planta circular ou elíptica. Nas de planta circular, por exemplo, os cabos-treliça são ancorados em um ou em dois anéis externos e geram duas superfícies de dupla curvatura de mesmo sentido.

Protende-se o cabo-treliça ou aplicando-se esforços de tração em um ou em ambos os cabos, ou aplicando-se esforços de tração ou de compressão nas barras, ou ainda mediante a aplicação simultânea de esforços nos cabos e nas barras.
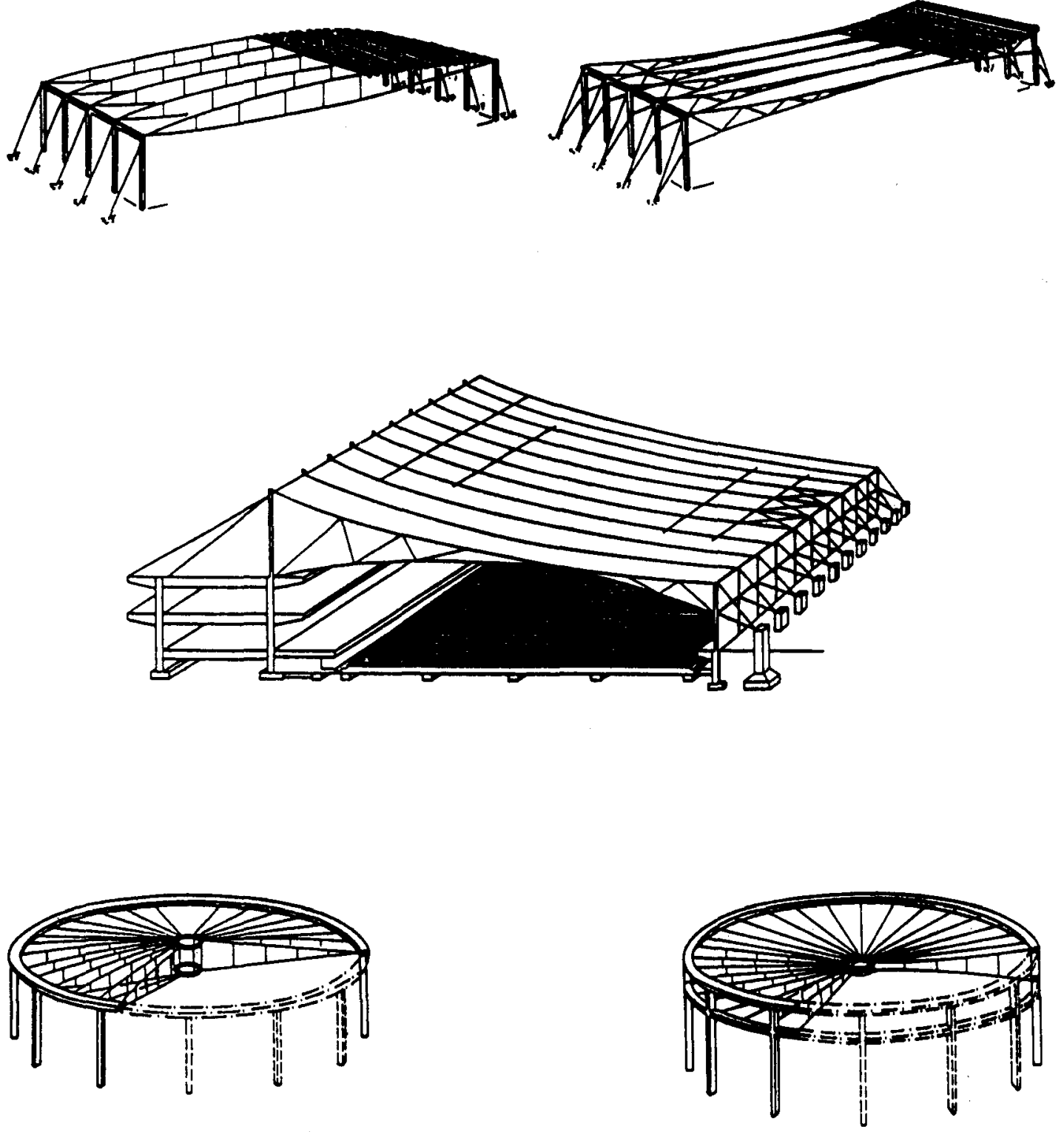

Figura 2.2.3.1 - Esquemas de sistemas estruturais compostos por cabos-treliça. 


\subsection{SISTEMA VEDANTE E SUA ASSOCIAÇÃO COM O SISTEMA ESTRUTURAL}

O sistema vedante compõe-se da vedação propriamente dita e de acessórios que têm a finalidade de fixar a vedação aos cabos de aço. Os elementos de vedação podem ser leves ou pesados. São constituídos por diversos tipos de materiais tais como aço, concreto armado, argamassa armada, madeira, plástico, fibra de vidro, etc. Para fixar a vedação no sistema estrutural empregam-se grampos, ganchos, parafusos, perfis e cantoneiras como mostram, por exemplo, as figuras seguintes.

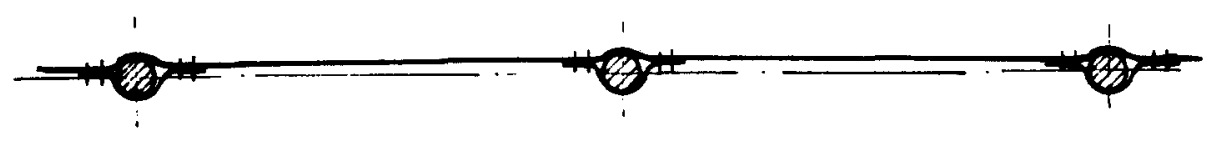

Figura 2.3.1 - Fixação de lâminas ou de náilon, ou de plástico.
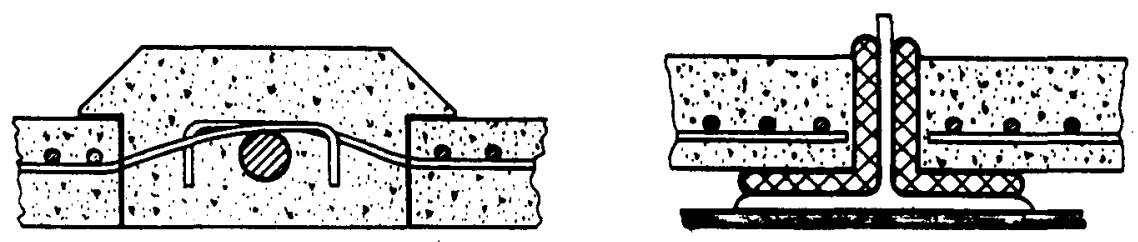

Figura 2.3.2 - Fixação das placas de argamassa ou de concreto armado.
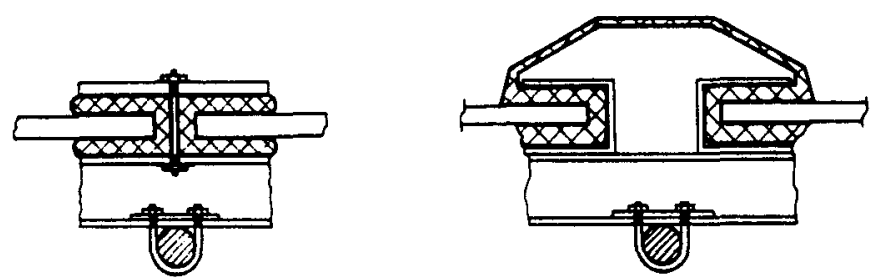

Figura 2.3.3 - Fixação de chapas lisas.
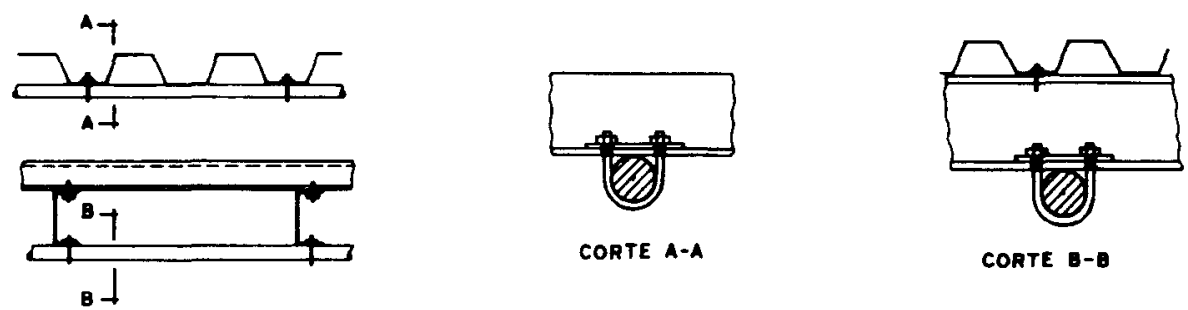

Figura 2.3.4 - Fixação de telhas de aço e de alumínio. 
Descritos os sistemas estruturais e vedantes que têm sido utilizados com maior frequência, é necessário que se façam algumas considerações a respeito de sua associação tendo em vista a construção de coberturas pênseis.

Cestas não protendidas são sistemas estruturais que - em consequência do peculiar comportamento mecânico do cabo livremente suspenso - tendem a sofrer grandes deslocamentos. Assim sendo, percebe-se claramente que este tipo de sistema estrutural deve ser associado a um sistema vedante que, especialmente projetado, mobilize mecanismos resistentes que se opondo às ações atuantes - principalmente as assimétricas oriundas do vento - limitem os deslocamentos da cesta a valores compatíveis com o uso da cobertura.

Dos sistemas vedantes já empregados, o mais simples é o constituído por placas pré-moldadas de concreto armado, por acessórios que as fixam nos cabos e por material selante, rígido ou deformável, que, após o assentamento das placas, é introduzido nas juntas. Quando se emprega concreto como material selante, por exemplo, as placas do sistema ficam rigidamente ligadas dando origem a uma cobertura em casca pênsil de elementos pré-fabricados. Aplicando-se uma carga sobre as placas antes da concretagem das juntas e retirando-a após o endurecimento do concreto introduzido, obtém-se uma cobertura pênsil em casca protendida de elementos pré-moldados. Coberturas assim construídas já foram empregadas, com sucesso, em pelo menos três obras brasileiras.

Cestas protendidas e cabos-treliça são estruturas cujo desempenho depende, essencialmente, de sua configuração de equilíbrio e da intensidade de protensão atuante em seus elementos. Adequadamente projetados quanto à forma e quanto à protensão resultam consideravelmente rígidas e podem ser associadas a sistemas de baixo peso próprio e que tenham apenas a função de vedar.

\subsection{EXEMPLOS DE OBRAS CONSTRUÍDAS}

No capítulo anterior, citaram-se rapidamente 13 obras para dar uma idéia sobre o que existe de coberturas pênseis. Agora, descrevem-se com maiores detalhes duas entre aquelas que empregam os cabos livremente suspensos e que seus sistemas 
estrutural e vedante são associados de modo a dar origem a uma casca pênsil protendida - duas obras que estão mais próximas do estudo desenvolvido neste trabalho.

\subsubsection{AEROPORTO INTERNACIONAL DE WASHINGTON}

A cobertura pênsil do terminal é suspensa de uma estrutura formada por 32(2x16) pilares de concreto armado de seção variável e por duas chapas, também de concreto armado, onde são ancorados os cabos da cobertura. Os pilares - 16 com $19,2 \mathrm{~m}$ de altura e os outros $16 \mathrm{com} 13,1 \mathrm{~m}$ de altura - são espaçados de $12,2 \mathrm{~m}$ e engastados nas fundações. Os cabos da cobertura, em número de 128 , têm diâmetro de $2,54 \mathrm{~cm}$ e são de aço de alta resistência. Como vedação empregaram-se painéis nervurados de concreto leve de $2,64 \times 1,80 \mathrm{~m}^{2}$, com mesa de $2,54 \mathrm{~cm}$ de espessura e nervuras de $5 \times 19 \mathrm{~cm}^{2}$. A armadura das nervuras foi deixada saliente de modo a formar ganchos para 0 apoio dos painéis nos cabos.

Após o assentamento dos painéis de vedação sobre os cabos portantes, fixaram-se as formas e posicionaram-se as armaduras das juntas. A concretagem foi feita simultaneamente com a aplicação da carga de protensão. Endurecido o concreto lançado, a carga de protensão - obtida por meio de sacos de areia e aplicada à borda dos painéis — foi retirada resultando em uma casca pênsil cilíndrica protendida.

O projeto arquitetônico do terminal foi elaborado por Eero Saarinen. O projeto estrutural é de autoria de Amman e Whiteney. A área coberta do terminal, construído no início da década de 1960 , é de $8350 \mathrm{~m}^{2}$.

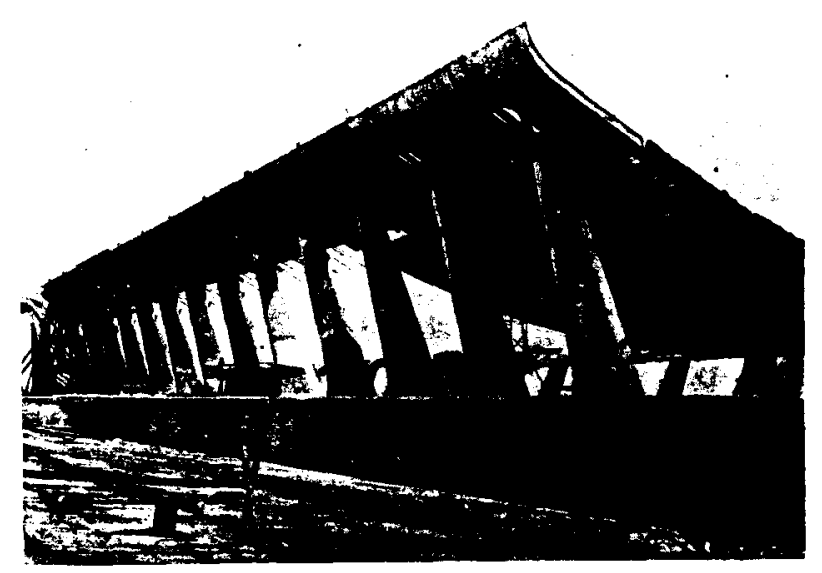

Figura 2.4.1.1 - Colocação dos cabos e dos painéis de vedação. 


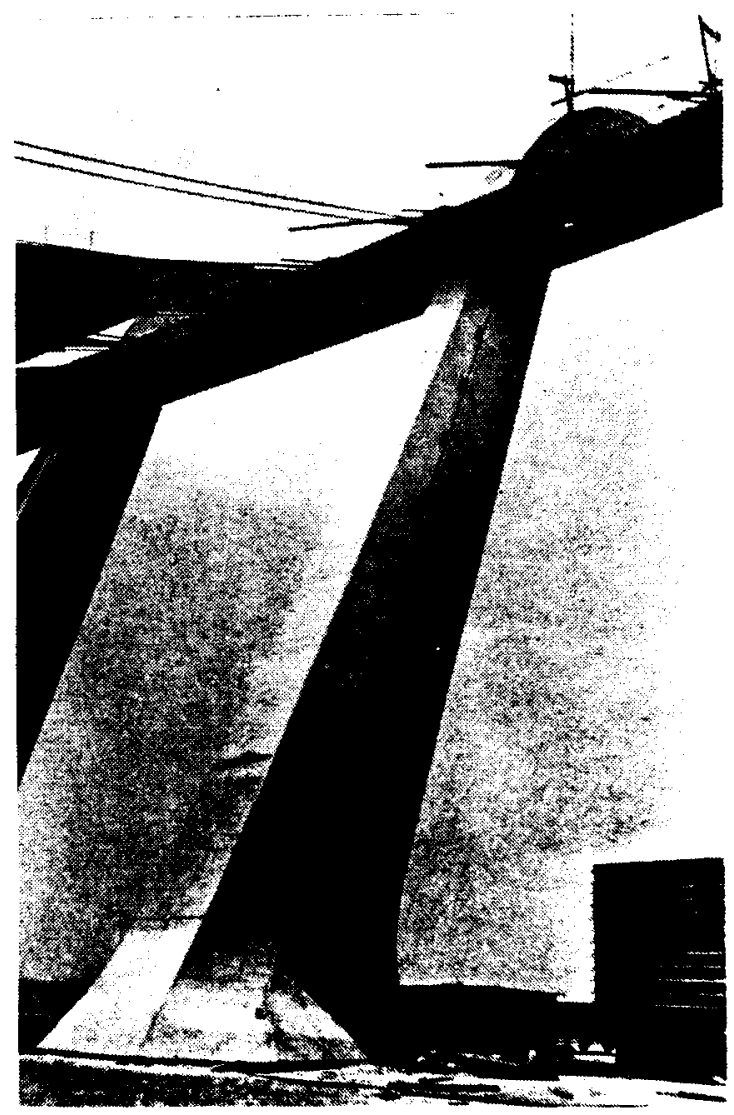

Figura 2.4.1.2 - Pilares e chapas onde são ancorados os cabos.

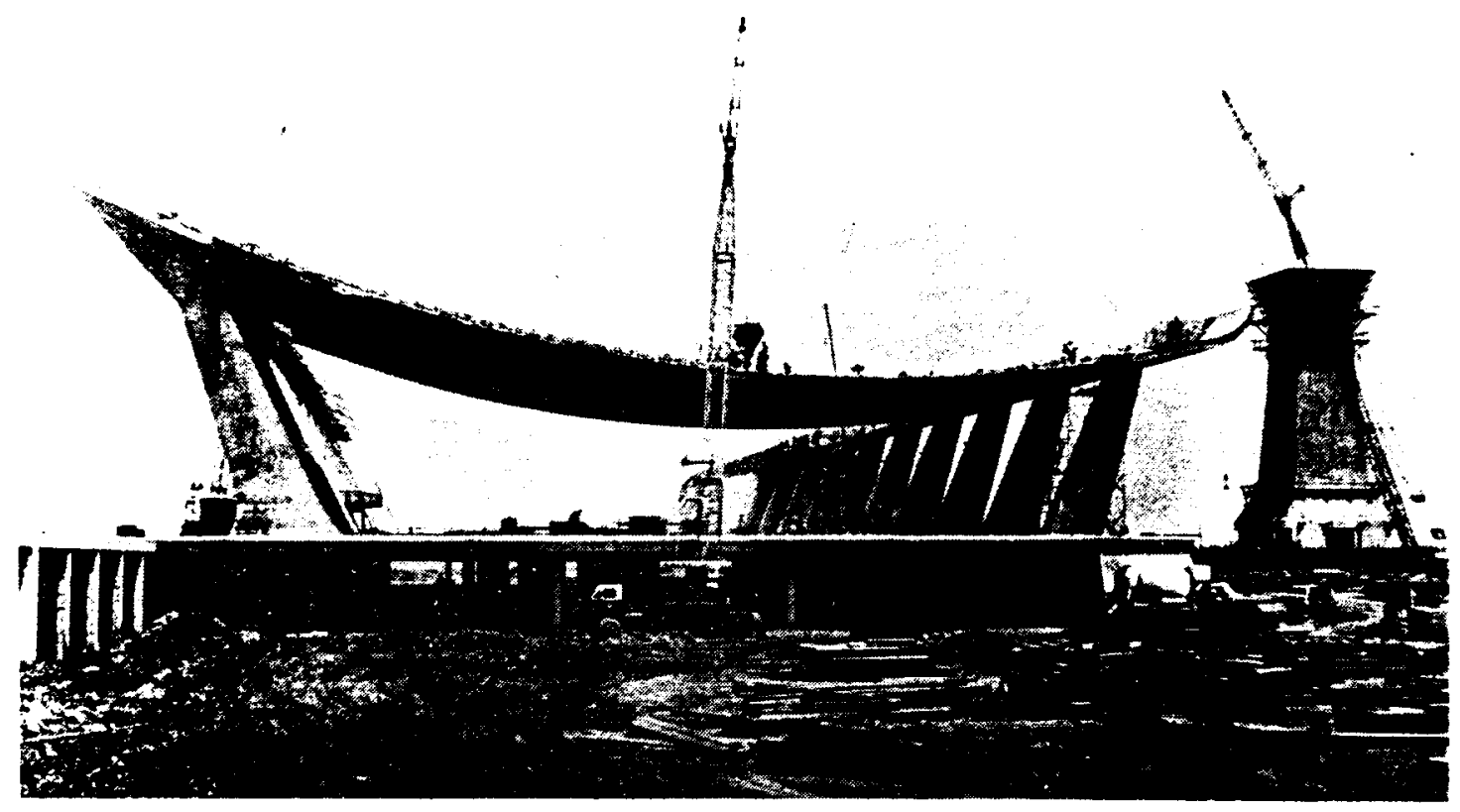

Figura 2.4.1.3 - Vista lateral da obra. 


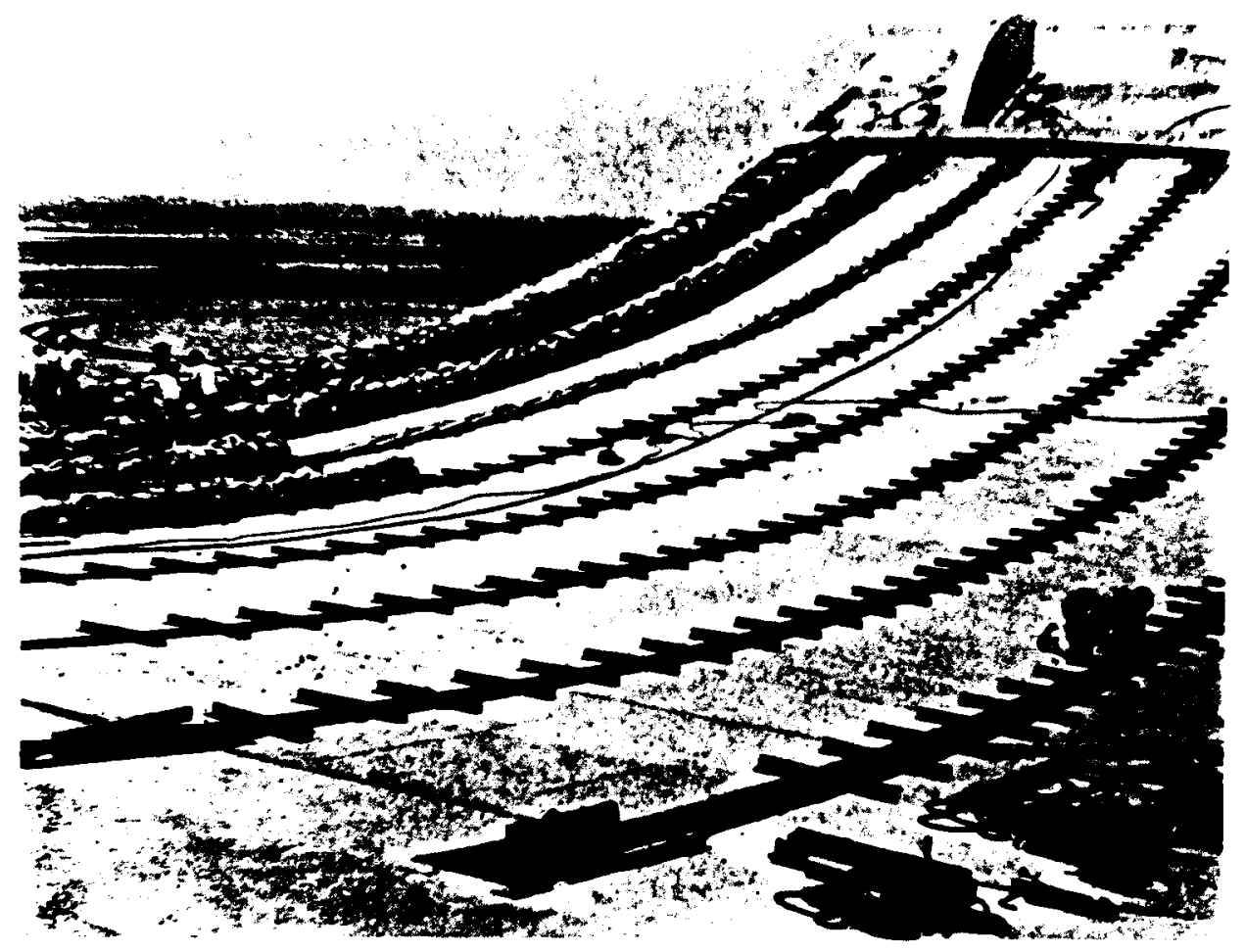

Figura 2.4.1.4 - Operação simultânea de concretagem das juntas e aplicação da protensão.

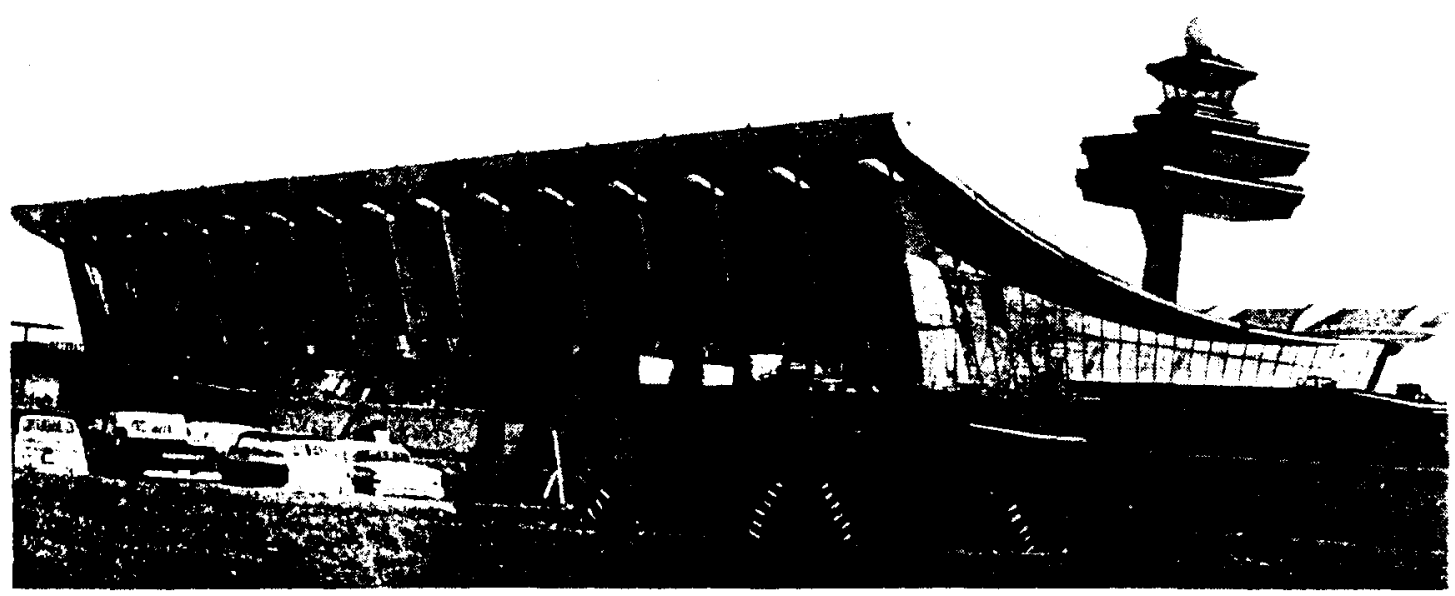

Figura 2.4.1.5 - Obra em fase final de construção. 


\subsubsection{GINÁSIO DE ESPORTES GOVERNADOR EMÍLIO GOMES}

O teto suspenso com $60 \mathrm{~m}$ de diâmetro do Ginásio de Esportes Governador Emílio Gomes em Rolândia, Estado do Paraná, foi construído em 1974.

Esta cobertura pênsil de planta circular em casca protendida de revolução é composta de dois anéis concêntricos, de uma cesta de cabos radiais ancoradas nos anéis e de elementos de vedação — placas pré-moldadas - que se apoiam nos cabos que formam a cesta.

Uma carga de protensão é aplicada, por meio de tijolos e sacos plásticos contendo água ou areia, após o assentamento de todas as placas e retirada depois do endurecimento do concreto colocado nas juntas - obtendo-se uma casca pênsil de rotação protendida.

O projeto arquitetônico foi elaborado por BATAGLIA \& WELKER. O projeto estrutural é de autoria de BARBATO \& MARTINELLI.

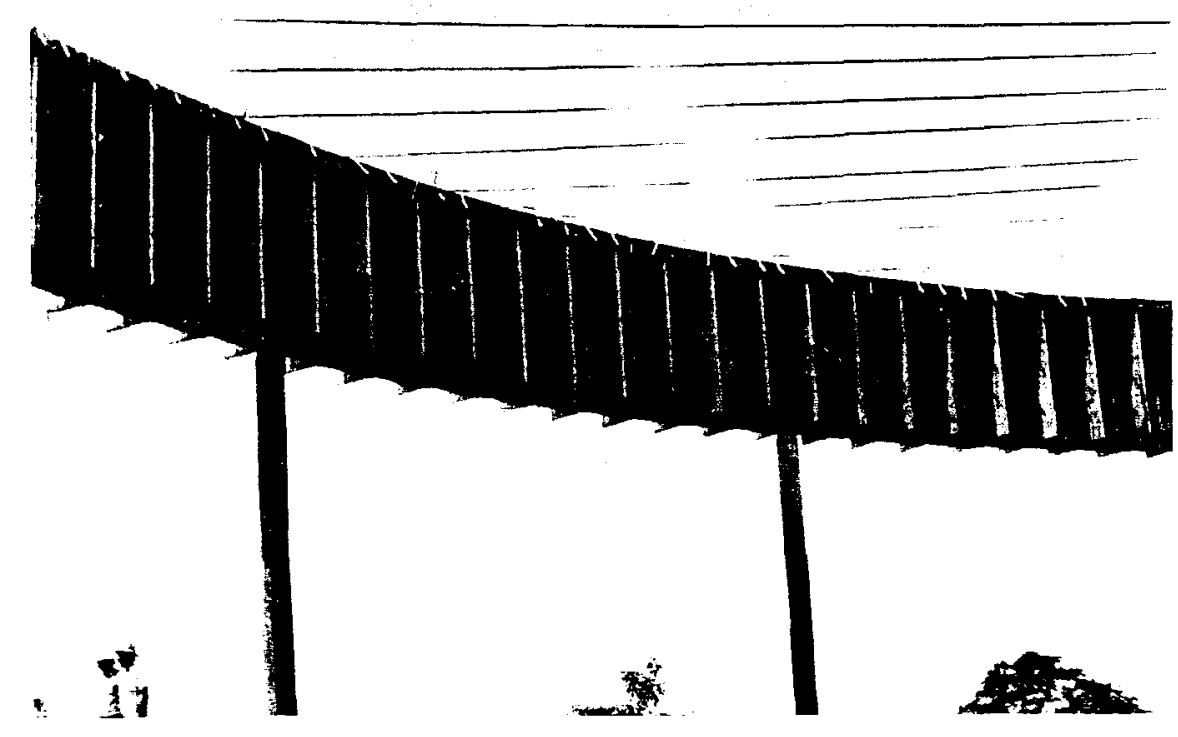

Figura 2.4.2.1 - Anel exerno com os cabos ancorados. 


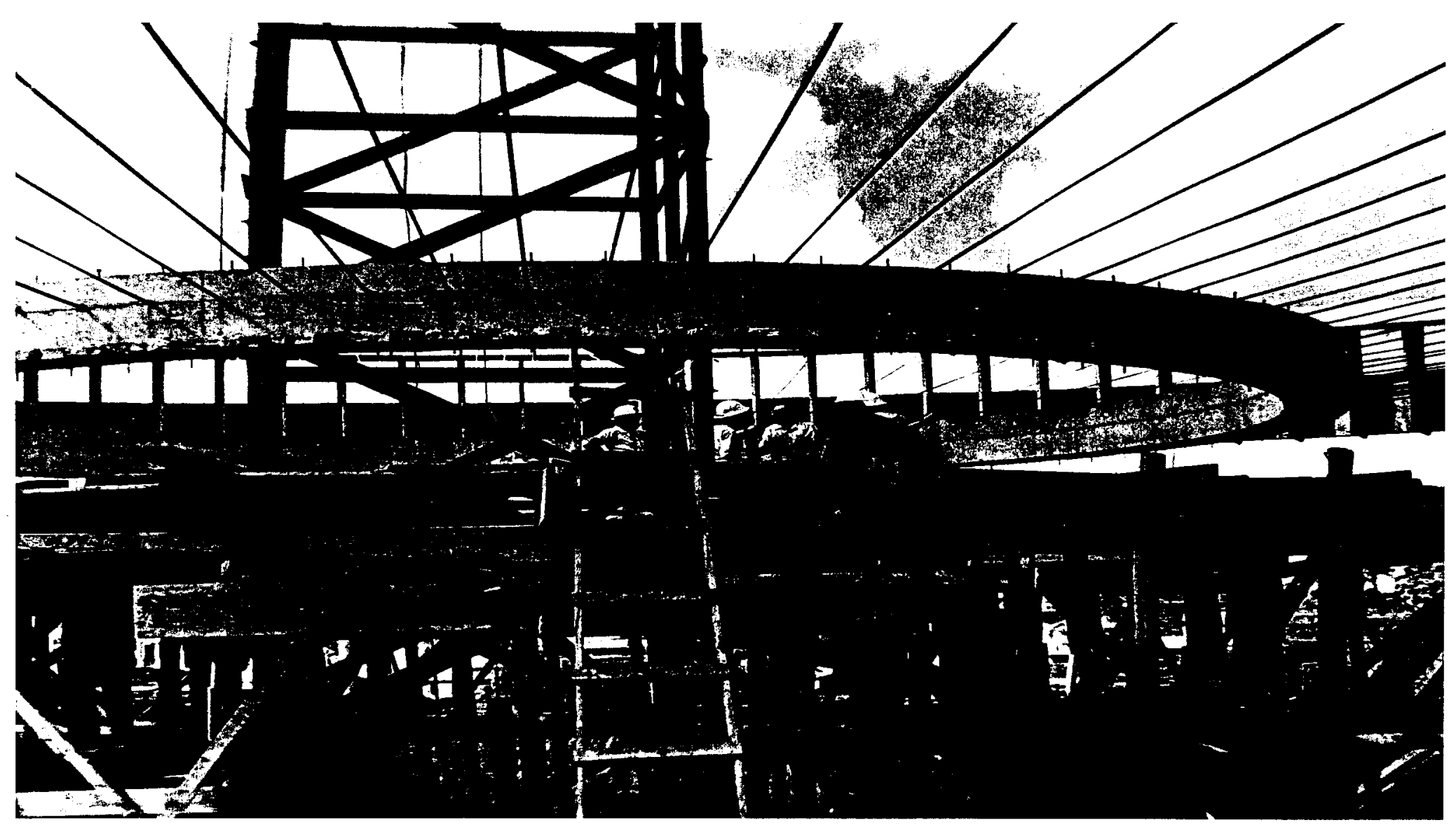

Figura 2.4.2.2 - Cimbramento central e anel interno suspenso dos cabos. 


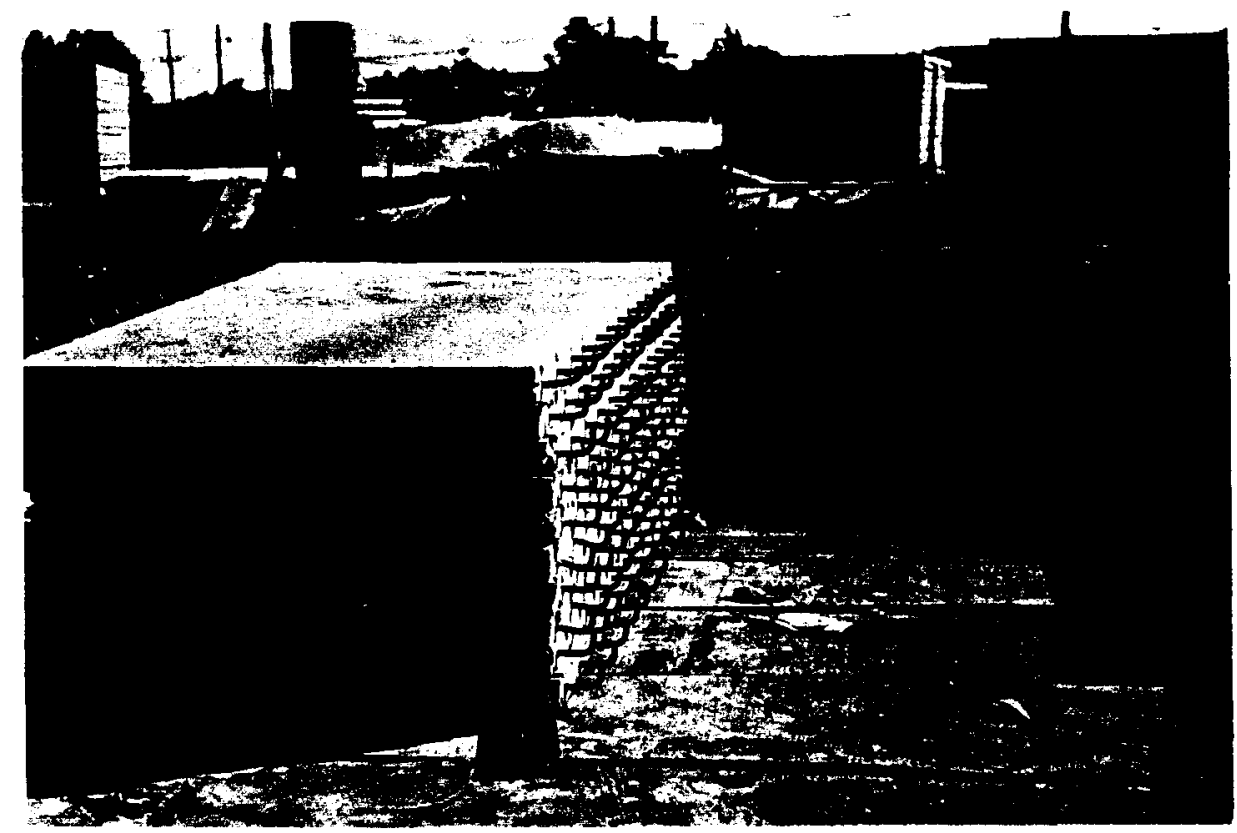

Figura 2.4.2.3 - Placas pré-moldadas de concreto armado ( $4 \mathrm{~cm}$ de espessura).

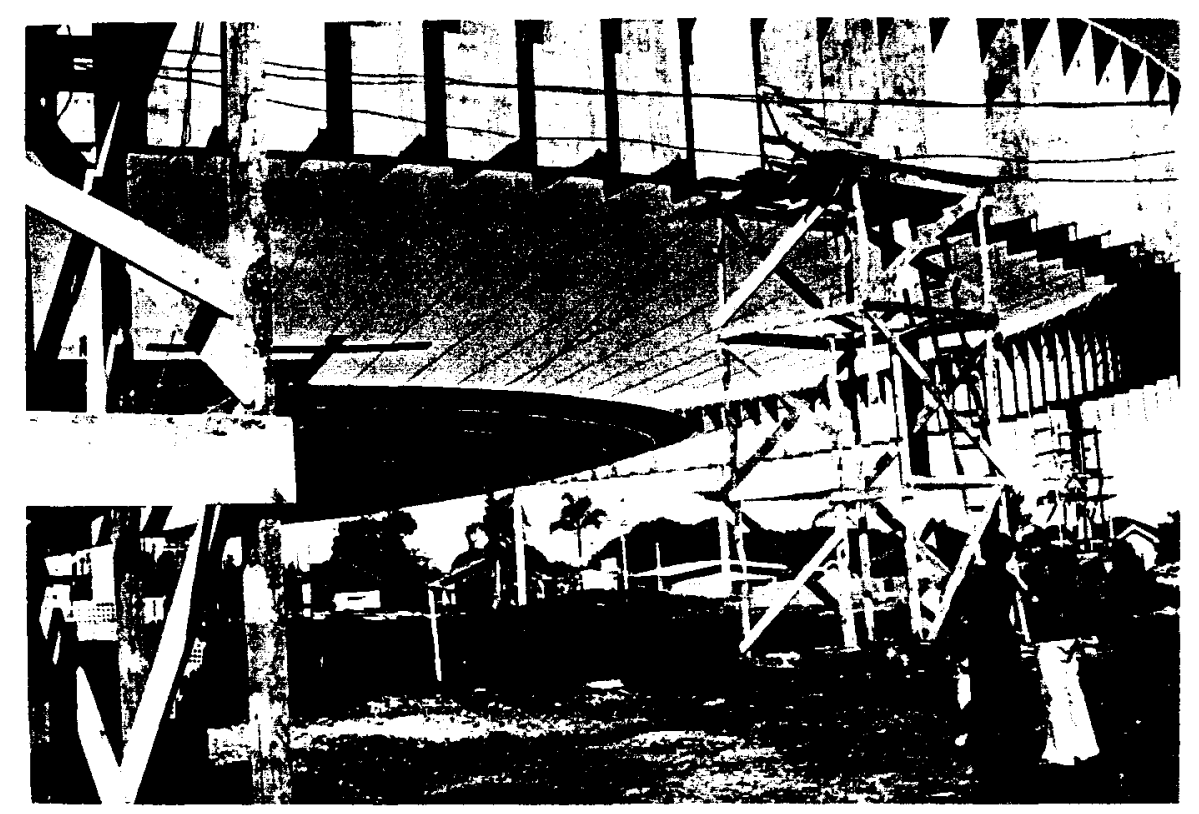

Figura 2.4.2.4 - Colocação das placas de vedação sobre os cabos. 


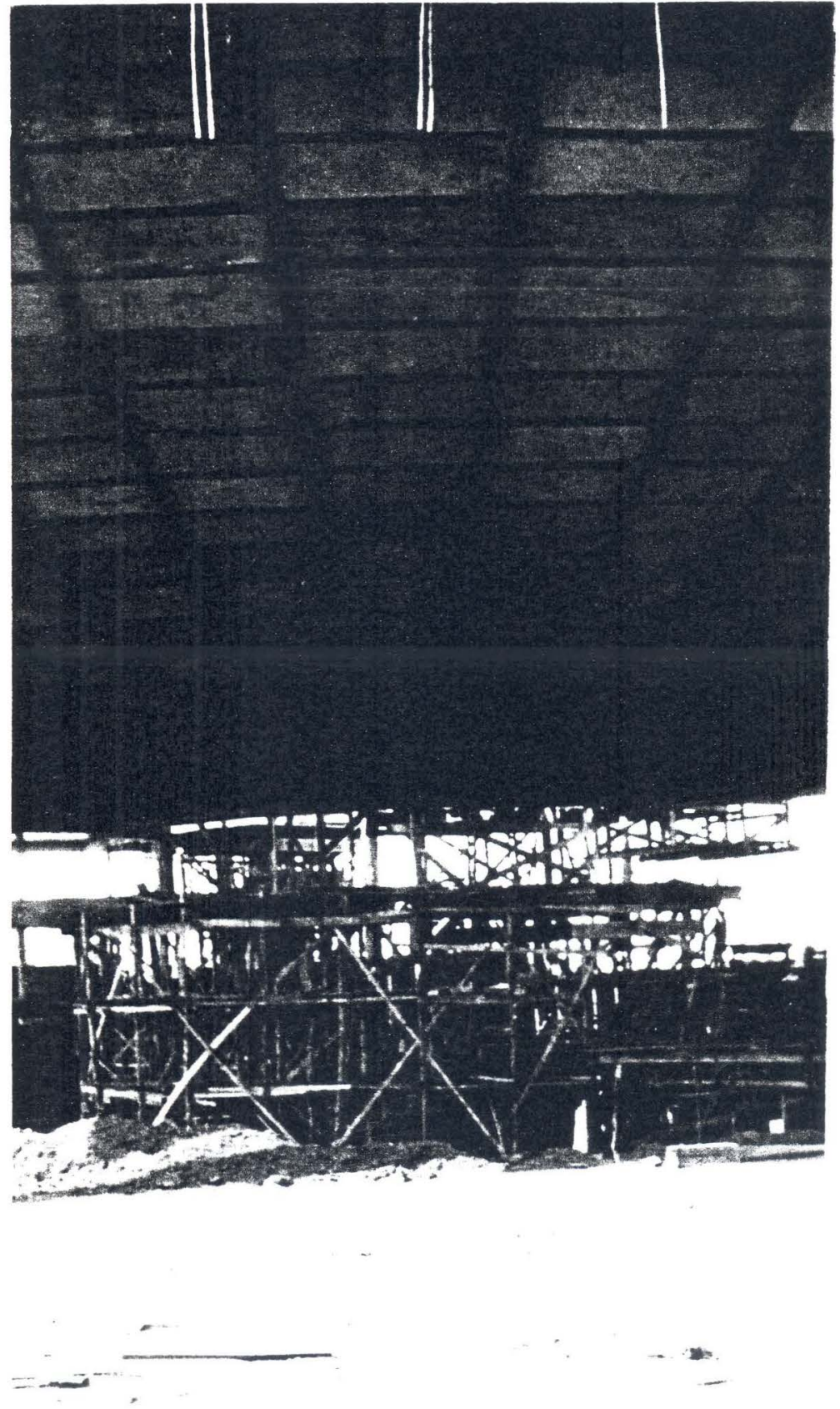

Figura 2.4.2.5 - Vista inferior da cobertura após o assentamento das placas . 


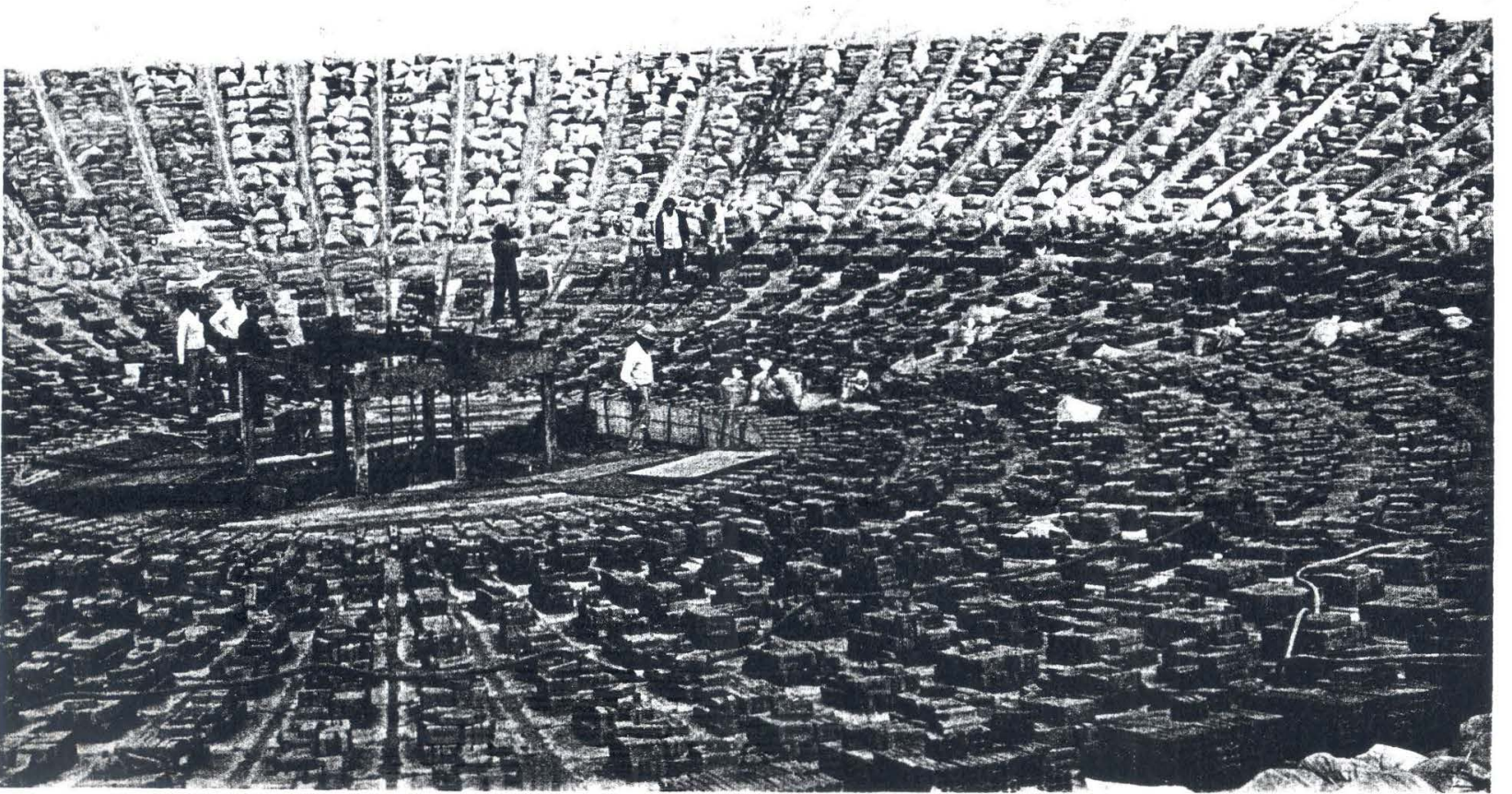

Figura 2.4.2.6 - Cobertura com a carga de protensão. 


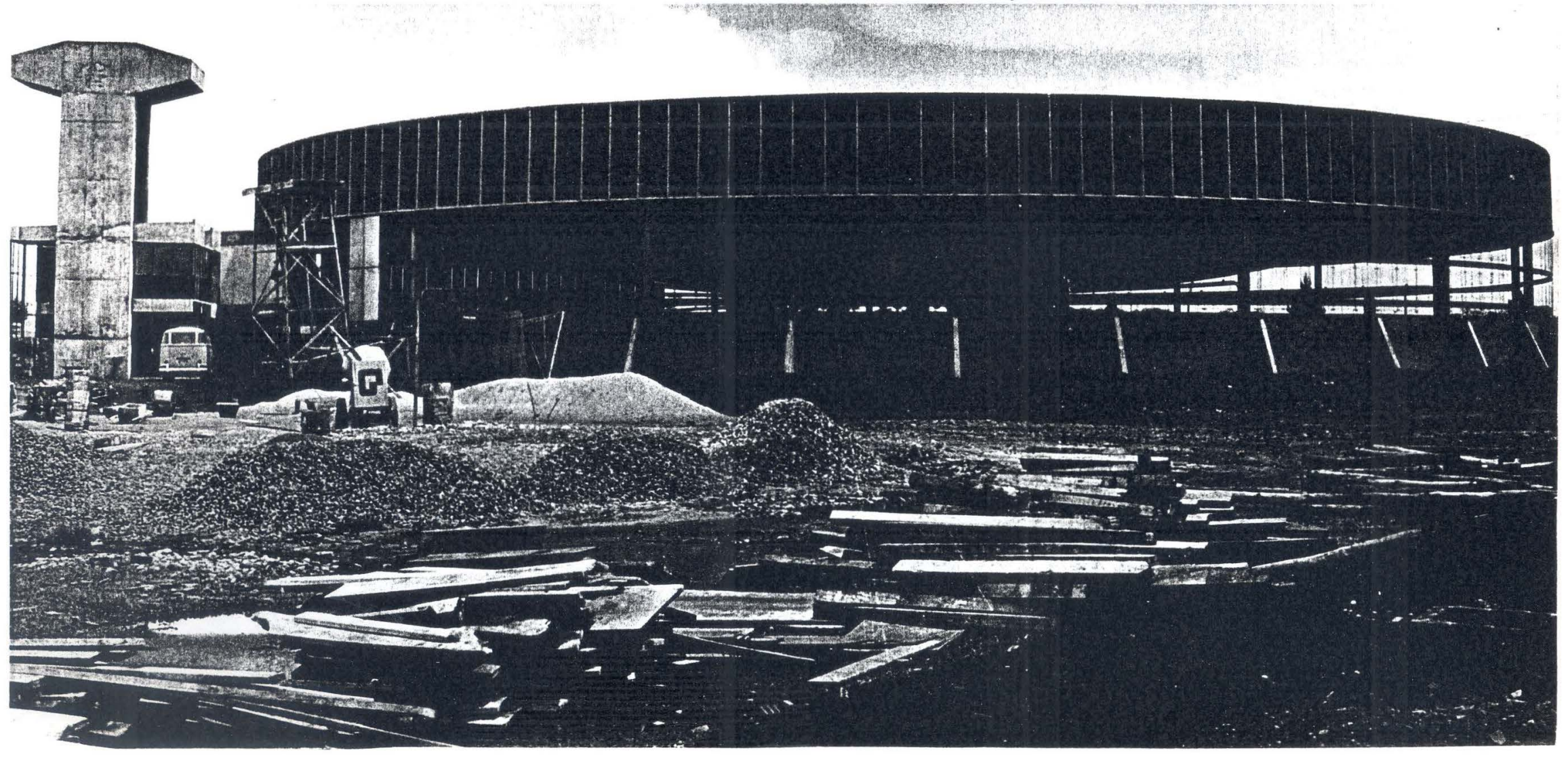

Figura 2.4.2.7 - Vista global da cobertura. 


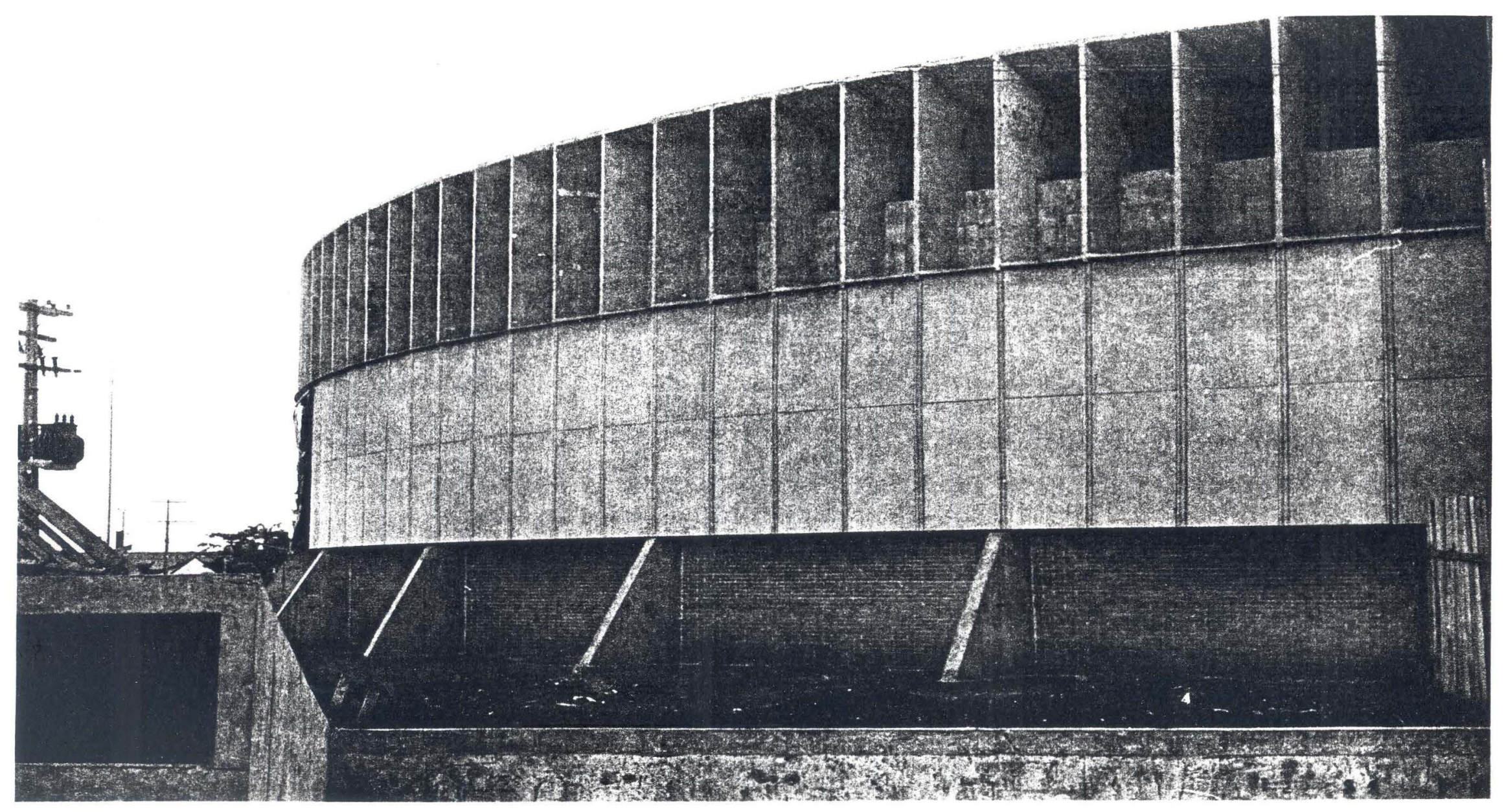

Figura 2.4.2.8 - Vedação lateral em alvenaria placas de cimento amianto. 


\section{PROCESSO ANALÍTICO}

\subsection{INTRODUÇÃO}

No Processo Analítico o cabo é estudado a partir de equações diferenciais que relacionam tensões (esforços), deformações e deslocamentos.

Essas equações resolvidas levam às expressões que permitem analisar o cabo solicitado por diversos tipos de carregamento. No entanto, o estudo que se apresenta a seguir contempla apenas cabos solicitados por carregamento uniformemente distribuído ao longo de todo o seu vão. Consideram-se, também, os efeitos das variações de temperatura e dos deslocamentos dos apoios.

Admite-se regime elástico linear com pequenas deformações e a invariabilidade da área da seção transversal do cabo.

\subsection{EQUAÇÕES BÁSICAS DO ELEMENTO DE CABO}

Faz-se, neste item, a dedução das equações que regem o comportamento estático dos cabos livremente suspensos.

\subsubsection{EQUAÇÕES DE EQUILÍBRIO}

Nas equações seguintes, as condições de equilíbrio do elemento de cabo são satisfeitas nas posições inicial e final. 


\subsubsection{ELEMENTO DE CABO NA POSIÇÃO INICIAL}

As equações de equilibrio de forças e momentos para um elemento de comprimento inicial ds, figura 3.2.1.1.1, são:

$\mathrm{dH}=0$

$\mathrm{dV}+\mathrm{pdx}=0$

$V d x-H d y-p d x\left(\frac{d x}{2}\right)=0$

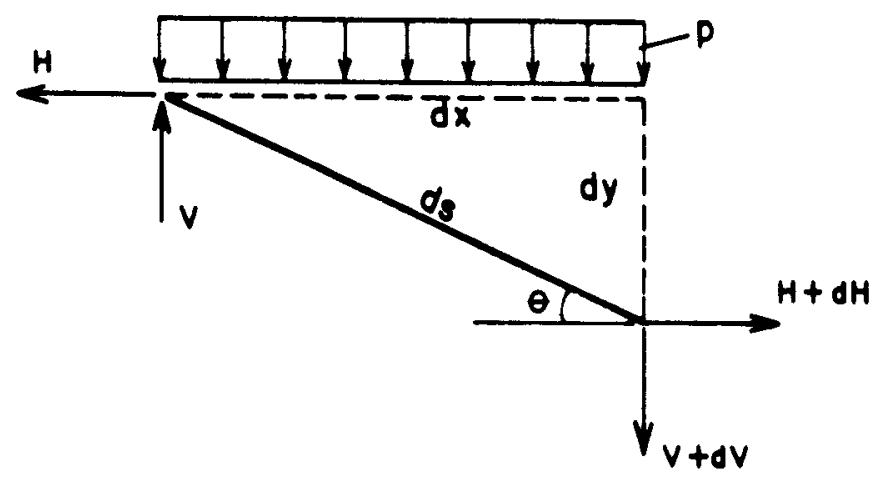

Figura 3.2.1.1.1 - Elemento de cabo na posição inicial.

\subsubsection{ELEMENTO DE CABO NA POSIÇÃO FINAL}

Analogamente ao item anterior, as equações de equilíbrio aplicadas ao elemento de comprimento final $\mathrm{d} \overline{\mathrm{s}}$, figura 3.2.1.2.1, são:

$$
\begin{aligned}
& d \bar{H}=0 \\
& \bar{V}-\bar{p}(d x+d u)-(\bar{V}+d \bar{V})=0 \\
& \bar{V}(d x+d u)-\bar{H}(d y+d v)-\bar{p} \frac{(d x+d u)^{2}}{2}=0
\end{aligned}
$$




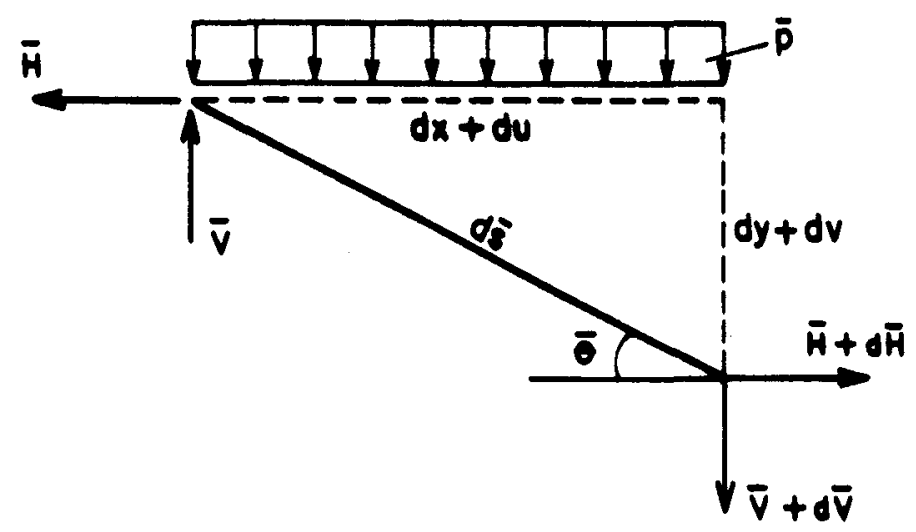

Figura 3.2.1.2.1 - Elemento de cabo na posição final.

\subsubsection{RELAÇÃO ENTRE DEFORMAÇÕES E DESLOCAMENTOS}

Os comprimentos ds e $\mathrm{d} \overline{\mathrm{s}}$, figura 3.2.2.1, podem ser expressos por:

$$
\begin{aligned}
& d s^{2}=d x^{2}+d y^{2} \\
& d \bar{s}^{2}=(d x+d u)^{2}+(d y+d v)^{2}
\end{aligned}
$$

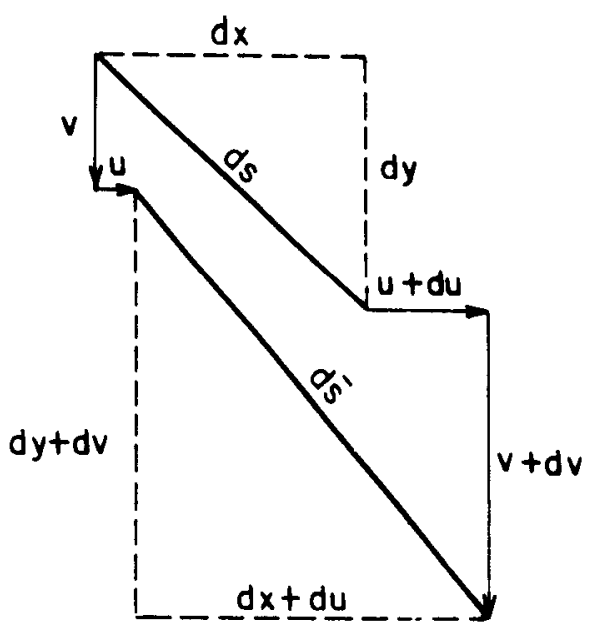

Figura 3.2.2.1 - Elementos de cabo nas posições inicial e final. 
Definindo-se a deformação específica $\varepsilon$ por

$\varepsilon=\frac{\mathrm{d} \overline{\mathrm{s}}-\mathrm{ds} \mathrm{s}_{0}}{\mathrm{ds}_{0}}$

e combinando-se (3.2.2.1), (3.2.2.2) e (3.2.2.3), obtém-se a equação (3.2.2.4) que relaciona deformações e deslocamentos:

$\varepsilon=\sqrt{1+\frac{2 u^{\prime}+u^{\prime 2}+2 y^{\prime} v^{\prime}+v^{\prime 2}}{1+y^{\prime 2}}}-1$

\subsubsection{RELAÇÃO ENTRE ESFORÇOS E DEFORMAÇÕES}

A figura 3.2.3.1 mostra um elemento de cabo de comprimento inicial ds, comprimento final $\mathrm{d} \overline{\mathrm{s}}$ e comprimento de referência (indeformado) $\mathrm{ds}_{\mathrm{o}}$.

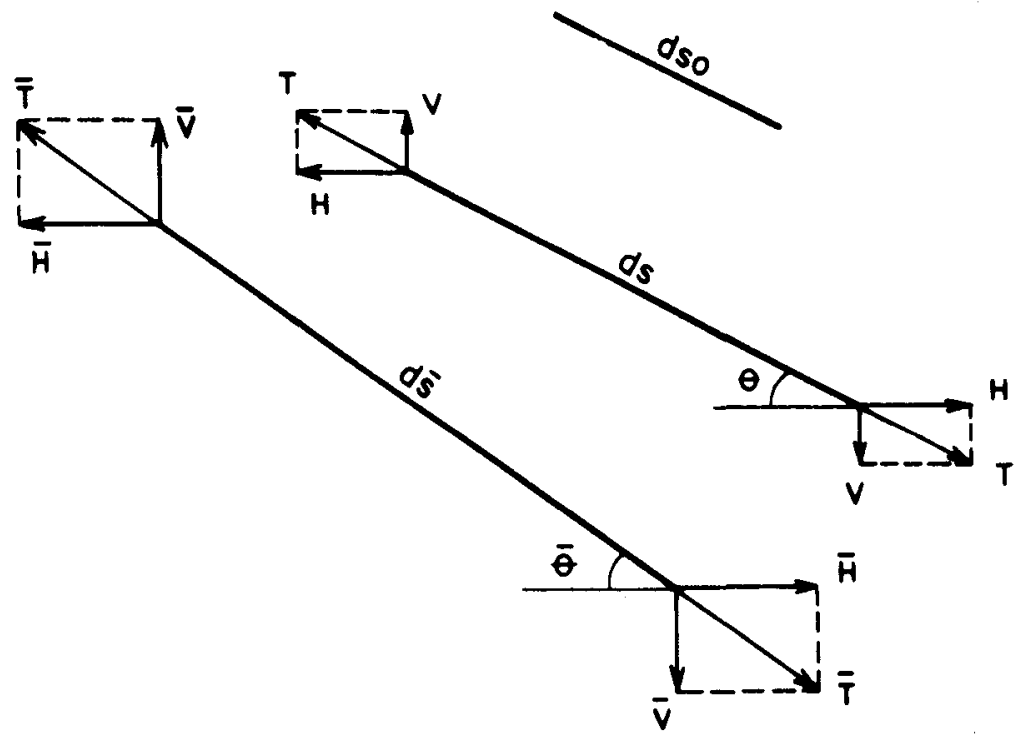

Figura 3.2.3.1 - Elementos de cabo nas posições indeformada, inicial e final. 
De acordo com a lei de Hooke, podem-se escrever da seguinte forma as equações que relacionam tensões e deformações:

$\sigma=\frac{T}{A}=E \frac{d s-d s_{0}}{d s_{o}}$

$\bar{\sigma}=\frac{\bar{T}}{A}=E \frac{d \bar{s}-d s_{o}}{d s_{o}}$

sendo A a área da seção transversal do cabo e E o seu módulo de elasticidade.

Combinando-se as equações (3.2.3.1), (3.2.3.2) e (3.2.2.3), e considerando-se a variação de temperatura, tem-se:

$\varepsilon=\frac{(\bar{T}-T)}{T+E A}+\alpha \Delta t$

onde $\alpha$ é o coeficiente linear de dilatação térmica do material da cabo e $\Delta$ t é a variação de temperatura.

\subsection{CONFIGURAÇÃO INICIAL DE EQUILÍBRIO}

Seja o cabo livremente suspenso esquematizado na figura 3.3.1:
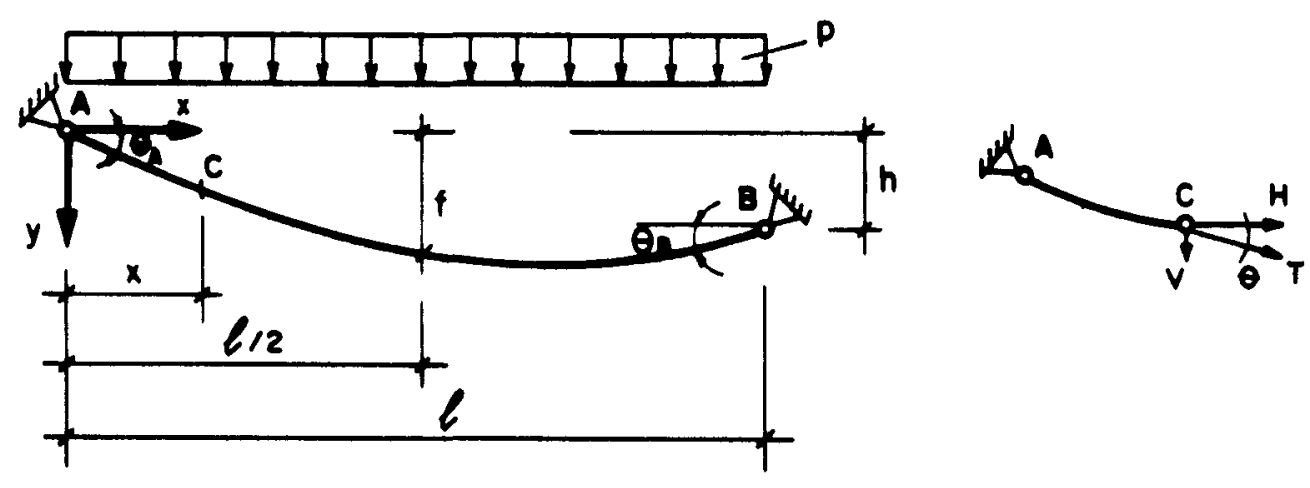

Figura 3.3.1 - Cabo na posição inicial de equilíbrio. 
Combinando-se as equações (3.2.1.1.1), (3.2.1.1.2) e (3.2.1.1.3) obtém-se como resultado a equação diferencial que caracteriza a configuração inicial de equilíbrio do cabo:

$y^{\prime \prime}=-\frac{p}{H}$

Integrando-se a equação (3.3.1), têm-se:

$y^{\prime}=-\frac{p}{H} x+a$

$y=-\frac{p}{2 H} x^{2}+a x+b$

Especificando-se as coordenadas $\mathrm{x}$ e $\mathrm{y}$ dos pontos $\mathrm{A}$ e $\mathrm{B}$, determinam-se as constantes de integração:

$\mathrm{b}=0$

$\mathrm{a}=\frac{\mathrm{p} \ell}{2 \mathrm{H}}+\frac{\mathrm{h}}{\ell}$

Introduzindo-se estas duas últimas equações em (3.3.2) e (3.3.3), determinamse as equações que caracterizam a curva do cabo:

$$
\begin{aligned}
& y^{\prime}=-\frac{p}{H} x+\left(\frac{p \ell}{2 H}+\frac{h}{\ell}\right) \\
& y=-\frac{p}{2 H} x^{2}+\left(\frac{p \ell}{2 H}+\frac{h}{\ell}\right) x
\end{aligned}
$$

A equação (3.3.6) permite determinar o ângulo que a tangente à curva do cabo faz com o eixo das abscissas e a (3.3.7) fornece a ordenada dos pontos do cabo em relação ao mesmo eixo.

A partir destas equações pode-se obter todos os parâmetros definidores da configuração de equilíbrio do cabo. Assim, determinam-se a força 
horizontal $\mathbf{H}$ - constante ao longo do cabo, a força de tração $\mathbf{T}$, o comprimento do cabo $S$ e, também, os ângulos $\theta_{A}$ e $\theta_{B}$

A força horizontal $\mathbf{H}$ pode ser obtida ou em função do ângulo $\theta_{\mathrm{A}}$ ou em função da flecha do cabo, figura 3.3.1.

Quando se conhece o ângulo $\theta_{\mathrm{A}}$, da equação (3.3.6), com $x=0$ e $y^{\prime}=\operatorname{tg} \theta_{\mathrm{A}}$, obtémse

$$
\mathrm{H}=\frac{\mathrm{p} \ell^{2}}{2\left(\ell \operatorname{tg} \theta_{\mathrm{A}}-\mathrm{h}\right)}
$$

Quando a flecha do cabo é conhecida, de (3.3.7), com $x=\ell / 2$ e y $=f$, tira-se:

$$
\mathrm{H}=\frac{\mathrm{p} \ell^{2}}{4(2 \mathrm{f}-\mathrm{h})}
$$

A força de tração $\mathrm{T}$, variável ao longo do cabo, figura 3.3.1, pode ser calculada por

$$
\mathrm{T}=\frac{\mathrm{H}}{\cos \theta}=\mathrm{H} \sqrt{1+\mathrm{y}^{\prime 2}}
$$

Tendo em vista a expressão de y', dada por (3.3.6), obtém-se:

$$
\mathrm{T}=\mathrm{H} \sqrt{1+\left[-\frac{\mathrm{p}}{\mathrm{H}} \mathrm{x}+\left(\frac{\mathrm{p} \ell}{2 \mathrm{H}}+\frac{\mathrm{h}}{\ell}\right)\right]^{2}}
$$

As forças de tração $T_{A}$ e $T_{B}$ que surgem nos pontos de suspensão podem ser calculadas por (3.3.11) fazendo-se $\mathrm{x}=0 \mathrm{e} \mathrm{x}=\ell$ respectivamente.

O comprimento do cabo é dado pela expressão resultante da integração, ao longo do vão, da expressão de ds

$$
\mathrm{s}=\int_{0}^{l} \mathrm{ds}=\int_{0}^{l} \sqrt{(\mathrm{dx})^{2}+(\mathrm{dy})^{2}}=\int_{0}^{l} \sqrt{1+\mathrm{y}^{\prime 2}} \mathrm{dx}
$$

donde, com boa aproximação, obtém-se 
$\left.\mathrm{s} \cong \int_{0} \int_{0} 1+\frac{\mathrm{y}^{\prime 2}}{2}\right) \mathrm{dx}=\ell\left[1+\left(\frac{\mathrm{p}}{\mathrm{H}}\right)^{2} \frac{\ell^{2}}{24}+\frac{\mathrm{h}^{2}}{2 \ell^{2}}\right]$

\subsection{CONFIGURAÇÃO FINAL DE EQULÍBRIO}

Estando definida a posição de equilibrio do cabo para o carregamento inicial $\mathrm{p}$, pode-se estudar a deformação do cabo sob o carregamento final $\overline{\mathrm{p}}=\mathrm{p}+\Delta \mathrm{p}$.
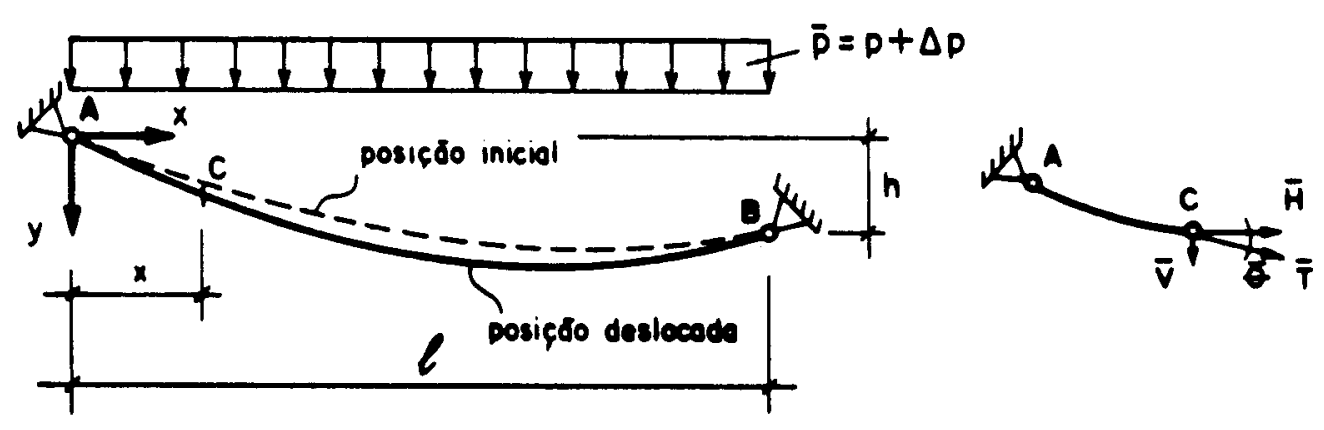

Figura 3.4.1 - Cabo na posição final de equilíbrio.

Combinando-se (3.2.1.2.1), (3.2.1.2.2) e (3.2.1.2.3), obtém-se:

$y^{\prime}+v^{\prime}=\left[-\frac{\bar{p}}{\bar{H}} \int\left(1+u^{\prime}\right) d x+k_{1}\right]\left(1+u^{\prime}\right)$

Fazendo-se a aproximação $\left(1+u^{\prime}\right) \cong 1$ na equação (3.4.1), tem-se:

$v^{\prime}=-\frac{\bar{p}}{\overline{\mathbf{H}}} x+k_{1}-y^{\prime}$

Introduzindo-se (3.3.2) em (3.4.2) e integrando-se a equação resultante, obtém-se:

$v=\left(\frac{p}{H}-\frac{\bar{p}}{\bar{H}}\right) \frac{x^{2}}{2}+\left(k_{1}-a\right) x+k_{2}$ 
Fazendo-se $\mathrm{v}=\mathrm{v}_{\mathrm{A}}$ para $\mathrm{x}=0 \mathrm{e} \mathrm{v}=\mathrm{v}_{\mathrm{B}}$ para $\mathrm{x}=\ell$ na equação anterior, calculam-se as constantes de integração $k_{1}$ e $k_{2}$ e portanto:

$$
\begin{aligned}
& v^{\prime}=\left(\frac{p}{H}-\frac{\bar{p}}{\bar{H}}\right) x+\left[\frac{v_{B}-v_{A}}{\ell}-\left(\frac{p}{H}-\frac{\bar{p}}{\bar{H}}\right) \frac{\ell}{2}\right] \\
& v=\left(\frac{p}{H}-\frac{\bar{p}}{\bar{H}}\right) \frac{x^{2}}{2}+\left[\frac{v_{B}-v_{A}}{\ell}-\left(\frac{p}{H}-\frac{\bar{p}}{\bar{H}}\right) \frac{\ell}{2}\right] x+v_{A}
\end{aligned}
$$

Considerando-se as equações (3.2.2.4) e (3.2.3.3), e observando - figuras (3.2.1.1.1), (3.2.1.2.1) e (3.2.3.1) - que

$$
\begin{aligned}
& \cos \theta=\frac{1}{\sqrt{1+\mathrm{y}^{\prime 2}}} \\
& \cos \bar{\theta}=\frac{1}{\sqrt{1+\left(\frac{\mathrm{y}^{\prime}+\mathrm{v}^{\prime}}{1+\mathrm{u}^{\prime}}\right)^{2}}} \\
& \mathrm{~T}=\mathrm{H} \sqrt{1+\mathrm{y}^{\prime 2}} \\
& \overline{\mathrm{T}}=\overline{\mathrm{H}} \sqrt{1+\left(\frac{\mathrm{y}^{\prime}+\mathrm{v}^{\prime}}{1+\mathrm{u}^{\prime}}\right)^{2}}
\end{aligned}
$$

obtém-se

$$
\sqrt{1+\frac{2\left(u^{\prime}+y^{\prime} v^{\prime}\right)+u^{\prime 2}+v^{\prime 2}}{1+y^{\prime 2}}}-1=\frac{\bar{H} \sqrt{1+\left(\frac{y^{\prime}+v^{\prime}}{1+u^{\prime}}\right)^{2}}-H \sqrt{1+y^{\prime 2}}}{E A+H \sqrt{1+y^{\prime 2}}}+\alpha \Delta t
$$

Fazendo-se, na equação (3.4.10), as aproximações

$$
\left(1+u^{\prime}\right) \cong 1
$$

$$
\sqrt{1+\frac{2\left(u^{\prime}+y^{\prime} v^{\prime}\right)+u^{\prime 2}+v^{\prime 2}}{1+y^{\prime 2}}} \cong 1+\frac{2\left(u^{\prime}+y^{\prime} v^{\prime}\right)+u^{\prime 2}+v^{\prime 2}}{2\left(1+y^{\prime 2}\right)}
$$


$\sqrt{1+\left(y^{\prime}+v^{\prime}\right)^{2}} \cong 1+\sqrt{1+y^{\prime 2}}+\frac{v^{\prime 2}}{2}+v^{\prime} y^{\prime}$

obtém-se

$u^{\prime}=\left(\frac{\bar{H}}{E A+H \sqrt{1+y^{\prime 2}}}-1\right)\left(y^{\prime} v^{\prime}+\frac{v^{\prime 2}}{2}\right)+\bar{H} \frac{\left(y^{\prime 3} v^{\prime}+\frac{y^{\prime 2} v^{\prime 2}}{2}\right)}{E A+H \sqrt{1+y^{\prime 2}}}+(\bar{H}-H) f_{1}(x)+f_{2}(x)$

Nesta equação, $f_{1}(x)$ e $f_{2}(x)$ se expressam por

$f_{1}(x)=\frac{\left(1+y^{\prime 2}\right)^{3 / 2}}{E A+H \sqrt{1+y^{\prime 2}}}$

$f_{2}(x)=\alpha \Delta t\left(1+y^{\prime 2}\right)$

Fazendo-se, ainda, a aproximação

$\mathrm{EA}+\mathrm{H} \sqrt{1+\mathrm{y}^{\prime 2}} \cong \mathrm{EA}+\mathrm{H}$

nos termos da equação (3.4.14) que após esta aproximação ficam na forma polinomial e integrando-se a equação resultante, obtém-se:

$$
\begin{aligned}
u= & \left(\frac{\bar{H}}{E A+H}-1\right) \int y^{\prime} v^{\prime} d x+\frac{1}{2}\left(\frac{\bar{H}}{E A+H}-1\right) \int v^{\prime 2} d x+\frac{\bar{H}}{E A+H} \int y^{\prime 3} v^{\prime} d x+ \\
& +\frac{\bar{H}}{2(E A+H)} \int y^{\prime 2} v^{\prime 2} d x+(\bar{H}-H) \int f_{1}(x) d x+\int f_{2}(x) d x+k_{3}
\end{aligned}
$$

Com a resolução das integrais da equação anterior e imposição das condições de contorno, $\mathrm{u}=\mathrm{u}_{\mathrm{A}}$ para $\mathrm{x}=0$ e $\mathrm{u}=\mathrm{u}_{\mathrm{B}}$ para $\mathrm{x}=\ell$, obtêm-se:

$$
\mathrm{k}_{3}=\mathrm{u}_{\mathrm{A}}
$$


$\alpha_{1} \overline{\mathrm{H}}^{3}+\beta_{1} \overline{\mathrm{H}}^{2}+\gamma_{1} \overline{\mathrm{H}}+\delta_{1}=0$

Os coeficientes da equação cúbica (3.4.20) são números reais que dependem da posição inicial de equilíbrio do cabo, do seu módulo de elasticidade, da área de sua seção transversal, do seu carregamento final e dos deslocamentos de seus apoios. $\mathrm{E}$ podem ser escritos da seguinte forma:

$$
\begin{aligned}
\alpha_{1}= & \frac{1}{E A+H}\left[A_{1}\left(\frac{p}{H}\right)+A_{2}+\left(\frac{p}{H}\right)^{2} B_{1}+B_{2}\right]+ \\
& +\left(\frac{p}{H}\right) C_{1}+C_{2}+\left(\frac{p}{H}\right)^{2} D_{1}+\left(\frac{p}{H}\right) D_{2}+D_{3}+E_{1} \\
\beta_{1}= & -\frac{A_{1} \bar{p}}{E A+H}-A_{1}\left(\frac{p}{H}\right)-A_{2}+\left(\frac{p}{H}\right) B_{1}\left[\frac{-2 \bar{p}}{E A+H}-\frac{p}{H}\right]-B_{2}+ \\
& -\bar{p} C_{1}-2\left(\frac{p}{H}\right) \bar{p} D_{1}-\bar{p} D_{2}-H E_{1}+F_{1}+\left(u_{A}-u_{B}\right) \\
\gamma_{1}= & A_{1} \bar{p}+\bar{p} B_{1}\left[\frac{\bar{p}}{E A+H}+2\left(\frac{p}{H}\right)\right]+\bar{p}^{2} D_{1} \\
\delta_{1}= & -\bar{p}^{2} B_{1}
\end{aligned}
$$

onde $A_{1}, A_{2}, B_{1}, B_{2}, C_{1}, C_{2}, D_{1}, D_{2}, D_{3}, E_{1}$ e $F_{1}$ são dados por

$$
\begin{aligned}
& \mathrm{A}_{1}=-\left(\frac{\mathrm{p}}{\mathrm{H}}\right) \frac{\ell^{3}}{12} \\
& \mathrm{~A}_{2}=\left(\frac{\mathrm{v}_{\mathrm{B}}-\mathrm{v}_{\mathrm{A}}}{\ell}\right)\left[-\frac{\mathrm{p}}{\mathrm{H}} \frac{\ell}{2}+\mathrm{a}\right] \ell
\end{aligned}
$$


$\mathrm{B}_{1}=\frac{\ell^{3}}{24}$

$\mathrm{B}_{2}=\frac{\ell}{2}\left(\frac{\mathrm{v}_{\mathrm{B}}-\mathrm{v}_{\mathrm{A}}}{\ell}\right)^{2}$

$C_{1}=\left[-3\left(\frac{p}{H}\right)^{3} \frac{\ell^{5}}{40}+a\left(\frac{p}{H}\right)^{2} \frac{\ell^{4}}{4}-a^{2}\left(\frac{p}{H}\right) \frac{\ell^{3}}{4}\right] /(E A+H)$

$\mathrm{C}_{2}=\left(\frac{\mathrm{v}_{\mathrm{B}}-\mathrm{v}_{\mathrm{A}}}{\ell}\right)\left[-\left(\frac{\mathrm{p}}{\mathrm{H}}\right)^{3} \frac{\ell^{4}}{4}+\mathrm{a}\left(\frac{\mathrm{p}}{\mathrm{H}}\right)^{2} \ell^{3}-3 \mathrm{a}^{2}\left(\frac{\mathrm{p}}{\mathrm{H}}\right) \frac{\ell^{2}}{2}+\mathrm{a}^{3} \ell\right] /(\mathrm{EA}+\mathrm{H})$

$\mathrm{D}_{1}=\left[\left(\frac{\mathrm{p}}{\mathrm{H}}\right)^{2} \frac{\ell^{5}}{30}-\mathrm{a}\left(\frac{\mathrm{p}}{\mathrm{H}}\right) \frac{\ell^{4}}{12}+\frac{\mathrm{a}^{2} \ell^{3}}{12}\right] / 2(\mathrm{EA}+\mathrm{H})$

$\mathrm{D}_{2}=\left[\left(\frac{\mathrm{p}}{\mathrm{H}}\right)^{2}\left(\frac{\mathrm{v}_{\mathrm{B}}-\mathrm{v}_{\mathrm{A}}}{\ell}\right) \frac{\ell^{4}}{6}-\mathrm{a}\left(\frac{\mathrm{p}}{\mathrm{H}}\right)\left(\frac{\mathrm{v}_{\mathrm{B}}-\mathrm{v}_{\mathrm{A}}}{\ell}\right) \frac{\ell^{3}}{3}\right] / 2(\mathrm{EA}+\mathrm{H})$

$\mathrm{D}_{3}=\left(\frac{\mathrm{v}_{\mathrm{B}}-\mathrm{v}_{\mathrm{A}}}{\ell}\right)^{2}\left[\left(\frac{\mathrm{p}}{\mathrm{H}}\right)^{2} \frac{\ell^{3}}{3}-\mathrm{a}\left(\frac{\mathrm{p}}{\mathrm{H}}\right) \ell^{2}+\mathrm{a}^{2} \ell\right] / 2(\mathrm{EA}+\mathrm{H})$

$E_{1}=\int_{0}^{\ell} f_{1}(x) d x$

$$
F_{1}=\alpha \Delta t\left[\left(\frac{p}{H}\right)^{2} \frac{\ell^{3}}{3}-a\left(\frac{p}{H}\right) \ell^{2}+\left(1+a^{2}\right) \ell\right]
$$

Calculada a raiz real positiva da equação, o esforço horizontal final no cabo, podem-se determinar todas as outras grandezas relativas à configuração final de equilíbrio do cabo. 


\subsection{CONSIDERAÇÕES SOBRE AS CONDIÇÕES DE CONTORNO}

Como se mencionou anteriormente, os coeficientes da equação (3.4.20) dependem da posição inicial de equilíbrio do cabo, da área de sua seção transversal, do seu módulo de elasticidade, do seu carregamento final e dos deslocamentos $u_{A}$, $\mathrm{u}_{\mathrm{B}}, \mathrm{v}_{\mathrm{A}}$ e $\mathrm{v}_{\mathrm{B}}$ de seus apoios.

Para o cálculo desses deslocamentos, mediante um certo carregamento final, necessita-se conhecer a rigidez da estrutura de sustentação e a ação atuante nesta estrutura, ou seja, é preciso conhecer também a configuração final de equilíbrio do cabo.

Utiliza-se, então, de um processo iterativo para a resolução da indeterminação - os deslocamentos dos apoios dependem da configuração final de equilíbrio do cabo e esta depende daqueles - que consiste do seguinte: arbitram-se os deslocamentos dos apoios, determina-se $\overline{\mathrm{H}}$ e calculam-se os novos deslocamentos; o processo iterativo termina quando a diferença entre os deslocamentos dos apoios de uma iteração para a seguinte está dentro de uma tolerância preestabelecida. 


\section{PROCESSO DOS DESLOCAMENTOS}

\subsection{INTRODUÇÃO}

Os esforços e deslocamentos dos cabos livremente suspensos são determinados discretizando-os em segmentos retilíneos ligados entre si por pontos nodais, onde se supõem aplicadas as ações. Utiliza-se matriz de rigidez tangente que relaciona para a estrutura acréscimos de carregamentos com acréscimos de deslocamentos. Admite-se regime elástico linear com pequenas deformações e a invariabilidade da área da seção transversal do cabo.

\subsection{SISTEMA DE COORDENADAS}

Os eixos $\mathrm{x}$ e $\mathrm{y}$ são os eixos de referência nos quais se escrevem as coordenadas do elemento e de toda a estrutura, figura 4.2.1.

Utiliza-se um referencial na configuração inicial segundo o qual são medidos os deslocamentos dos nós. 


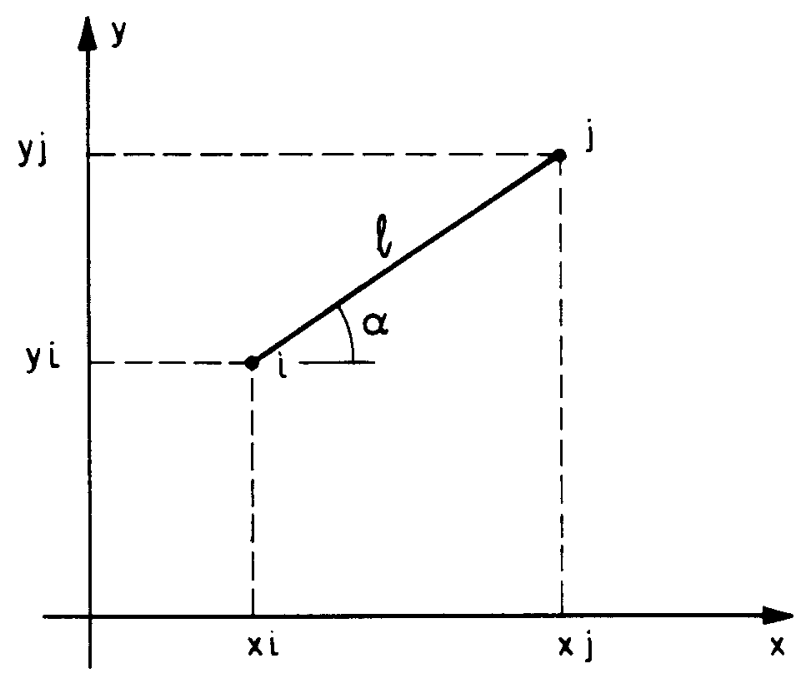

Figura 4.2.1 - Sistema de coordenadas.

\subsection{MATRIZ DE RIGIDEZ TANGENTE ( INSTANTÂNEA ) DO ELEMENTO}

Seja o elemento de cabo de eixo retilíneo, comprimento de referência $\left(\mathrm{s}_{0}\right)_{\mathrm{ij}}$, comprimento inicial $\ell_{\mathrm{ij}}$, comprimento genérico $(\ell+\mathrm{d} \ell) \mathrm{ij}$, comprimento final $(\ell+\Delta \ell)_{\mathrm{ij}}$ e extremidades $\mathrm{i}$ e $\mathrm{j}$ definidas pelos vetores-posição $\overrightarrow{\mathrm{r}}_{\mathrm{i}}$ e $\overrightarrow{\mathrm{r}}_{\mathrm{j}}$, figura 4.3.1.
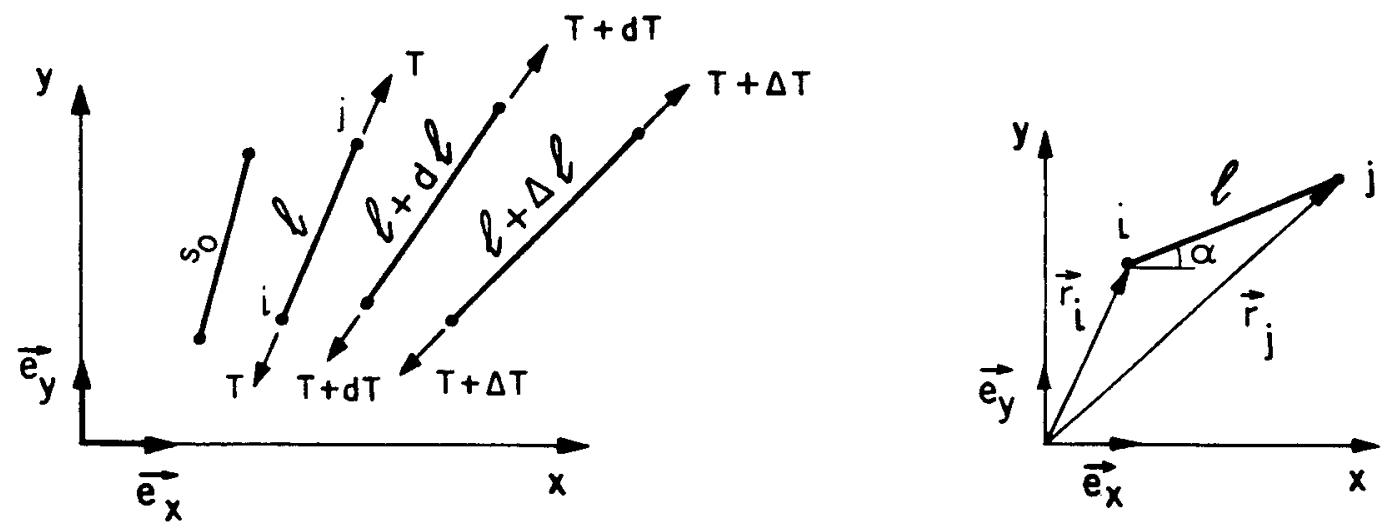

Figura 4.3.1 - Segmentos retilineos de cabo no sistema de coordenadas. 
$O$ módulo do vetor $\Delta \overrightarrow{\mathrm{r}}_{\mathrm{ij}}=\overrightarrow{\mathrm{r}}_{\mathrm{j}}-\overrightarrow{\mathrm{r}}_{\mathrm{i}}$ é $\ell_{\mathrm{ji}}=\ell_{\mathrm{ij}}$, então, o versor da direção $(\mathrm{i}, \mathrm{j})$ pode ser posto na forma:

$$
\overrightarrow{\mathrm{a}}_{\mathrm{ij}}=\frac{\overrightarrow{\mathrm{r}}_{\mathrm{j}}-\overrightarrow{\mathrm{r}}_{\mathrm{i}}}{\left|\overrightarrow{\mathrm{r}}_{\mathrm{j}}-\overrightarrow{\mathrm{r}}_{\mathrm{i}}\right|}=\frac{\Delta \overrightarrow{\mathrm{r}}_{\mathrm{ij}}}{\left|\Delta \overrightarrow{\mathrm{r}}_{\mathrm{ij}}\right|}=\frac{\Delta \overrightarrow{\mathrm{r}}_{\mathrm{ij}}}{\ell}=\left(\frac{\Delta \overrightarrow{\mathrm{r}}}{\ell}\right)_{\mathrm{ij}}
$$

Sendo $\overrightarrow{T i j}$ a força de tração atuante no elemento e $T_{i j}$ igual à $T_{j i}$ o seu módulo, e sabendo-se que

$$
\overrightarrow{\mathrm{T}}_{\mathrm{ij}}=\left(\overrightarrow{\mathrm{a}}_{\mathrm{ij}}\right) \mathrm{T}_{\mathrm{ij}}
$$

de (4.3.1) obtém-se:

$$
\overrightarrow{\mathrm{T}}_{\mathrm{ij}}=\left(\frac{\Delta \overrightarrow{\mathrm{r}}}{\ell}\right)_{\mathrm{ij}} \mathrm{T}_{\mathrm{ij}}
$$

Diferenciando-se a equação (4.3.3) — função de $\Delta \overrightarrow{\mathrm{r}}, \ell$ e T — resulta:

$$
\mathrm{d} \overrightarrow{\mathrm{T}}=\frac{\mathrm{T}}{\ell} \mathrm{d} \Delta \overrightarrow{\mathrm{r}}+\frac{\Delta \overrightarrow{\mathrm{r}}}{\ell}\left[\mathrm{dT}-\left(\frac{\mathrm{Td} \ell}{\ell}\right)\right]
$$

Admitindo-se a lei de Hooke, têm-se para o elemento de cabo em questão:

$$
\begin{aligned}
& \frac{T}{A}=E \frac{\left(\ell-s_{0}\right)}{s_{0}} \\
& \frac{(T+d T)}{A}=E\left[\frac{(\ell+d \ell)-s_{0}}{s_{0}}\right]
\end{aligned}
$$

sendo A a área da seção transversal do cabo e E o seu módulo de elasticidade. 
Da equação (4.3.5) podem-se escrever

$$
\begin{aligned}
& s_{o}=\frac{\ell E A}{E A+T} \\
& \ell=\frac{s_{0}}{E A}(E A+T)
\end{aligned}
$$

Diferenciando-se a equação (4.3.8), obtém-se:

$$
\mathrm{d} \ell=\frac{\mathrm{s}_{\mathrm{O}}}{\mathrm{EA}}(\mathrm{EA}+\mathrm{dT})
$$

Introduzindo-se (4.3.9) em (4.3.6) e diferenciando-se a equação resultante, obtém-se:

$$
\mathrm{d} \ell=\frac{\mathrm{dTs}_{\mathrm{o}}}{\mathrm{EA}}
$$

Introduzindo-se (4.3.7) em (4.3.10), tem-se:

$$
\mathrm{dT}=(\mathrm{EA}+\mathrm{T}) \frac{\mathrm{d} \ell}{\ell}
$$

Combinando-se as equações (4.3.11) e (4.3.4) resulta a equação vetorial que dá origem a matriz de rigidez tangente do elemento de cabo:

$$
\mathrm{d} \overrightarrow{\mathrm{T}}=\frac{\mathrm{T}}{\ell} \mathrm{d}(\Delta \overrightarrow{\mathrm{r}})+\left(\frac{\mathrm{EA}}{\ell} \frac{\mathrm{d} \ell}{\ell}\right) \Delta \overrightarrow{\mathrm{r}}
$$

Com o auxílio da figura 4.3.1, escrevem-se:

$$
\overrightarrow{\mathrm{r}}_{\mathrm{i}}=\left(\mathrm{x}_{\mathrm{i}}\right) \overrightarrow{\mathrm{e}}_{\mathrm{x}}+\left(\mathrm{y}_{\mathrm{i}}\right) \overrightarrow{\mathrm{e}}_{\mathrm{y}}
$$


$\overrightarrow{\mathrm{r}}_{\mathrm{j}}=\left(\mathrm{x}_{\mathrm{j}}\right) \overrightarrow{\mathrm{e}}_{\mathrm{x}}+\left(\mathrm{y}_{\mathrm{j}}\right) \overrightarrow{\mathrm{e}}_{\mathrm{y}}$

$\left(\Delta \vec{r}_{i j}\right)=\Delta \vec{r}=\left(x_{j}-x_{i}\right) \vec{e}_{x}+\left(y_{j}-y_{i}\right) \vec{e}_{y}$

$\ell_{\mathrm{ij}}=\ell_{\mathrm{ji}}=\ell=\left[\left(\mathrm{x}_{\mathrm{j}}-\mathrm{x}_{\mathrm{i}}\right)^{2}+\left(\mathrm{y}_{\mathrm{j}}-\mathrm{y}_{\mathrm{i}}\right)^{2}\right]^{1 / 2}$

Sabendo-se que:

$\mathrm{d}\left(\Delta \overrightarrow{\mathrm{r}}_{\mathrm{ij}}\right)=\mathrm{d} \Delta \overrightarrow{\mathrm{r}}=\frac{\partial \Delta \overrightarrow{\mathrm{r}}}{\partial \mathrm{x}_{\mathrm{j}}} \mathrm{dx}_{\mathrm{j}}+\frac{\partial \Delta \overrightarrow{\mathrm{r}}}{\partial \mathrm{y}_{\mathrm{j}}} \mathrm{dy}_{\mathrm{j}}+\frac{\partial \Delta \overrightarrow{\mathrm{r}}}{\partial \mathrm{x}_{\mathrm{i}}} \mathrm{dx_{i }}+\frac{\partial \Delta \overrightarrow{\mathrm{r}}}{\partial \mathrm{y}_{\mathrm{i}}} \mathrm{dy}$

$\mathrm{d}\left(\ell_{\mathrm{ij}}\right)=\mathrm{d} \ell=\frac{\partial \ell}{\partial \mathrm{x}_{\mathrm{j}}} \mathrm{dx}_{\mathrm{j}}+\frac{\partial \ell}{\partial \mathrm{y}_{\mathrm{j}}} \mathrm{dy}_{\mathrm{j}}+\frac{\partial \ell}{\partial \mathrm{x}_{\mathrm{i}}} \mathrm{dx}_{\mathrm{i}}+\frac{\partial \ell}{\partial \mathrm{y}_{\mathrm{i}}} \mathrm{dy}_{\mathrm{i}}$

e que as bases $-\overrightarrow{\mathrm{e}}_{\mathrm{x}}$ e $\overrightarrow{\mathrm{e}}_{\mathrm{y}}$ - do espaço vetorial são constantes, têm-se os diferenciais totais de $\Delta \overrightarrow{\mathrm{r}}$ e $\ell$ :

$d \Delta \vec{r}=\left(d x_{j}-d x_{i}\right) \vec{e}_{x}+\left(d y_{j}-d y_{i}\right) \vec{e}_{y}$

$\mathrm{d} \ell=\left(\mathrm{x}_{\mathrm{j}}-\mathrm{x}_{\mathrm{i}}\right)\left(\mathrm{dx}_{\mathrm{j}}-\mathrm{dx} \mathrm{x}_{\mathrm{i}}\right)+\left(\mathrm{y}_{\mathrm{j}}-\mathrm{y}_{\mathrm{i}}\right)\left(\mathrm{dy_{j }}-\mathrm{dy_{ \textrm {i } }}\right)$

Escrevendo-se a equação (4.3.12) em função das coordenadas $x_{i}, y_{i}, x_{j}$ e $y_{j}$ e dos deslocamentos diferenciais $\mathrm{dx}_{\mathrm{i}}, \mathrm{dy}_{\mathrm{i}}, \mathrm{dx}_{\mathrm{j}}$ e $\mathrm{dy}_{\mathrm{j}}$, tem-se:

$$
\begin{aligned}
\mathrm{dT}= & \frac{\mathrm{T}}{\ell}\left[\left(d x_{j}-d x_{i}\right) \overrightarrow{\mathrm{e}}_{\mathrm{x}}+\left(d y_{j}-d y_{i}\right) \vec{e}_{y}\right]+ \\
& +\frac{E A}{\ell}\left[\frac{\left(x_{j}-x_{i}\right)\left(d x_{j}-d x_{i}\right)+\left(y_{j}-y_{i}\right)\left(d y_{j}-d y_{i}\right)}{\ell^{2}}\right]\left[\left(x_{j}-x_{i}\right) \vec{e}_{x}+\left(y_{j}-y_{i}\right) \vec{e}_{y}\right]
\end{aligned}
$$




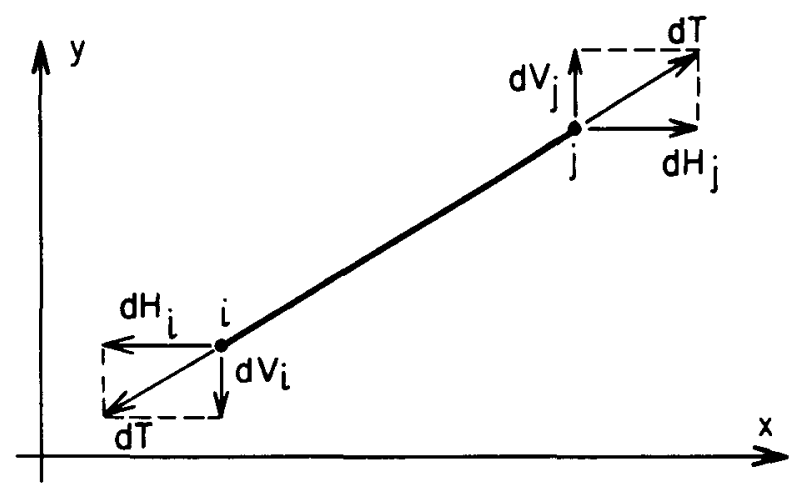

Figura 4.3.2 - Segmento de cabo e os esforços atuantes.

As forças $d H_{j}, d V_{j}, d H_{i} e d V_{i}$, componentes de $d T$, aplicadas às extremidades do elemento, figura 4.3.2, são dadas por:

$$
\begin{aligned}
d H_{j} & =d \vec{T} \cdot \vec{e}_{x}=\frac{T}{\ell}\left(d x_{j}-d x_{i}\right)+\frac{E A}{\ell}\left[\frac{\left(x_{j}-x_{i}\right)^{2}\left(d x_{j}-d x_{i}\right)}{\ell^{2}}+\right. \\
& \left.+\frac{\left(x_{j}+x_{i}\right)\left(y_{j}-y_{i}\right)\left(d y_{j}-d y_{i}\right)}{\ell^{2}}\right] \\
d V_{j} & =d \vec{T}_{\cdot} \cdot \vec{e}_{y}=\frac{T}{\ell}\left(d y_{j}-d y_{i}\right)+\frac{E A}{\ell}\left[\frac{\left(x_{j}-x_{i}\right)\left(y_{j}-y_{i}\right)\left(d x_{j}-d x_{i}\right)}{\ell^{2}}+\right. \\
& \left.+\frac{\left(y_{j}-y_{i}\right)^{2}\left(d y_{j}-d y_{i}\right)}{\ell^{2}}\right]
\end{aligned}
$$




$$
\begin{aligned}
d H_{i} & =d \vec{T}-\vec{e}_{x}=\frac{T}{\ell}\left(d x_{i}-d x_{j}\right)+\frac{E A}{\ell}\left[\frac{\left(x_{j}-x_{i}\right)^{2}\left(d x_{i}-d y_{j}\right)}{\ell^{2}}+\right. \\
& \left.+\frac{\left(x_{j}-x_{i}\right)\left(y_{j}-y_{i}\right)\left(d y_{i}-d y_{j}\right)}{\ell^{2}}\right] \\
d V_{i} & =d \vec{T}_{-}-\vec{e}_{y}=\frac{T}{\ell}\left(d y_{i}-d y_{j}\right)+\frac{E A}{\ell}\left[\frac{\left(x_{j}-x_{i}\right)\left(y_{j}-y_{i}\right)\left(d x_{i}-d x_{j}\right)}{\ell^{2}}+\right. \\
& \left.+\frac{\left(y_{j}-y_{i}\right)^{2}\left(d y_{i}-d y_{j}\right)}{\ell^{2}}\right]
\end{aligned}
$$

Da figura 4.3.1, pode-se escrever as seguintes relações geométricas:

$$
\begin{aligned}
& \cos \alpha=\frac{x_{j}-x_{i}}{\ell} \\
& \operatorname{sen} \alpha=\frac{y_{j}-y_{i}}{\ell}
\end{aligned}
$$

Combinando-se as equações (4.3.26), (4.3.27), (4.3.22), (4.3.23), (4.3.24) e (4.3.25), obtém-se:

$$
\left.\left\{\begin{array}{l}
d_{H_{i}} \\
d_{i} \\
d H_{j} \\
d V_{j}
\end{array}\right\}=\left[\left(\frac{T}{\ell}\right)\left[\begin{array}{cccc}
1 & 0 & -1 & 0 \\
0 & 1 & 0 & -1 \\
-1 & 0 & 1 & 0 \\
0 & -1 & 0 & 1
\end{array}\right]+\left(\frac{E A}{\ell}\right)\left[\begin{array}{cccc}
\cos ^{2} \alpha & \operatorname{sen} \alpha \cos \alpha & -\cos ^{2} \alpha & -\operatorname{sen} \alpha \cos \alpha \\
\operatorname{sen} \alpha \cos \alpha & \operatorname{sen}^{2} \alpha & -\operatorname{sen} \alpha \cos \alpha & -\operatorname{sen}^{2} \alpha \\
-\cos ^{2} \alpha & -\operatorname{sen} \alpha \cos \alpha & \cos \alpha & \operatorname{sen} \alpha \cos \alpha \\
-\operatorname{sen} \alpha \cos \alpha & -\operatorname{sen}^{2} \alpha & \operatorname{sen} \alpha \cos \alpha & \operatorname{sen}^{2} \alpha
\end{array}\right]\right\} \begin{array}{l}
\mathrm{dx}_{\mathrm{i}} \\
\mathrm{dy}_{\mathrm{i}} \\
\mathrm{dx}_{\mathrm{j}} \\
\mathrm{dy}_{\mathrm{j}}
\end{array}\right\}
$$


ou seja:

$$
\left\{\begin{array}{l}
d H_{i} \\
d V_{i} \\
d H_{j} \\
d V_{j}
\end{array}\right\}=\left[K_{T}\right]\left\{\begin{array}{l}
d x_{i} \\
d y_{i} \\
d x_{j} \\
d y_{j}
\end{array}\right\}=\left[K_{G}+K_{E}\right]\left\{\begin{array}{l}
d x_{i} \\
d y_{i} \\
d x_{j} \\
d y_{j}
\end{array}\right\}
$$

As deduções realizadas mostram a matriz tangente $\mathrm{K}_{\mathrm{T}}$ escrita em função da matriz geométrica $\mathrm{K}_{\mathrm{G}}$ e da matriz elástica linear $\mathrm{K}_{\mathrm{E}}$.

A matriz de rigidez da estrutura é determinada a partir das contribuições de todos os elementos.

\subsection{PROCESSO ITERATIVO DE RESOLUÇÃO}

No processo de resolução, numa iteração genérica monta-se a matriz de rigidez da estrutura, calculam-se os deslocamentos nodais gerados por forças desequilibradas, determinam-se as forças que surgem nas barras e verifica-se o equilíbrio dos nós da estrutura; na primeira iteração a matriz de rigidez é a da estrutura em sua configuração inicial e as forças desequilibradas são as ações aplicadas na estrutura; em cada iteração, seguinte à primeira, a matriz de rigidez e as forças desequilibradas são sempre as da iteração anterior; o processo chega ao fim quando se constata, de acordo com uma precisão previamente estabelecida, o equilíbrio de todos os nós da estrutura.

Portanto, a matriz de rigidez tangente pode ser interpretada como um "propagador" de deslocamentos ao longo do sistema estrutural. A partir desses deslocamentos determinam-se as deformações e, com aplicação da equação constitutiva do material, os esforços internos que podem ou não equilibrar as ações externas. Quando equilibram, o processo atinge a convergência. Quando não, há a necessidade de se corrigir deslocamentos e retomar todo o procedimento até que a convergência se dê. 
Quando com pequenas variações dos deslocamentos têm-se grandes variações nos esforços internos deve-se aplicar o carregamento em parcelas e para cada uma destas o processo iterativo descrito. 


\section{APLICAÇÃO NUMÉRICA}

\subsection{INTRODUÇÃO}

Considera-se como exemplo de aplicação numérica, com a finalidade de mostrar o emprego dos processos apresentados nos capítulos 3 e 4, o cabo livremente suspenso, figura 5.1 .1 , com $E=120 \times 10^{9} \mathrm{~N} / \mathrm{m}^{2}, A=403,22 \mathrm{~mm}^{2}, \mathrm{f}=6 \mathrm{~m}, \ell=60 \mathrm{~m}$, $\mathrm{h}=0$ e $\overline{\mathrm{p}}=5000 \mathrm{~N} / \mathrm{m}$.
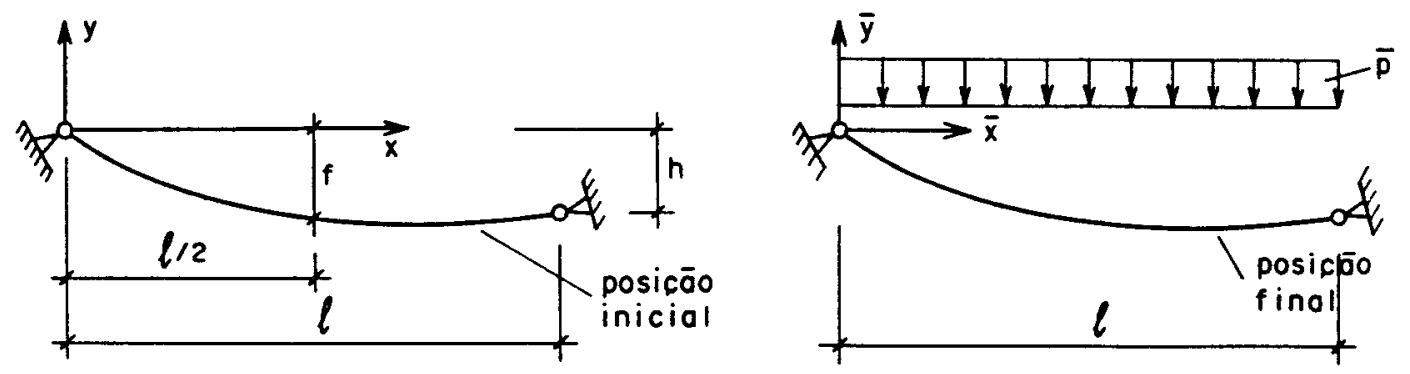

Figura 5.1.1 - Exemplo de aplicação numérica.

\subsection{CÁlCULO PELO PROCESSO ANALÍTICO}

Para definir as características da configuração inicial de equilíbrio é necessário conhecer o vão do cabo $\ell$, o desnivel dos pontos de suspensão $h$ e também a relação $\mathrm{P} / \mathrm{H}$. Conhecendo-se a ordenada y no meio do vão, a relação $\mathrm{P} / \mathrm{H}$ pode ser determinada a partir da equação (3.3.9), ou seja: 
$\left(\frac{p}{H}\right)=\frac{4(2 \cdot 6-0)}{60^{2}}=\frac{1}{75}$

A partir das equações (3.3.6) e (3.3.7), podem-se obter todos os parâmetros definidores da configuração inicial de equilíbrio do cabo. Assim, determinam-se a força horizontal $\mathbf{H}$, a força de tração $\mathbf{T}$, o comprimento do cabo s e, também, os ângulos $\theta_{\mathrm{A}}$ e $\theta_{\mathrm{B}}$ :

$y^{\prime}=-\frac{1}{75} x+\left(\frac{1}{75} \frac{60}{2}+\frac{0}{60}\right)=-\frac{1}{75} x+\frac{2}{5}$

$y=-\frac{1}{2} \frac{1}{75} x^{2}+\left(\frac{1}{75} \frac{60}{2}+\frac{0}{60}\right) x=-\frac{1}{150} x^{2}+\frac{2}{5} x$

$H=\frac{0.60^{2}}{4(2.6-0)}=0$

$\mathrm{T}=0 \sqrt{1+\left[-\frac{1}{75} \mathrm{x}+\left(\frac{1}{75} \frac{60}{2}+\frac{0}{60}\right)\right]^{2}}=0$

$\operatorname{tg} \theta_{\mathrm{A}}=-\frac{1}{75} \cdot 0+\frac{2}{5}=\frac{2}{5} \therefore \theta_{\mathrm{A}}=21,801^{\circ}$

$\theta_{\mathrm{B}}=-\theta_{\mathrm{A}}$

$s=60\left[1+\left(\frac{1}{75}\right)^{2} \frac{60^{2}}{24}+\frac{0^{2}}{2.60^{2}}\right]=61,600 \mathrm{~m}$

Estando definida a configuração inicial de equilíbrio do cabo, pode-se determinar a posição final de equilíbrio adicionando-se à posição inicial os deslocamentos u e $\mathrm{v}$ gerados pela ação de $5000 \mathrm{~N} / \mathrm{m}$, figura 5.2 .1 , através do procedimento descrito à seguir. 


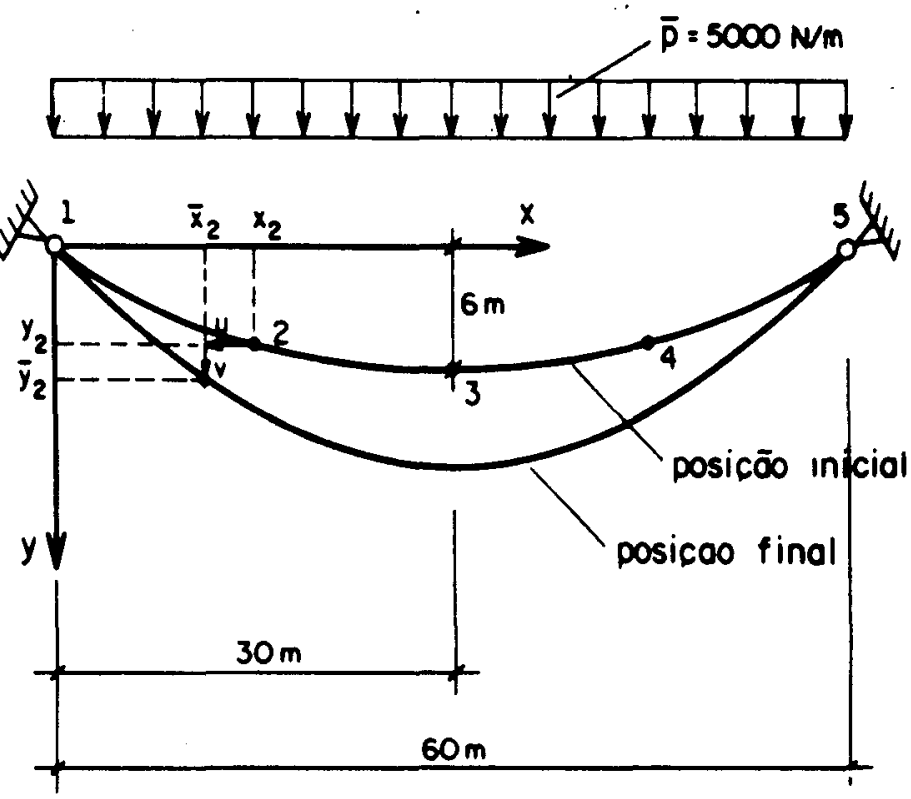

Figura 5.2.1 - Determinação da configuração final de equilíbrio.

Utilizando as equações de números (3.4.21) a (3.4.35), calculam-se os coeficientes da equação cúbica, cujos valores são os seguintes:

$\alpha_{1}=1,305315 \times 10^{-6}$

$\beta_{1}=1,600000$

$\gamma_{1}=5096,473389$

$\delta_{1}=-2,250000 \times 10^{11}$

Introduzindo-se os valores de $\alpha_{1}, \beta_{1}, \gamma_{1}$ e $\delta_{1}$ na equação cúbica (3.4.20), obtém-se $\overline{\mathrm{H}}=331454 \mathrm{~N}$

Através da equação (3.4.4), calcula-se:

$$
\begin{aligned}
& v^{\prime}=\left(\frac{1}{75}-\frac{5000}{331454}\right) x+\left[\frac{0-0}{60}-\left(\frac{1}{75}-\frac{5000}{331454}\right) \frac{60}{2}\right] \\
& v^{\prime}=\left(-1,752 \times 10^{-3}\right) x+\left(5,255 \times 10^{-2}\right)
\end{aligned}
$$


Determinam-se as expressões de v e u através das equações (3.4.5) e (3.4.18):

$$
\begin{aligned}
\mathrm{v}= & \left(\frac{1}{75}-\frac{5000}{331454}\right) \frac{\mathrm{x}^{2}}{2}+\left[\frac{0-0}{60}-\left(\frac{1}{75}-\frac{5000}{331454}\right) \frac{60}{2}\right] \mathrm{x}+0 \\
\mathrm{v}= & \left(-8,759 \times 10^{-4}\right) \mathrm{x}^{2}+\left(5,255 \times 10^{-2}\right) \mathrm{x} \\
\mathrm{u}= & \left(\frac{331454}{120 \times 10^{9} \times 403,22 \times 10^{-6}+0}-1\right) \int_{0}^{\mathrm{x}_{\mathrm{p}}} \mathrm{y}^{\prime} \mathrm{v}^{\prime} \mathrm{dx}+ \\
& +\frac{1}{2}\left(\frac{331454}{120 \times 10^{9} \mathrm{x} 403,22 \times 10^{-6}+0}-1\right) \int_{0}^{\mathrm{x}_{\mathrm{p}}} \mathrm{v}^{\prime 2} \mathrm{dx}+ \\
& +\left(\frac{331454}{120 \times 10^{9} \times 403,22 \times 10^{-6}+0}\right) \int_{0}^{\mathrm{x}_{\mathrm{p}}} \mathrm{y}^{\prime^{3}} \mathrm{v}^{\prime} \mathrm{dx}+ \\
& +\frac{1}{2}\left(\frac{331454}{120 \times 10^{9} \times 403,22 \times 10^{-6}+0}\right) \int_{0}^{x_{\mathrm{p}}} \mathrm{y}^{\prime 2} \mathrm{v}^{\prime 2} \mathrm{dx}+ \\
& +(331454-0) \int_{0}^{\mathrm{x}_{\mathrm{p}}} \frac{\left(1+\mathrm{y}^{\prime 2}\right)^{3 / 2}}{\mathrm{EA}+\mathrm{H} \sqrt{1+\mathrm{y}^{\prime 2}}} \mathrm{dx}+0+0
\end{aligned}
$$

onde $\mathrm{X}_{\mathrm{p}}$ é a abscissa do ponto a calcular o valor de $\mathrm{u}$.

Determina-se a força de tração no cabo através da equação (3.4.9), onde $\overline{\mathrm{H}}$, y'e v'são os determinados anteriormente e u'é dado pela equação (3.4.14).

Os resultados obtidos com os procedimentos descritos são mostrados na tabela 5.2.1.

Tabela 5.2.1- Resultados da análise do cabo pelo Processo Analítico.

\begin{tabular}{|c|c|c|r|c|c|c|c|c|}
\hline \multirow{3}{*}{ Ponto } & \multicolumn{3}{|c|}{ Posição inicial } & \multicolumn{4}{c|}{ Posição final } & \multicolumn{2}{|c|}{ Esforços finais } \\
\cline { 2 - 9 } & $\begin{array}{c}\mathrm{x} \\
(\mathrm{m})\end{array}$ & $\begin{array}{c}\mathrm{y} \\
(\mathrm{m})\end{array}$ & $\begin{array}{c}\mathrm{u} \\
(\mathrm{m})\end{array}$ & $\begin{array}{c}\mathrm{v} \\
(\mathrm{m})\end{array}$ & $\begin{array}{c}\overline{\mathrm{x}}=\mathrm{x}+\mathrm{u} \\
(\mathrm{m})\end{array}$ & $\begin{array}{c}\overline{\mathrm{y}}=\mathrm{y}+\mathrm{v} \\
(\mathrm{m})\end{array}$ & $\begin{array}{c}\overline{\mathrm{T}} \\
(\mathrm{N})\end{array}$ & $\begin{array}{c}\overline{\mathrm{H}} \\
(\mathrm{N})\end{array}$ \\
\hline 1 & 0,000 & 0,000 & 0,000 & 0,000 & 0,000 & 0,000 & 364677 & \\
2 & 15,000 & 4,500 & $-0,077$ & 0,591 & 14,923 & 5,091 & 339805 & \\
3 & 30,000 & 6,000 & 0,000 & 0,788 & 30,000 & 6,788 & 331454 & 331454 \\
4 & 45,000 & 4,500 & 0,077 & 0,591 & 45,077 & 5,091 & 339805 & \\
5 & 60,000 & 0,000 & 0,000 & 0,000 & 60,000 & 0,000 & 364677 & \\
\hline
\end{tabular}




\subsection{CÁLCULO PELO PROCESSO DOS DESLOCAMENTOS}

Os dados necessários ao cálculo do cabo pelo processo dos deslocamentos são os da configuração inicial de equilíbrio, a ação final e as características fisicas do material do cabo.

A posição inicial de equilibrio do cabo pode ser determinada através do Processo Analítico. $O$ cabo nesta posição inicial é dividido em segmentos retilíneos interconectados por pontos nodais onde são aplicadas as ações equivalentes, figura 5.3.1. O valor da ação equivalente $\overline{\mathrm{P}}$ é dado por

$\overline{\mathrm{P}}=\overline{\mathrm{p}} \ell /(\mathrm{n}-1)$

sendo $\mathrm{n}$ o número de pontos nodais.

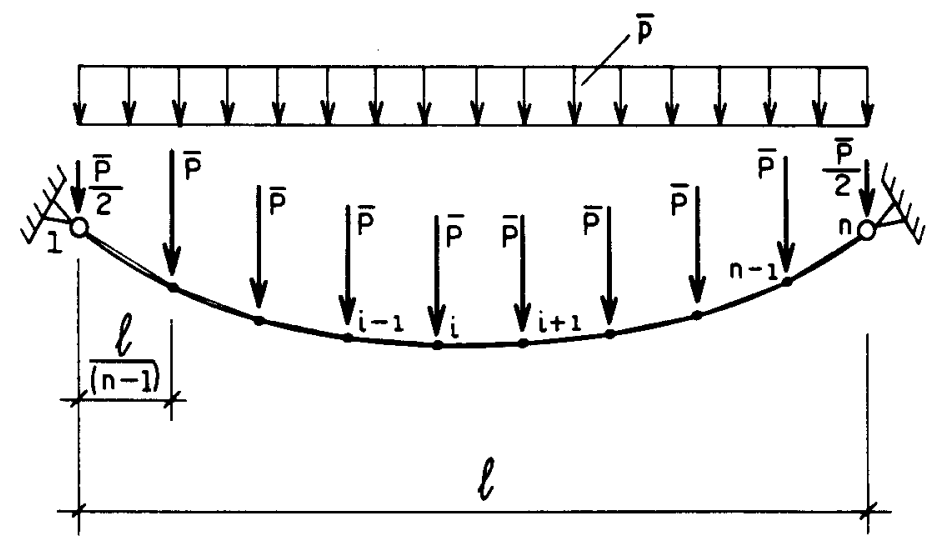

Figura 5.3.1 - Determinação do carregamento equivalente.

Encontra-se a configuração final de equilíbrio através de processo iterativo que faz a verificação das condições de equilibrio de todos os nós do cabo na posição deslocada.

Para a verificação do número de segmentos suficiente para descrever o comportamento do cabo, compararam-se os resultados com a variação do número de segmentos de 10 em 10 até 80 . Os resultados finais são os mostrados na tabela 5.3.1. 
Tabela 5.3.2 - Resultados da análise do cabo pelo Processo dos Deslocamentos.

\begin{tabular}{|c|c|c|c|c|c|c|}
\hline \multirow{2}{*}{ Nó } & \multicolumn{2}{|c|}{ Configuração inicial } & \multicolumn{2}{c|}{ Configuração final } & \multicolumn{2}{c|}{ Esforços finais } \\
\cline { 2 - 7 } & $\begin{array}{c}\mathbf{x} \\
(\mathrm{m})\end{array}$ & $\begin{array}{c}\mathrm{y} \\
(\mathrm{m})\end{array}$ & $\begin{array}{c}\overline{\mathbf{x}} \\
(\mathrm{m})\end{array}$ & $\begin{array}{c}\overline{\mathrm{y}} \\
(\mathrm{m})\end{array}$ & $\begin{array}{c}\overline{\mathrm{T}} \\
(\mathrm{N})\end{array}$ & $\begin{array}{c}\overline{\mathbf{H}} \\
(\mathrm{N})\end{array}$ \\
\hline 1 & 0,000 & 0,000 & 0,000 & 0,000 & 361593 & \\
21 & 15,000 & $-4,500$ & 14,925 & $-5,086$ & 338285 & \\
41 & 30,000 & $-6,000$ & 30,000 & $-6,799$ & 329866 & 329866 \\
61 & 45,000 & $-4,500$ & 45,075 & $-5,086$ & 338285 & \\
81 & 60,000 & 0,000 & 60,000 & 0,000 & 361593 & \\
\hline
\end{tabular}




\section{VERIFICAÇÃO DE RESULTADOS MEDIANTE EXPERIMENTAÇÃO NUMÉRICA}

\subsection{INTRODUÇÃo}

Tem-se como principal finalidade desta experimentação numérica a verificação das aproximações feitas no Processo Analítico através da comparação dos resultados deste processo com os obtidos pelo Processo dos Deslocamentos.

Faz-se também, a título ilustrativo, a comparação entre as expressões sem aproximações com as aproximadas empregadas no Processo Analítico.

Iniciando a experimentação numérica, verifica-se a precisão do Processo dos Deslocamentos para certificar-se da sua confiabilidade na verificação do Processo Analitico.

\subsection{CARACTERÍSTICAS DOS CABOS ESTUDADOS}

Descreve-se, a seguir, como foram determinadas as características mecânicas e geométricas dos cabos aqui empregados.

Como, sob o ponto de vista econômico, recomenda-se para as coberturas pênseis não-protendidas vão em torno de $40 \mathrm{a} 80 \mathrm{~m}$, espaçamento entre os cabos de 2 a $4 \mathrm{~m}$ e relação flecha/vão entre $1 / 10$ e $1 / 20$, para os cabos estudados especificaram-se vãos de 40,60 e $80 \mathrm{~m}$; espaçamento entre os cabos de 2 e $4 \mathrm{~m}$, e relação flecha/vão de 1/10 e 1/20.

As ações permanentes atuantes nas coberturas pênseis não-protendidas são, quase sempre, o peso próprio dos sistemas de vedação e estrutural e de equipamentos em geral. As ações variáveis são, normalmente, sobrecargas introduzidas na montagem e nas manutenções ao longo da vida útil das estruturas, efeitos do vento, acúmulos de águas pluviais e variações de temperatura. Assim sendo, considerou-se 
para os cabos estudados as ações de $2500,5000,7500$ e $10000 \mathrm{~N} / \mathrm{m}$, distribuídas ao longo dos vãos dos cabos.

Adotou-se, para cada caso, o diâmetro comercial determinado com o seguinte procedimento:

Predimensionou -se uma força de tração máxima no cabo, Tpd, igual a $80 \%$ da força horizontal resultante da aplicação do carregamento total no cabo, considerado inextensível, ou seja,

$\operatorname{Tpd}=80 \% \overline{\mathrm{H}}=80 \% \frac{\overline{\mathrm{p}} \ell^{2}}{4(2 \mathrm{f}-\mathrm{h})}$

Admitiu-se cabos com (AACI) - classificação 6x37 com tensão de ruptura mínima de $200 \mathrm{kgf} / \mathrm{mm}^{2}$. Adotando como coeficiente de segurança o fator 3 - indicado pelo fabricante dos cabos - encontrou-se como tensão admissível o valor de $66,67 \mathrm{Kgf} / \mathrm{mm}^{2}$. Dividindo o valor de Tpd por esta tensão admissível encontrou-se a área metálica mínima do cabo. Com as tabelas dos fabricantes, Apênces A.1 e A.3, chegou-se ao diâmetro comercial do cabo e à área metálica do cabo.

Nos cabos estudados adotou-se módulo de elasticidade igual a $120 \times 10^{9} \mathrm{~N} / \mathrm{m}^{2}$, de acordo com o Apêndice A.2.

\subsection{VERIFICAÇÃO DOS RESULTADOS DO PROCESSO DOS DESLOCAMENTOS}

Como os resultados da análise de cabos livremente suspenso submetidos a carregamentos distribuídos dependem do número de segmentos empregados, necessita-se saber se este número é suficiente para se obter bons resultados.

Mostra-se nas tabelas D.2, D.3 e D.4 (Apêndice D) exemplos da comparação realizada entre os resultados do Processo dos Deslocamentos com o aumento do número de segmentos empregados.

Constata-se que, aumentando o número de segmentos, os resultados do Processo dos Deslocamentos tendem a um valor que independe deste aumento. Por exemplo, na tabela D.2, o esforço $\overline{\mathrm{H}}$ para 10 segmentos é igual a $219024 \mathrm{~N}$, para 40 segmentos é igual $218975 \mathrm{~N}$ e para um número maior ou igual a 70 segmentos é igual a $218973 \mathrm{~N}$. 
Verifica-se que o número de segmentos suficiente é proporcional ao número do vão do cabo.

\subsection{VERIFICAÇÃO DOS RESULTADOS DO PROCESSO ANALÍTICO}

$\mathrm{Na}$ determinação das equações do Processo Analítico foram feitas as seguintes aproximações:

$$
\begin{aligned}
& 1+u^{\prime} \cong 1 \\
& E A+H \sqrt{1+y^{\prime 2}} \cong E A+H \\
& \sqrt{1+\left(y^{\prime}+v^{\prime}\right)^{2}} \cong \sqrt{1+y^{\prime 2}}+\frac{v^{\prime 2}}{2}+v^{\prime} y^{\prime} \\
& \sqrt{1+\frac{2\left(u^{\prime}+y^{\prime} v^{\prime}\right)+u^{\prime 2}+v^{\prime 2}}{1+y^{\prime 2}}} \cong 1+\frac{2\left(u^{\prime}+y^{\prime} v^{\prime}\right)+u^{\prime 2}+v^{\prime 2}}{2\left(1+y^{\prime 2}\right)}
\end{aligned}
$$

$\mathrm{Na}$ primeira aproximação, admitiu-se que u' é desprezivel na presença da unidade; na segunda admitiu-se que $\mathrm{y}^{\prime 2}$ é desprezível na presença da unidade e nas duas últimas admitiu-se que os dois primeiros termos da série são suficientes para a resolução da raiz quadrada.

Estudos feitos até agora empregaram valores do próprio Processo Analítico para verificar as suas aproximações. Questiona-se a confiabilidade de valores calculados a partir de hipóteses a serem comprovadas por estes mesmos valores. Fazse aqui, então, a verificação das aproximações empregadas no Processo Analítico através da comparação dos resultados deste processo com os do Processo dos Deslocamentos. Depois, faz-se a comparação entre os termos - originais e aproximados - destas quatro expressões empregadas.

Mostra-se na tabela D.5 (Apêndice D) comparação entre os resultados do Processo Analítico com os do Processo dos Deslocamentos quando se reduz apenas a área metálica do cabo, admitindo-se, porém, a linearidade física. Nesta tabela, verifica-se que, por exemplo, o erro que se comete quando se emprega o Processo Analítico em relação ao Processo dos Deslocamentos no cálculo da ordenada $\overline{\mathrm{y}}$ máxima do cabo $\mathrm{A} 7\left(\mathrm{~A}=1612,90 \mathrm{~mm}^{2}\right)$ é de - $0,10 \%$; e que para o cabo $\mathrm{A} 3$ 
$\left(\mathrm{A}=145,16 \mathrm{~mm}^{2}\right.$ - área mais de 10 vezes menor que a do cabo $\left.\mathrm{A} 7\right)$ esse erro é de $0,42 \%$.

Mostra-se nas tabelas D.6, D.7 e D.8 (Apêndice D) exemplos da comparação entre os resultados do Processo Analítico com os do Processo dos Deslocamentos quando se considera as variações usuais dos parâmetros dos cabos ( relação flecha/vão, vão, área metálica e carregamento ), variando-se um parâmetro por vez para certificar-se da sua influência nos resultados. Nestas tabelas verifica-se, por exemplo, que para os cabos B4, B10, B16 e B22 (predimensionados com um esforço de tração maior que o atuante) o erro que se comete quando se emprega o Processo Analítico em relação ao Processo dos Deslocamentos no cálculo de $\overline{\mathrm{H}}$ é de $0,3 \%$, e para os cabos B1, B7, B13 e B19 (predimensionados com um esforço de tração menor que o atuante - cabos mais deformáveis ) esse erro é de 0,4\%.

Mostra-se na tabela D.9 exemplos da comparação entre os resultados do Processo Analítico com os do Processo dos Deslocamentos quando se varia o parâmetro desnivel dos apoios.

Verifica-se, com os exemplos que comparam os resultados dos dois processos de cálculo, que o erro que se comete quando se emprega o Processo Analítico em relação ao Processo dos Deslocamentos depende apenas de quanto deformáveis são os cabos, sendo pequeno para pequenas deformações, aumentando com o aumento destas.

Mostra-se, ainda, na tabela D.10 exemplos da comparação entre as expressões originais e aproximadas empregadas no Processo Analítico. Verifica-se, como esperado, que as variações entre os termos destas expressões são maiores quanto maiores são as deformações dos cabos. 


\section{CONCLUSÃO}

Coberturas pênseis têm sido amplamente empregadas em todo o mundo para cobrir grandes áreas livres, como exemplifica a relação de obras mostrada no capítulo 1. Ainda assim, no Brasil, as coberturas suspensas são poucas vezes consideradas como uma real opção de projeto, o que torna importante a sua divulgação e estudo no país para alterar esta desagradável situação.

Este trabalho avança neste sentido, realizando:

- o desenvolvimento da formulação do Processo Analítico para análise de cabos livremente suspensos submetidos a carregamento uniformemente distribuído em todo o seu vão, a variações de temperatura e a deslocamentos dos seus apoios.

- a apresentação do Processo dos Deslocamentos para análise de sistemas estruturais de cabos de aço, em particular para os cabos livremente suspensos.

- da verificação da precisão dos resultados obtidos com o Processo dos Deslocamentos.

- da verificação das aproximações adotadas no Processo Analítico.

No Processo Analítico o estudo dos cabos livremente suspensos é realizado a partir de equações diferenciais em cujas resoluções são feitas algumas aproximações.

No Processo dos Deslocamentos a análise estática dos cabos é realizada discretizando-os em segmentos retilíneos ligados entre si por pontos nodais onde se supõem aplicadas as ações distribuídas. Determina-se o comportamento do cabo a partir da contribuição de todos os segmentos, estando a precisão do processo ligada ao número de segmentos empregados.

A verificação do número de segmentos suficiente para o cálculo do cabo pelo Processo dos Deslocamentos foi feita através da comparação dos resultados obtidos 
com a variação do número de segmentos empregados. Admitiu-se que o número de segmentos é suficiente quando o seu aumento não mais interfere nos resultados.

Na verificação das aproximações realizadas no Processo Analítico admitiu-se que estas poderiam ser feitas se os valores obtidos para $\overline{\mathrm{H}}, \overline{\mathrm{T}}$ máxima e $\overline{\mathrm{y}}$ máximo com os dois processos fossem concordantes.

Os resultados obtidos em todos os casos estudados permitem concluir que no Processo dos Deslocamentos o número de segmentos suficiente para descrever o comportamento dos cabos submetidos à carregamento uniformemente distribuído em todo o seu vão é igual a metade do número, em metros, do vão destes, como exemplificam os resultados apresentados nas tabelas D.2 e D.3 do Apêndice D.

A comparação dos resultados obtidos com ambos processos comprova que as aproximações feitas no Processo Analítico podem ser realizadas, pois estes são concordantes em todos os casos estudados - como exemplificam os resultados mostrados nas tabelas D.6, D.7 e D.8 onde as variações de $\overline{\mathrm{H}}$, T máxima e ȳ máximo não são superiores a $0,6 \%, 0,7 \%$ e $0,2 \%$, respectivamente.

$\mathrm{Na}$ continuação da divulgação e estudo das coberturas pênseis no Brasil, as formulações e análises apresentadas nesta dissertação podem servir de alicerce para inúmeras pesquisas sobre sistemas estruturais suspensos.

Por serem uma evolução natural deste trabalho, dentre as diversas linhas de pesquisa possíveis, podem-se destacar como bastante interessantes:

- o estudo de até que ponto as expressões do Processo Analítico podem ser simplificadas para tornarem de mais fácil utilização e

- análogo ao apresentado, o estudo pelo Processo Analítico de cabos livremente suspensos submetidos a outros tipos de carregamentos com uma subsequente comparação dos resultados com os obtidos pelo Processo dos Deslocamentos. 


\section{BIBLIOGRAFIA}

ALLESSI,R. et al. (1979). The roof structures of the new sports arena in Atenas. In: WORD CONGRESS ON SHELL AND SPATIAL STRUCTURES, Madrid. Proceedings. p.6.107-6.123

BARBATO, R.L.A. (1975) Contribuição ao estudo das coberturas pênseis em casca aprotendida de revolução. São Carlos, EESC Public., 143p. Tese (Doutorado) - Escola de Engenharia de São Carlos-USP.

BARBATO, R.L.A. (1991) Emprego de cabos livremente suspensos e cabostreliça na construção de coberturas pênseis. São Carlos. Tese (Livre-docência) - Escola de Engenharia de São Carlos-USP.

BARBATO, R.L.A. (1972) Sobre o comportamento estático dos cabos de coberturas pênseis. São Carlos, EESC Public. 89p. Dissertação (Mestrado) Escola de Engenharia de São Carlos-USP.

BERGER, H. \& DE PAOLA, E.M. (1992) Tensile terminal. Civil Engineering, New York, v.62, n.11, p.40-43, Nov.

BUCHHOLDT, H.A. (1985) An introduction to cable roof structures. Cambridge, Cambridge University press, $257 \mathrm{p}$.

DEBS, A.L.H.C. (1983) Contribuição ao estudo das coberturas pênseis com cabos-treliça. São Carlos, EESC Publ. 144p. Dissertação (Mestrado) - Escola de Engenharia de São Carlos-USP. 
HABER, R.B. \& ABEL, J.F. (1982a) Initial equilibrium solution methods for cable reinforced membranes. Part 1 : formulations. Comp. Meths. Appl. Mech. Eng., v.30, p.263-284

HABER, R.B. \& ABEL, J.F. (1982b) Initial equilibrium solution methods for cable reinforced membranes. Part 2 : formulations. Comp. Meths. Appl. Mech. Eng., v.30, p.263-284

IRVINE, H.M. (1981) Cable structures. Cambridge, Ma./London, The MIT Press. 259 p.

KAWAGUCHI, M. (1991) Design problems of long span spatial structures. Engineering Structures, v.13, n.2, p.144-163, Apr.

KRISHNA, P. (1978). Cable-suspended roofs. New York, McGraw-Hill. 314p.

LAN, T.T. (1986) A review of recent developments of spatial structures in China. Bulletin IASS, v.27-2, n.91, p. 51-57, August.

MAJOWIECKI, M. (1985) Tensostrutture: projeto e verifica. Milano, Cisia.

MAJOWIECKI, M. \& OSSOLA, F. (1989) A new stadium for the 1990 world football games. In: 10 YEARS OF PROGRESS IN SHELL AND SPATIAL STRUCTURES, 30th Aniversary of IASS, Madrid. Proceedings.

MAJOWIECKI, M. (1986) Recent developments of design and construction of membrane structures in Italy. In: IASS SYMPOSIUM ON MEMBRANE STRUCTURES AND SPACE FRAMES, Osaka. Proceedings. p.103-110 
OSHIMA, E. (1987) Cálculo de cabo-treliça mediante o emprego de computador. São Carlos. 135p. Dissertação (Mestrado) - Escola de Engenharia de São CarlosUSP.

SALES, J.J. (1988) Projeto e viabilidade econômica de coberturas pênseis com cabos-treliça. São Carlos. 151p. Dissertação (Mestrado) - Escola de Engenharia de São Carlos-USP.

SHEN, S. Z. et al. (1989) Design of two space structures for gymnasiums for Beijing 1990 Asian Games. In: 10 YEARS OF PROGRESS IN SHELL AND SPATIAL STRUCTURES, 30th Anniversary of IASS, Madrid. Proceedings.

SCHLAICH, J. \& BERGERMANN, R. (1984) Recent aplications of light-weigtstructures (cables and metal sheells as tension elements). In: IASS SYMPOSIUM ON SPATIAL ROOF STRUCTURES, Dortmund. Proceedings.

SCHLAICH, J. \& SEIDEL, J. (1985). Die Eislaufhalle in Olympiapark München . Bauingeniur, v.60, p.291-296 


\section{APÊNDICE A}

\section{PROPRIEDADES DOS CABOS DE AÇO NACIONAIS}

\section{A.1 FATOR PARA CÁlCULO DA ÁREA METÁliCA DE UM CABO DE AÇO}

A área metálica de um cabo de aço pode ser calculada, de maneira aproximada, da seguinte forma:

$$
A=f d^{2}
$$

onde: $\mathrm{A}$ = área metálica aproximada; $\mathrm{f}=$ fator de multiplicação que varia em função do tipo de construção do cabo (tabela A.1.1) e d=diâmetro nominal do cabo.

Tabela A.1.1- Valores do fator de multiplicação para o cálculo da área metálica aproximada de cabos de aço.*

\begin{tabular}{|c|l|}
\hline F & Tipo de construção do cabo de aço \\
\hline 0,352 & $\begin{array}{l}8 \times 19 \text { Warrington } \\
8 \times 19 \text { Seale }\end{array}$ \\
\hline 0,380 & $6 \times 7$ \\
\hline 0,395 & $\begin{array}{l}6 \times 9 \text { Warrington } \\
6 \times 19 \text { Seale, } 6 \times 21 \text { failer }\end{array}$ \\
\hline 0,400 & $6 \times 37$ Warrington \\
\hline 0,405 & $\begin{array}{l}6 \times 25 \text { filler, } 6 \times 31 \text { WS, 6x36 WS } \\
6 \times 41 \text { filler, } 6 \times 41 \text { WS }\end{array}$ \\
\hline 0,580 & $1 \times 19$ cordoalha \\
\hline 0,592 & $1 \times 37$ cordoalha \\
\hline 0,596 & $1 \times 7$ cordoalha \\
\hline
\end{tabular}

* 1) Cabos com 6 pernas com AACl adicionar 15\%, à área metálica, com AA adicionar 20\%;

2) Cabos com 8 pernas com AACl adicionar $20 \%$ à área metálica;

3) WS = construção mista Warrington - Seale 


\section{A.2 MÓDULO DE ELASTICIDADE}

Tabela A.2.1- Módulos de elasticidade - cabos com alma de aço.

\begin{tabular}{|c|c|c|}
\hline \multirow{2}{*}{ Classificação } & \multicolumn{2}{|c|}{ Módulo de Elasticidade $\left(\mathrm{Kgf} / \mathrm{mm}^{2}\right)$} \\
\hline & Cabo Normal & Cabo Pré-Estirado \\
\hline $6 \times 7$ & 10500 a 11500 & 13000 \\
\hline $6 \times 19$ & 10000 a 11000 & 12500 \\
\hline $6 \times 37$ & 9500 a 10500 & 12000 \\
\hline 7 fios - cordoalhas & 14500 a 15500 & 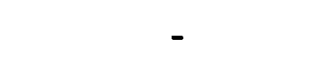 \\
\hline 19 fios - cordoalhas & 13000 a 14000 & 16000 \\
\hline 27 fios - cordoalhas & 12000 a 13000 & 15000 \\
\hline
\end{tabular}




\section{A.3 CARGAS DA RUPTURA E PESO PRÓPRIO DE CABOS DE AÇO}

Tabela A.3- Cabo de aço polido - cargas ruptura e poso próprio - cabos com AACI - classificação 6x19 e 6x37."

\begin{tabular}{|c|c|c|c|c|}
\hline Diâmetro & Nominal & Peso & Carga de & Ruptura $P_{r}$ (tf) \\
\hline (pol) & $(\mathrm{mm})$ & $\mathrm{kgf} / \mathrm{m}$ & $180 \mathrm{a} 200 \mathrm{kgf} / \mathrm{mm}^{2}$ & $200 \mathrm{a} 230 \mathrm{kgf} / \mathrm{mm}^{2}$ \\
\hline $1 / 4$ & 3,20 & 0,171 & 2,66 & 3,06 \\
\hline $5 / 16$ & 7,94 & 0,267 & 4,15 & 4,78 \\
\hline $3 / 8$ & 9,53 & 0,382 & 5,94 & 6,85 \\
\hline $7 / 16$ & 11,11 & 0,528 & 8,06 & 9,25 \\
\hline $1 / 2$ & 12,70 & 0,684 & 10,41 & 12,07 \\
\hline 9/16 & 14,29 & 0,878 & 13,11 & 15,24 \\
\hline $5 / 8$ & 15,28 & 1,071 & 16,23 & 18,69 \\
\hline $3 / 4$ & 19,05 & 1,548 & 23,22 & 26,67 \\
\hline $7 / 8$ & 22,22 & 2,113 & 31,39 & 36,11 \\
\hline 1 & 25,40 & 2,753 & 40,74 & 46,90 \\
\hline $11 / 8$ & 28,58 & 3,482 & 51,28 & 58,97 \\
\hline $11 / 4$ & 31,75 & 4,300 & 62,99 & 72,49 \\
\hline $11 / 3$ & 34,93 & 5,208 & 75,79 & 87,09 \\
\hline $11 / 2$ & 38,10 & 6,190 & 89,76 & 103,42 \\
\hline $15 / 8$ & 41,28 & 7,251 & 104,40 & 119,75 \\
\hline $13 / 4$ & 44,45 & 8,428 & 120,40 & 138,50 \\
\hline $17 / 8$ & 47,63 & 9,653 & 137,60 & 157,85 \\
\hline 2 & 50,80 & 11,005 & 155,87 & 179,63 \\
\hline $21 / 8$ & 53,98 & 12,425 & 174,15 & 200,49 \\
\hline $21 / 4$ & 57,15 & 13,928 & 194,57 & 224,07 \\
\hline $23 / 8$ & 60,515 & 15,515 & 210,00 & 240,00 \\
\hline $21 / 2$ & 63,50 & 17,193 & 235,00 & 265,00 \\
\hline
\end{tabular}

" -Os cabos com diámetro acima de 1 1/2" na faixa de tensões de 200 a $230\left(\mathrm{Kgf} / \mathrm{mm}^{2}\right)$ săo fabricados na classificação $6 \times 37$;

- O diâmetro de $21 / 2$ " é fabricado na classificação $6 \times 37$, nas duas faixas de tensōes. 


\section{APÊNDICE B}

\section{SISTEMA PARA ANÁlISE DE CABOS LIVREMENTE SUSPENSOS COM O EMPREGO DO PROCESSO ANALÍTICO}

\section{B.1 DESCRIÇÃO DO SISTEMA}

\section{B.1.1 INTRODU ÇÃo}

Utilizando o Processo Analítico, preparou-se um sistema de cálculo para microcomputadores em linguagem FORTRAN.

O sistema analisa cabos livremente suspensos submetidos a carregamentos uniformemente distribuídos em todo o vão e a variação de temperatura, desde que as características geométricas iniciais - vão, coordenadas no meio do vão, coordenadas dos apoios e área da seção transversal - características físicas módulo de elasticidade e coeficiente de dilatação térmica - e as ações externas destes cabos sejam previamente especificadas.

\section{B.1.2 PROGRAMA PRINCIPAL}

O programa principal faz a leitura e o relatório dos dados de entrada; executa cálculos preliminares, cálculos para a determinação da configuração inicial de equilíbrio, cálculos para a determinação da configuração final de equilíbrio; e faz relatório dos resultados da análise.

Para isso, emprega as subrotinas LDADOS, CALCH e CALCVU, descritas a seguir. 


\section{B.1.3 SUBROTINA LDADOS}

Esta subrotina lê os nomes dos arquivos de dados através do console do sistema. Estes arquivos são :

- ARQUIVO DE ENTRADA DE DADOS : arquivo old que contém os dados necessários ao cálculo do cabo;

- ARQUIVO DE SAÍDA DE DADOS : arquivo new onde são escritos os dados de leitura e os resultados da análise.

Faz, ainda, a leitura dos outros dados de entrada no arquivo de entrada de dados e gera relatório destes dados no arquivo de saída de dados.

\section{B.1.4 SUBROTINA CALCH (P2)}

Calcula o esforço horizontal no cabo para o carregamento final, P2. Emprega as subrotinas INTEGRA e CALCREC.

\section{B.1.5 SUBROTINA CALCVU ( $\mathrm{X}, \mathrm{VLINHA,V,ULINHA,U)}$}

Calcula os deslocamentos $\mathrm{e}$ as variações dos deslocamentos - V, U,VLINHA, ULINHA - do ponto de abscissa X.

Emprega as subrotinas CALCF e INTEGRA.

\section{B.1.6 SUBROTINA INTEGRA ( a, b, ERRO, N, A2 )}

Efetua integração numérica (Método de Romberg ) no intervalo [ $\mathrm{a}, \mathrm{b}$ ]. O número máximo de divisores é $2^{\mathrm{N}}$; ERRO é a condição de erro para a determinação do número de divisores e A2 é o valor da integral.

Utiliza a subrotina CALCF para o cálculo da função a integrar. 


\section{B.1.7 SUBROTINA CALCF ( $\mathrm{X}, \mathrm{F}$ )}

Calcula o valor da função

$$
f(X)=\frac{\left(1+y^{\prime 2}\right)^{3 / 2}}{E A+H \sqrt{1+y^{/ 2}}}
$$

onde: y' é uma variável auxiliar em função de $X ; F=f(X)$ é o valor da função no ponto $\mathrm{X}$ e, $\mathrm{E}, \mathrm{A}$ e $\mathrm{H}$ são constantes da função.

\section{B.1.8 SUBROTINA CALCREC ( A,B,C,D,N,X )}

Calcula as raizes reais exatas de uma equação cúbica na forma canônica, onde: $\mathrm{A}, \mathrm{B}, \mathrm{C}$ e $\mathrm{D}$ são os coeficientes da equação cúbica; $\mathrm{N}$ é o núnero de raizes reais da equação e, $X$ é o vetor com as raizes reais da equação.

Emprega a subrotina CUR que calcula a raiz cúbica de um número real.

\section{B.1.9 SUBROTINA CUR $(X, Y)$}

Calcula o valor da raiz cúbica, $\mathrm{Y}$, do número real, $\mathrm{X}$.

\section{B.2 DESCRIÇÃO COMPLEMENTAR : O ARQUIVO DE ENTRADA DE DADOS}

O arquivo de entrada de dados deve ser preparado, previamente à execução do sistema, contendo os dados do cabo na forma sequencial.

Apresenta-se, a seguir, a estrutura deste arquivo sequencial : 


\section{NOME\$}

\$1) Número de nós

$\mathbf{N}$

\$ 2 ) Número de carregamento distribuídos NCD

$\$ 3$ ) Coordenadas $x$ e $y$ dos apoios $A$ e $B$

$\mathbf{X A}, \mathbf{Y A}$

$X B, Y B$

\$4) Deslocamentos dos apoios A e B

UA, VA

UB, VB

\$ 5 ) Coordenada inicial y no meio do vão do cabo indeformado

$\mathbf{F}$

\$ 6 ) Área da seção transversal do cabo

A

\$ 7 ) Módulo de elasticidade e coeficiente de dilatação térmica do material do cabo

E, ALFA

\$ 8 ) Carregamento uniformemente distribuído oa longo de todo o vão do cabo

$P(1)$

$P(2)$

P(NCD)

\$ 9 ) Variação de temperatura

DELTAT

O símbolo " \$ " quer dizer " linha para comentário " e as variáveis empregadas estão definidas no item B.3.

A figura B.2.1 auxilia a compreensão do arquivo de entrada de dados.

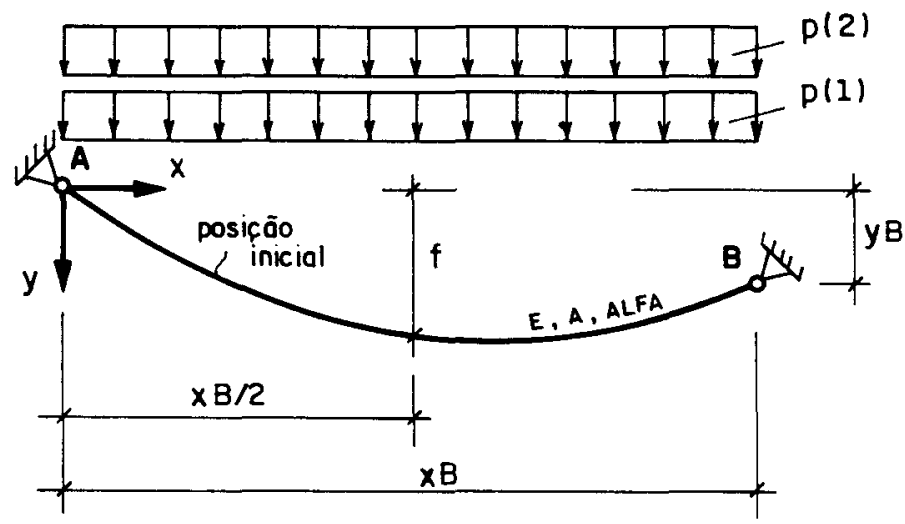

Figura B.2.1 - Ilustração das variáveis do arquivo de entrada de dados. 


\section{B.3 VARIÁ VEIS UTILIZADAS NO SISTEMA}

\section{B.3.1 - PROGRAMA PRINCIPAL}

\section{Variáveis inteiras :}

I,J : contadores

$\mathrm{N} \quad$ : número de pontos nodais a calcular as características do cabo

NCD : número de carregamento

\section{Variáveis reais :}

a : variável auxiliar

ALFA : coeficiente linear de dilatação térmica do material

D : desnível entre os apoios

DELTAT : variação de temperatura

E : módulo de elasticidade do cabo

F : $\quad$ coordenada inicial y no meio do vão (flecha)

H1 : esforço horizontal na seção do cabo, devido carregamento inicial

H2 : esforço horizontal na seção do cabo, devido carregamento final

L : $\quad$ vão do cabo livremente suspenso

$\mathrm{P} \quad$ : vetor com os carregamentos uniformemente distribuídos segundo todo o vão

PH1 : constante do cabo ( relação entre carregamento inicial e H1)

PH2 : constante do cabo ( relação entre carregamento final e H2 )

PP : carregamento final

S : $\quad$ área da seção transversal

$\mathrm{U} \quad$ : $\quad$ vetor com os deslocamentos dos pontos nodais, na direção $\mathrm{x}$

UA,UB : deslocamentos iniciais dos apoios A e B, respectivamente, na direção $\mathrm{x}$

ULINHA : vetor com a variação de $U$ com a variação de $x$

$\mathrm{V} \quad$ : vetor com os deslocamentos dos pontos nodais, na direção y 


$\begin{array}{lll}\text { VA,VB } & : & \begin{array}{l}\text { deslocamentos iniciais dos apoios A e B, respectivamente, na } \\ \text { direção } \mathrm{y}\end{array} \\ \text { VLINHA } & : & \text { vetor com a variação de } \mathrm{V} \text { com a variação de } \mathrm{x} \\ \mathrm{XA}, \mathrm{XB} & : & \text { coordenadas iniciais } \mathrm{x} \text { dos apoios } \mathrm{A} \text { e } \mathrm{B} \text {, respectivamente } \\ \mathrm{XORD} & : & \text { vetor com as coordenadas } \mathrm{x} \text { dos pontos nodais } \\ \text { YA,YB } & : & \text { coordenadas iniciais y dos apoios } \mathrm{A} \text { e } \mathrm{B} \text {, respectivamente } \\ \text { YLINHA } & : & \text { vetor com as tangentes à curva de configuração de equilíbrio, } \\ & & \text { nos pontos nodais } \\ \text { YORD } & : & \text { vetor com as coordenadas y dos pontos nodais } \\ \text { T } & : & \text { vetor com os esforços de tração nodais }\end{array}$

\section{B.3.2 SUBROTINA LDADOS}

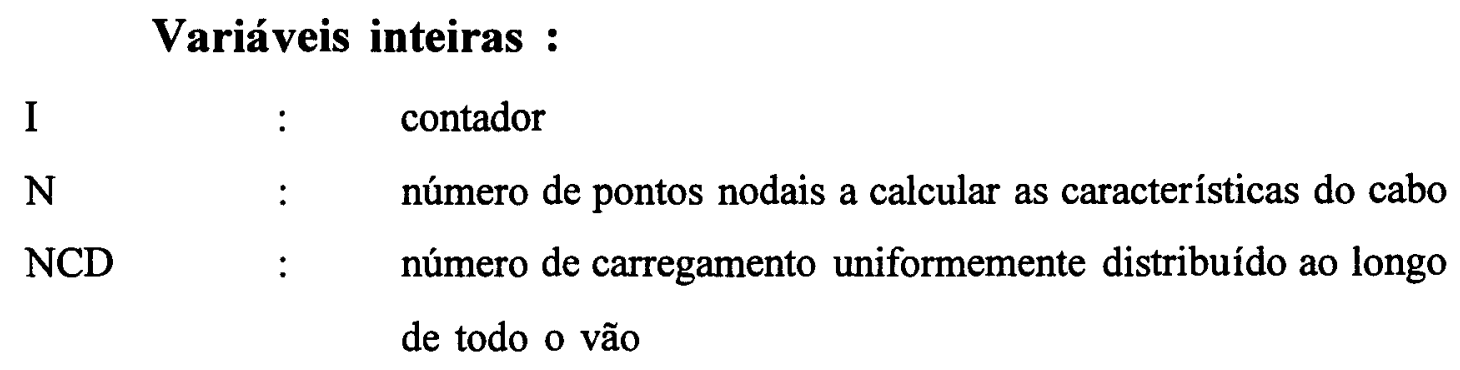

\section{Variáveis reais :}

A : $\quad$ área da seção transversal

ALFA : coeficiente linear de dilatação térmica do material

DELTAT : variação de temperatura

E : módulo de elasticidade do cabo

F : coordenada inicial y no meio do vão (flecha)

P : vetor com os carregamentos uniformemente distribuídos segundo todo o vão

UA,UB : deslocamentos iniciais dos apoios A e B, respectivamente, na direção $\mathrm{x}$

VA,VB : deslocamentos iniciais dos apoios A e B, respectivamente, na direção y

$\mathrm{XA}, \mathrm{XB} \quad$ : coordenadas $\mathrm{x}$ dos apoios $\mathrm{A}$ e $\mathrm{B}$, respectivamente 
YA, YB : coordenadas y dos apoios A e B, respectivamente

\section{Variáveis do tipo caracter :}

AUX : variável auxiliar

NOME1 : $\quad$ arquivo old para leitura de dados

NOME2 : arquivo new para saída de dados

NOME\$ : nome do cabo

\section{B.3.3 SUBROTINA CALCH (P2)}

\section{Variáveis inteiras :}

$\mathrm{N} \quad$ : constante para o cálculo da subrotina INTEGRA

NR : número de raizes reais da equação cúbica para o cálculo de $\mathrm{H} 2$

\section{Variáveis reais :}

a : variável auxiliar

ALFA : coeficiente linear de dilatação térmica

A1,A2 : variáveis auxiliares

AA,BB,CC,DD : $\quad$ coeficientes da equação cúbica para o cálculo de H2

B1,B2 : variáveis auxiliares

$\mathrm{C} 1, \mathrm{C} 2 \quad$ : variáveis auxiliares

D : desnivel entre os apoios

D1,D2,D3 : variáveis auxiliares

DELTAT : variação de temperatura

E : módulo de elasticidade do cabo

E1,F1 : variáveis auxiliares

ERRO : constantes para o cálculo da subrotina INTEGRA

H1 : esforço horizontal na seção do cabo, devido carregamento inicial

H2 : força horizontal na seção do cabo, devido carregamento final 


$\begin{array}{lll}\text { k,w } & : & \text { limites de integração na subrotina INTEGRA } \\ \text { L } & : & \text { vão do cabo livremente suspenso } \\ \text { P2 } & : & \text { carregamento atual } \\ \text { PH1 } & : & \text { constante do cabo (relação entre carregamento inicial e H1) } \\ \text { R } & : & \text { vetor com as raizes reais da equação cúbica para o cálculo de } \\ & & \text { H2 } \\ \text { S } & : & \text { área da seção transversal } \\ \text { UA,UB } & : & \text { deslocamentos iniciais dos apoios A e B, respectivamente, na } \\ & & \text { direção } x \\ \text { VA,VB } & : & \text { deslocamentos iniciais dos apoios A e B, respectivamente, na } \\ & & \text { direção y }\end{array}$

\section{B.3.4 SUBROTINA CALCVU (X,VLINHA,V,ULINHA,U)}

\section{Variáveis inteiras :}

\section{Variáveis reais :}

a : variável auxiliar

ALFA : coeficiente linear de dilatação térmica do material

D : desnível entre os apoios

DELTAT : variação de temperatura

E : módulo de elasticidade do cabo

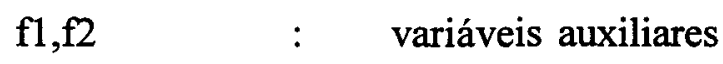

H1 : esforço horizontal na seção do cabo, devido ao carregamento inicial

H2 : esforço horizontal na seção do cabo, devido ao carregamento final

I1,I2,I3,I4,I5,I6 : variáveis auxiliares

L : vão do cabo livremente suspenso

PH1 : constante do cabo ( relação entre carregamento inicial e H1)

PH2 : constante do cabo ( relação entre carregamento final e H2) 
: $\quad$ área da seção transversal

$\mathrm{U}$

UA,UB : deslocamentos iniciais dos apoios A e B, respectivamente, na direção $x$

ULINHA : variação de $\mathrm{U}$ com a variação de $\mathrm{x}$

$\mathrm{V} \quad$ : deslocamento do ponto nodal, na direção y

VA,VB : deslocamentos conhecidos dos apoios A e B, respectivamente, na direção y

VLINHA : variação de $V$ com a variação de $x$

$\mathrm{X} \quad$ : coordenada $\mathrm{x}$ do ponto a calcular ULINHA,U,VLINHA, $\mathrm{V}$

YLINHA : tangente à curva de configuração de equilíbrio no ponto a calcular ULINHA,U,VLINHA,V

w,ERRO : constantes para o cálculo da subrotina INTEGRA

\section{B.3.5 SUBROTINA INTEGRA (a,b,ERRO,N,A2)}

\section{Variáveis inteiras :}

I,J : contadores

$\mathrm{N} \quad: \quad 2^{\mathrm{N}}$ é o número máximo de divisores

LOOP : número atual de divisores

\section{Variáveis reais :}

a,b : intervalo de integração

A1 : valor aproximado da área com o número de divisores iqual a $2^{(\mathrm{N}-1)}$

A2 : valor aproximado da área com o número de divisores iqual a $2^{\mathrm{N}}$

ERRO : condição de erro para a determinação do número de divisores

$$
\text { ERRO }>\mid \mathrm{A} 2 \text { - A1 } \mid
$$

: valor da função no ponto inicial de um intervalo

F2 : valor da função no ponto final de um intervalo

$\mathrm{X} \quad$ : $\quad$ intervalo entre divisores 
XORD : coordenada $\mathrm{x}$ de um ponto a calcular a função

\section{B.3.6 SUBROTINA CALCF $(\mathbf{X , F})$}

Variáveis reais :

E,S,L,D,H1,PH1 : constantes da função a calcular

$\mathrm{F} \quad$ : valor da função no ponto $\mathrm{X}$

$\mathrm{X} \quad$ : valor da variável da função

YLINHA : variável auxiliar

\section{B.3.7 SUBROTINA CALCREC (A,B,C,D,N,X)}

\section{Variáveis inteiras :}

$\mathrm{N} \quad$ : número de raizes reais da equação cúbica

\section{Variáveis reais :}

A,B,C,D : coeficientes da equação cúbica a calcular, na forma canônica

$$
\left(\mathrm{AX}^{3}+\mathrm{BX} \mathrm{X}^{2}+\mathrm{CX}+\mathrm{D}=0\right)
$$

$\mathrm{X} \quad$ : vetor com as raizes reais da equação cúbica a calcular

$\mathrm{P}, \mathrm{Q} \quad$ : coeficiente da equação auxiliar $\quad\left(\mathrm{Y}^{3}+3 \mathrm{PY}+2 \mathrm{Q}=0\right)$

TETA,U,V : variáveis auxiliares

ALFA,R,DD : variáveis auxiliares

\section{B.3.7 - SUBROTINA CUR(X,Y)}

\section{Variáveis reais :}
AUX
: váriável auxiliar
$\mathrm{X}$
: número a calcular a raiz cúbica
$\mathrm{Y}$
: valor calculado da raiz cúbica de $\mathrm{X}$ 


\section{B.4 PROGRAMA FONTE}

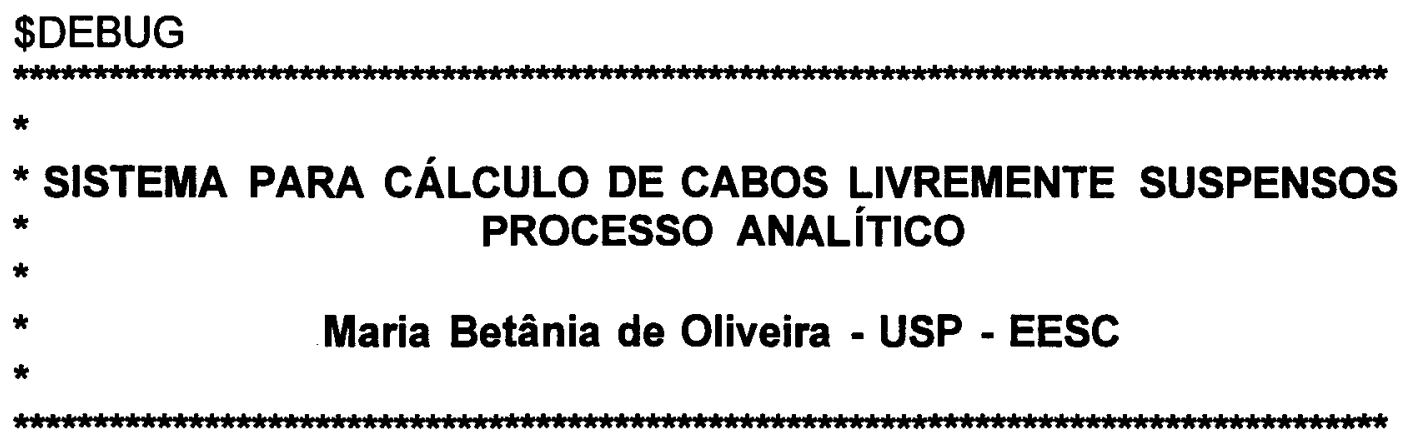

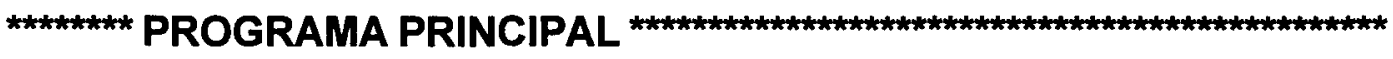

* a) Declaração das variáveis

INTEGER I,J,N,NCD

DOUBLE PRECISION a,ALFA,D,DELTAT,E,F,H1,H2,L,P(10),PH1, ${ }^{*} P H 2, P P, S, U(100), U A, U B, U L I N H A(100), V(100), V A, V B, V L I N H A(100)$, ${ }^{*} X A, X B, X O R D(100), Y A, Y B, Y L I N H A(100), Y O R D(100), T(100)$

* b) Definição de área de memória

COMMON/BLOC1/S,E

COMMON/BLOC2/L,D,VA,VB,UA,UB

COMMON/BLOC3/ALFA,DELTAT

COMMON/BLOC4/H1,H2,PH1,PH2

COMMON/BLOC5/XA, YA,XB, YB,F

COMMON/BLOC6/N,NCD

COMMON/BLOC7/P

* C) Leitura e impressão dos dados de entrada

CALL LDADOS

* d) Cálculos preliminares

$L=X B-X A$

$D=Y B-Y A$

* e) Cálculo da posição inicial de equilíbrio, $p=0$ e $\mathrm{H=0}$

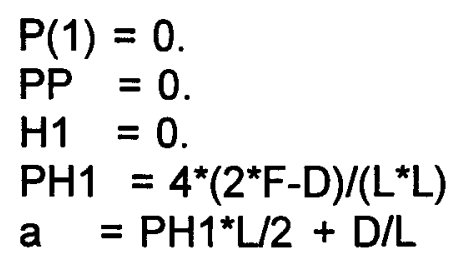


WRITE $(3,10)$ PP,H1,PH1

DO $1000, I=1, N$ IF (I.EQ.1)

THEN

$$
\begin{aligned}
& \mathrm{XORD}(\mathrm{I})=0 . \\
& \text { ELSE } \\
& \mathrm{XORD}(\mathrm{I})=\mathrm{XORD}(\mathrm{I}-1)+\mathrm{L}(\mathrm{N}-1) \\
& \text { ENDIF } \\
& \text { YLINHA }(I)=-P H 1^{*} X O R D(I)+a \\
& \text { YORD }(I)=-P H 1^{*}\left(X O R D(I)^{\star \star} 2\right) / 2+a^{*} X O R D(I) \\
& \text { WRITE }(3,15) \text { I,XORD(I),YORD(I),YLINHA(I) }
\end{aligned}
$$

1000 CONTINUE

10 FORMAT $(I, 6 X, ' 6)$ POSICAO INICIAL DE EQUILIBRIO ',I,6X,' p:' ",F10.2,3X,' H : ',F15.5,3X,' p/H :',F10.6,II,7X,' No ',17X,' X',17X, *' $\left.Y^{\prime}, 16 X, Y^{\prime} Y^{\prime}, '\right)$

15 FORMAT ( $6 \mathrm{X}, 13,3 \mathrm{X}, \mathrm{F} 15.8,3 \mathrm{X}, \mathrm{F} 15.8,3 \mathrm{X}, \mathrm{F} 15.8)$

f)Cálculo da posição atual de equilíbrio

WRITE $(3,25)$

DO $1010, \mathrm{~J}=2, \mathrm{NCD}+1$

$P P=P P+P(J)$

write $\left({ }^{*},{ }^{*}\right)$ PP

read $\left({ }^{*}, *\right)$

f.1) Cálculo de H2

CALL CALCH $(P(J))$

f.2) Cálculo de V,VLINHA,U,ULINHA

$\mathrm{PH} 2=\mathrm{P}(\mathrm{J}) / \mathrm{H} 2$

DO $1020, I=1, N$

CALLCALCVU (XORD(I),VLINHA(I),V(I),ULINHA(I),U(I)) CONTINUE

f.3) Cálculo de $Y, X$, YLINHA e $T$ finais

$\mathrm{PH} 1=\mathrm{PP} / \mathrm{H} 2$

$a=P H 1 * L / 2+D / L$

WRITE $(3,30)(\mathrm{J}-1), \mathrm{P}(\mathrm{J}), \mathrm{H} 2, \mathrm{PH} 2$

DO $1030, I=1, N$

$\operatorname{XORD}(I)=X O R D(I)+U(I)$

$\mathrm{YORD}(\mathrm{I})=\mathrm{YORD}(\mathrm{I})+\mathrm{V}(\mathrm{I})$

$\mathrm{T}(\mathrm{I})=\mathrm{H} 2^{*} \mathrm{DSQRT}(1+((\mathrm{YLINHA}(\mathrm{I})+\mathrm{VLINHA}(\mathrm{I})) /$

$\left.(1+U L I N H A(I)))^{\star \star} 2\right)$

$\mathrm{YLINHA}(\mathrm{I})=-\mathrm{PH} 1{ }^{*} \mathrm{XORD}(\mathrm{I})+\mathrm{a}$ 


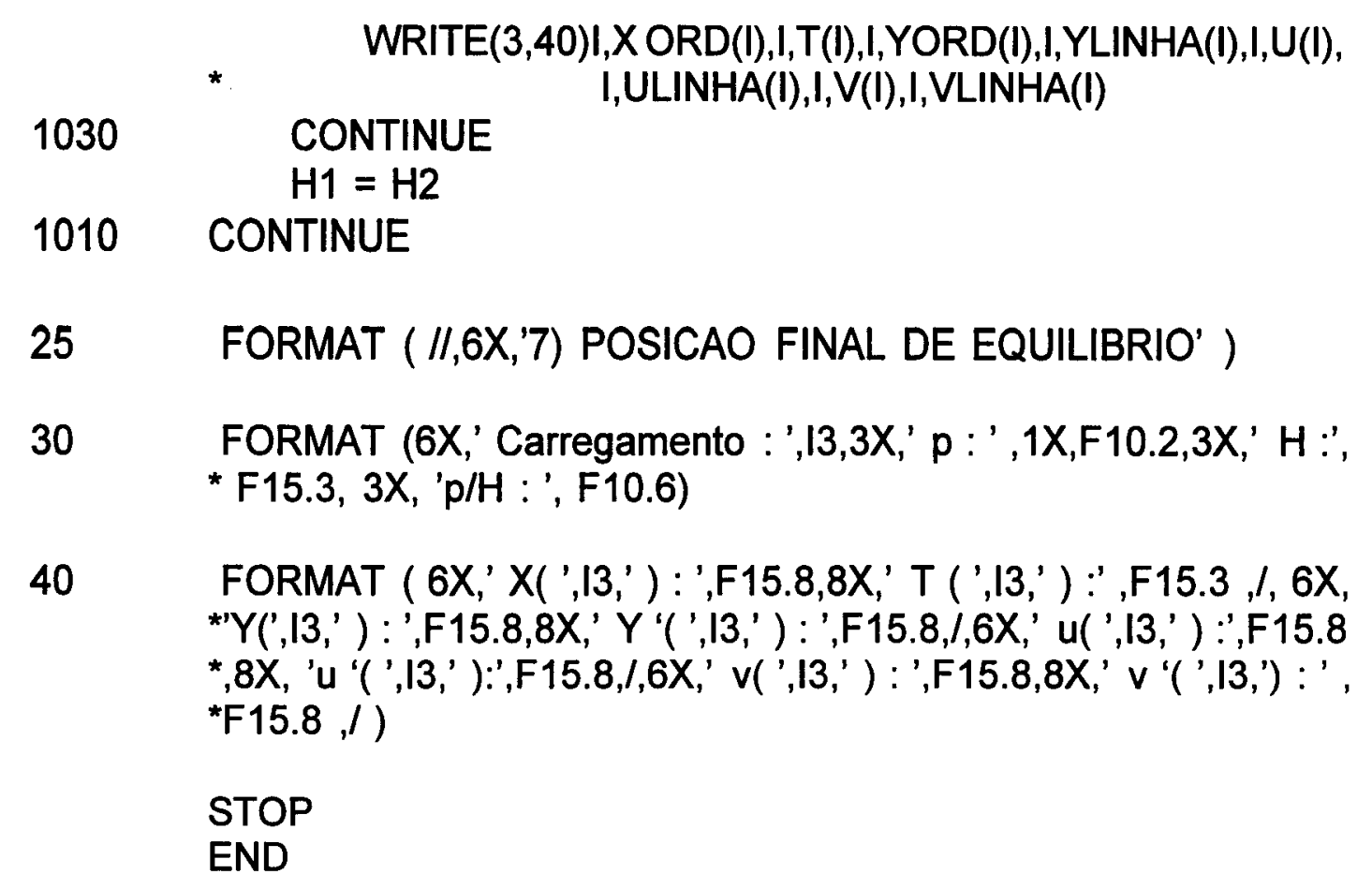

SUBROTINA LDADOS

SUBROUTINE LDADOS

a) Declaração das variáveis

INTEGER I,N,NCD

DOUBLE PRECISION A,ALFA ,D,DELTAT ,E,F,L,P(10),UA ,UB, * VA,VB, XA,XB,YA,YB

CHARACTER $* 7$ NOME1,NOME2

CHARACTER*80 AUX,NOME\$

* b) Definição de área de memória

COMMON/BLOC1/A,E

COMMON/BLOC2/L,D,VA,VB,UA,UB

COMMON/BLOC3/ALFA,DELTAT

COMMON/BLOC5/XA,YA,XB,YB,F

COMMON/BLOC6/N,NCD

COMMON/BLOC7/P

* c) Leitura dos dados

WRITE $(*, 1)$

WRITE $(*, 2)$

WRITE $(*, 5)$

READ $\left({ }^{*}, 6\right)$ NOME1

WRITE $\left({ }^{*}, 8\right)$

READ $(*, 6)$ NOME2 


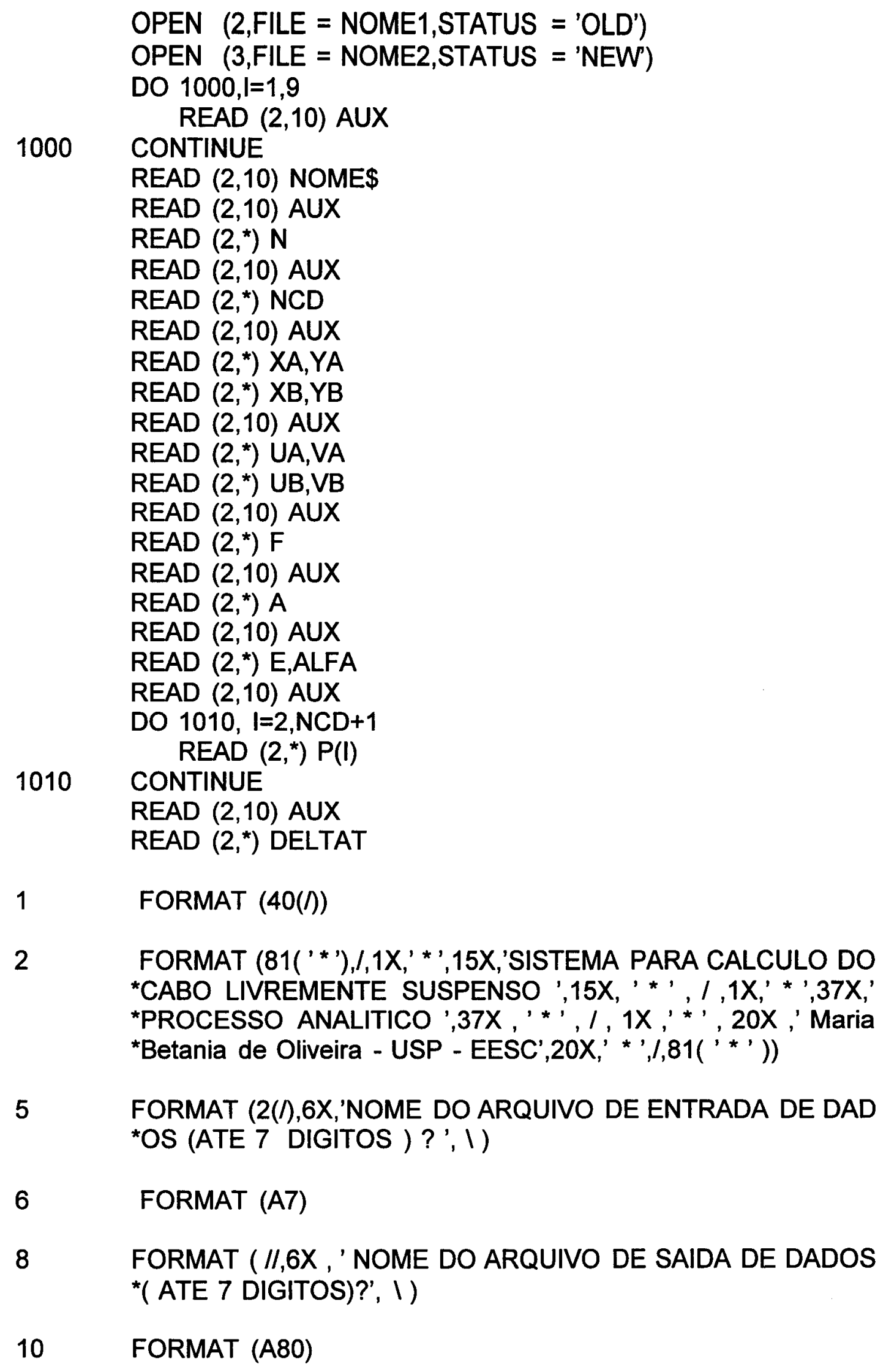

5 FORMAT (2(l),6X,'NOME DO ARQUIVO DE ENTRADA DE DAD ${ }^{*}$ OS (ATE 7 DIGITOS ) ?', I)

$6 \quad$ FORMAT (A7)

8 FORMAT ( $/, 6 \mathrm{X}$, , NOME DO ARQUIVO DE SAIDA DE DADOS *( ATE 7 DIGITOS)?', I) 
d) Emissão do relatório dos dados de entrada

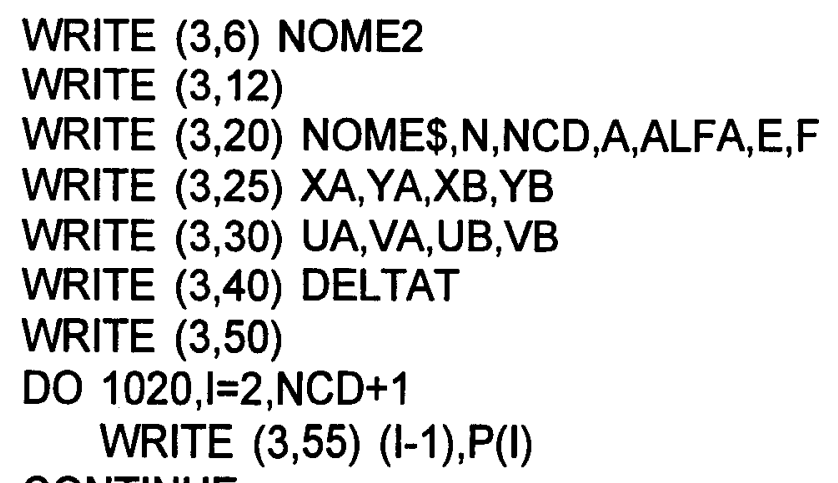

12 FORMAT (80(' * '),l,' * ',78X,' *',l,' * ',15X,'SISTEMA PARA CAL *CULO DE CABOS LIVREMENTE SUSPENSOS ',15 $X,{ }^{\prime}$ *',, *, * ',78X,' * ',l,' * ',37X,' PROCESSO ANALITICO ', 37X,' * ', , I

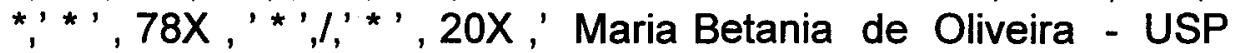

FORMAT ( /l,6X,' NOME DA ESTRUTURA : ', A80,/l,6X,' 1) "DADOS GERAIS DA ESTRUTURA ',I,6X,' Numero de pontos *nodais ',10X, ' : ',13X,13, , 6X, 'Numero de carregamento ', 11X,' *: ',14X, $12, \quad / \quad 6 X, '$ Area da secao transversal',8X,': *',F15.10,I,6X,'Coeficiente de dilatacao termica : ',F15.12 ,I, 6X, *'Modulo de elasticidade',11X,': ',F15.2,I,6X,'Flecha inicial do cabo *', 11X,' : ', F15.3)

25 FORMAT $(/ I, 6 \mathrm{X}, 2)$ COORDENADAS DOS APOIOS ', I, 19X ' $X A$ *', 16X,' YA ',16X,'XB ',16X,' YB ',/,6X,F15.8,3X, F15.8, 3X, F15.8 $\left.{ }^{*}, 3 \mathrm{X}, \mathrm{F} 15.8\right)$

FORMAT( $/ /, 6 X, ' 3)$ DESLOCAMENTOS CONHECIDOS ', I,19X "', uA ',16X,' vA ',16X,' uB',16X,' vB ', I,6X, F15.8, 3X,F15.8 ,3X, ${ }^{*} \mathrm{~F} 15.8,3 \mathrm{X}, \mathrm{F} 15.8$ )

40 FORMAT $(I /, 6 \mathrm{X}$, '4) VARIACAO DE TEMPERATURA', I,6X,'DeltaT * :',F6.3)

FORMAT $(/ /, 6 X, ' 5)$ CARREGAMENTO DISTRIBUIDO SEGUNDO * O VAO')

FORMAT $\left(6 X,{ }^{\prime} p\left({ }^{\prime}, 12,{ }^{\prime}\right)\right.$ : ',F10.2)

RETURN END 


\section{SUBROUTINE CALCH (P2)}

a) Declaração das variáveis

INTEGER N,NR

DOUBLE PRECISIONa,ALFA,A1,A2,AA,BB,CC,DD,B1,B2,C1,C2

* ,D,D1,D2,D3,DELTAT,E,E1,ERRO,F1,H1,H2,k,w,L,P2,PH1,PH2,

* $R(3), S, U A, U B, V A, V B$

b) Definição de área de memória

COMMON/BLOC1/S,E

COMMON/BLOC2/L,D,VA,VB,UA,UB

COMMON/BLOC3/ALFA,DELTAT

COMMON/BLOC4/H1,H2,PH1,PH2

c) Cálculo de H2

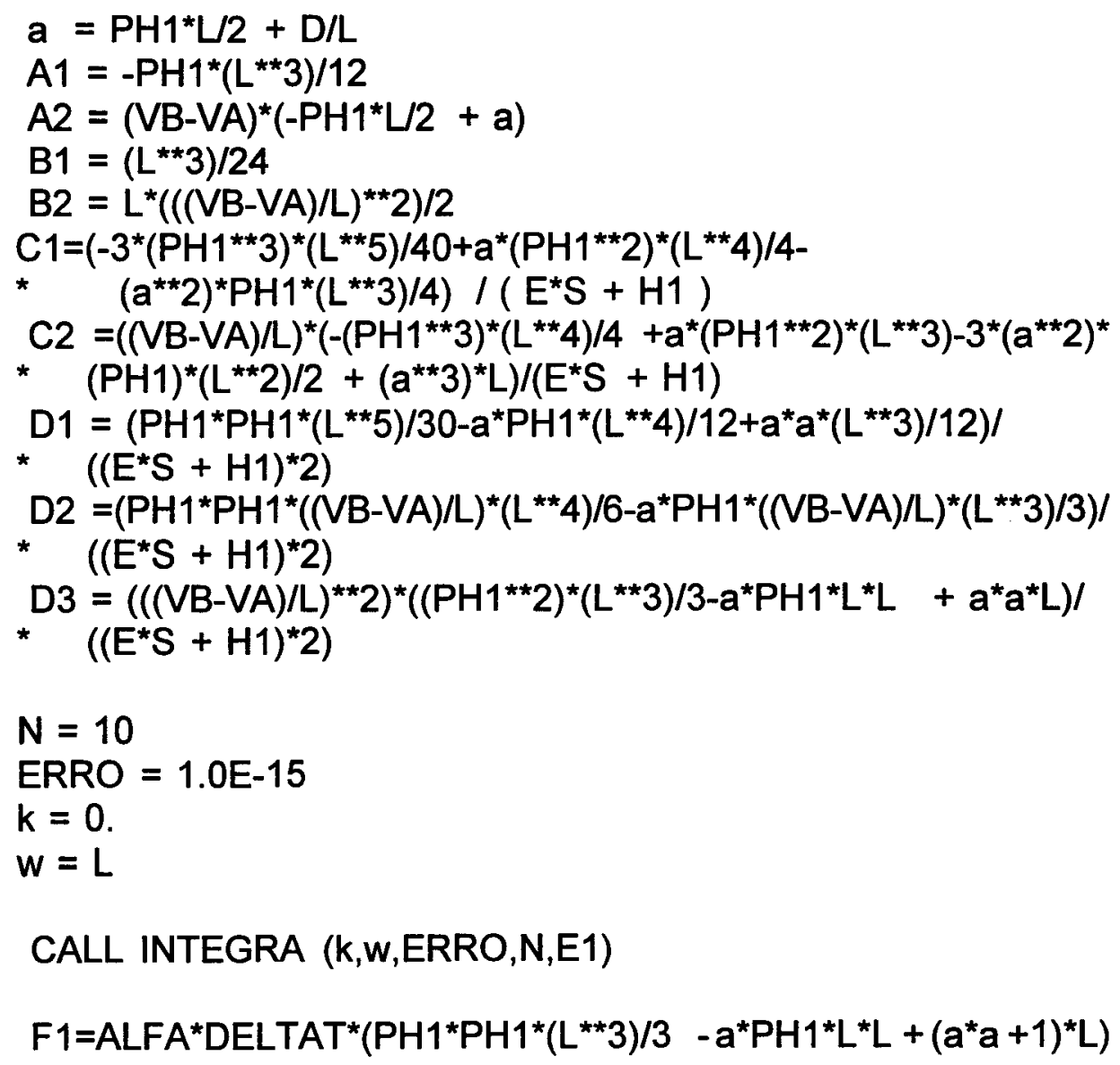

CALL INTEGRA ( $k, w, E R R O, N, E 1)$

$F 1=A L F A^{*} D E L T A T^{*}\left(P H 1^{*} P H 1^{*}\left(L^{* *} 3\right) / 3-a^{*} P H 1^{*} L^{*} L+\left(a^{*} a+1\right)^{*} L\right)$ 


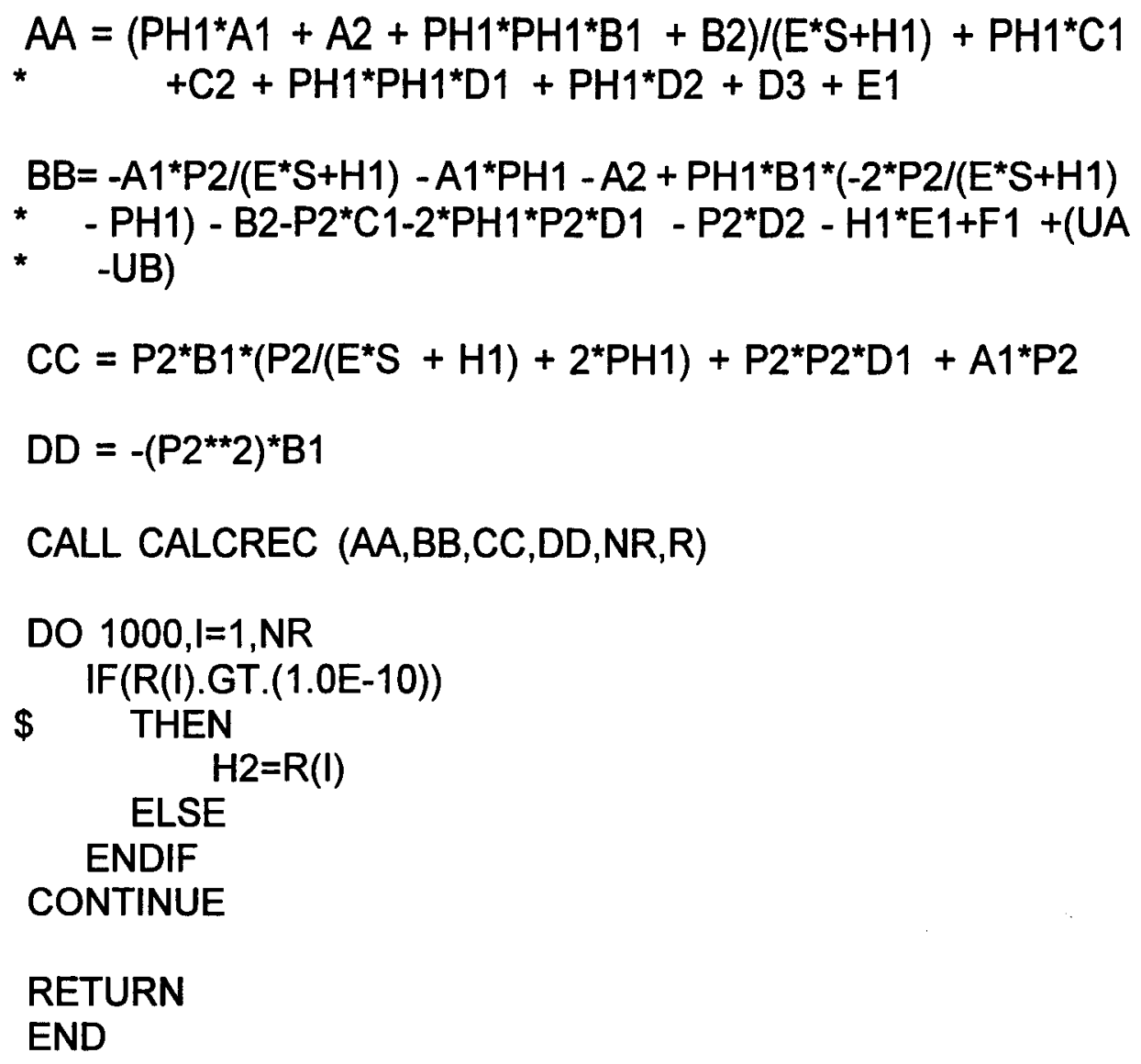

SUBROTINA CALCVU

SUBROUTINE CALCVU (X,VLINHA,V,ULINHA,U)

* a) Declaração das variáveis

INTEGER N

DOUBLE PRECISIONa,ALFA,D,DELTAT,E,ERRO, f1, $\mathrm{f2,H1,H2,I1}$ ${ }^{*}, 12,13,14,15,16, \mathrm{~L}, \mathrm{PH} 1, \mathrm{PH} 2, \mathrm{~S}, \mathrm{U}, \mathrm{UA}, \mathrm{UB}, \mathrm{ULINHA}, \mathrm{V}, \mathrm{VA}, \mathrm{VB}, \mathrm{VLINHA}$, *X, YLINHA,W

* b) Definição de área de memória

COMMON/BLOC1/S,E

COMMON/BLOC2/L,D,VA,VB,UA,UB

COMMON/BLOC3/ALFA,DELTAT

COMMON/BLOC4/H1,H2,PH1,PH2

c) Cálculo de VLINHA e V

VLINHA $=(\mathrm{PH} 1-\mathrm{PH} 2)^{*} \mathrm{X}+(\mathrm{VB}-\mathrm{VA}) / \mathrm{L}-\mathrm{L}^{*}(\mathrm{PH} 1-\mathrm{PH} 2) / 2$ 
$V=(\mathrm{PH} 1-\mathrm{PH} 2)^{*}\left(\mathrm{X}^{* \star} 2\right) / 2+\left((\mathrm{VB}-\mathrm{VA}) / \mathrm{L}-\mathrm{L}^{*}(\mathrm{PH} 1-\mathrm{PH} 2) / 2\right)^{\star} \mathrm{X}+\mathrm{VA}$

d) Cálculo de ULINHA e U

d.1) Cálculo de ULINHA

CALL CALCF $(X, f 1)$

$\mathrm{a}=\mathrm{PH} 1 * \mathrm{~L} / 2+\mathrm{D} / \mathrm{L}$

YLINHA $=-P H 1 * X+a$

f2 = ALFA*DELTAT* $\left(1+Y \operatorname{LINHA}^{* *} 2\right)$

ULINHA $=\left(H 2 /\left(E^{*} S+H 1\right)-1\right)^{*}\left(Y L I N H A * V L I N H A+\left(V L I N H A A^{* \star} 2\right) / 2\right)$

${ }^{*}+\mathrm{H}^{*}\left(\mathrm{VLINHA} \mathrm{A}^{*} \mathrm{YLINHA} \mathrm{A}^{* *} 3+\left(\left(\mathrm{YLINH}{ }^{* *} 2\right)^{*} \mathrm{VLINHA}{ }^{* *} 2\right) / 2\right) /\left(\mathrm{E}^{*} \mathrm{~S}+\right.$

$\left.{ }^{*} H 1\right)+(H 2-H 1)^{*} f 1+f 2$

d.2) Cálculo de U

$11=-\mathrm{PH} 1^{*}(\mathrm{PH} 1-\mathrm{PH} 2)^{*}\left(\mathrm{X}^{\star *} 3\right) / 3+\left(-\mathrm{PH} 1^{*}\left((\mathrm{VB}-\mathrm{VA}) / \mathrm{L}-\mathrm{L}^{*}(\mathrm{PH} 1-\mathrm{PH} 2) / 2\right)\right.$

$\left.{ }^{*}+a^{*}(P H 1-P H 2)\right)^{\star} X^{\star} X / 2+a^{*}\left((V B-V A) / L-L^{*}(P H 1-P H 2) / 2\right)^{\star} X$

$12=\left((\mathrm{PH} 1-\mathrm{PH} 2)^{* *} 2\right)^{*}\left(\mathrm{X}^{* *} 3\right) / 3+(\mathrm{PH} 1-\mathrm{PH} 2) *((\mathrm{VB}-\mathrm{VA}) / \mathrm{L}-$

* $\left.\left.\quad L^{*}(\mathrm{PH} 1-\mathrm{PH} 2) / 2\right)^{*} \mathrm{X}^{\star} \mathrm{X}+\left((\mathrm{VB}-\mathrm{VA}) / \mathrm{L}-\mathrm{L}^{*}(\mathrm{PH} 1-\mathrm{PH} 2) / 2\right)^{\star *} 2\right)^{\star} \mathrm{X}$

$13=(\mathrm{PH} 1-\mathrm{PH} 2)^{*}\left(-\left(\mathrm{PH} 1^{* *} 3\right)^{*}\left(\mathrm{X}^{* *} 5\right) / 5+3^{*} \mathrm{a}^{*}\left(\mathrm{PH} 1^{* *} 2\right)^{*}\left(\mathrm{X}^{* *} 4\right) / 4-\right.$

* $\left.\left(\mathrm{a}^{* *} 2\right)^{*} \mathrm{PH} 1^{*}\left(\mathrm{X}^{\star \star} 3\right)+\left(\mathrm{a}^{* *} 3\right)^{*}\left(\mathrm{X}^{* \star} 2\right) / 2\right)+\left((\mathrm{VB}-\mathrm{VA}) / \mathrm{L}-\mathrm{L}^{*}(\mathrm{PH} 1-\right.$

* $\mathrm{PH} 2) / 2)^{\star}\left(-\left(\mathrm{PH} 1^{\star *} 3\right)^{\star}\left(\mathrm{X}^{\star *} 4\right) / 4+\mathrm{a}^{*}\left(\mathrm{PH} 1^{\star \star} 2\right)^{\star} \mathrm{X}^{\star \star} 3-3^{*}\left(\mathrm{a}^{\star \star} 2\right)^{*}\right.$

* $\left.\mathrm{PH} 1^{\star}\left(\mathrm{X}^{\star \star} 2\right) / 2+\left(\mathrm{a}^{\star \star} 3\right)^{\star} \mathrm{X}\right)$

14=PH1 ${ }^{*} \mathrm{PH} 1^{*}\left((\mathrm{PH} 1-\mathrm{PH} 2)^{\star \star} 2\right)^{\star}\left(\mathrm{X}^{\star *} 5\right) / 5+\mathrm{PH} 1^{*} \mathrm{PH} 1^{*} \quad(\mathrm{PH} 1-\mathrm{PH} 2)^{*}$

* $\quad\left((\mathrm{VB}-\mathrm{VA}) / \mathrm{L}-\mathrm{L}^{*}(\mathrm{PH} 1-\mathrm{PH} 2) / 2\right)^{*}\left(\mathrm{X}^{\star *} 4\right) / 2+\left(\mathrm{PH} 1^{* *} 2\right)^{\star}((\mathrm{VB}-\mathrm{VA}) / \mathrm{L}-$

* $\left.\left.\quad L^{*}(\mathrm{PH} 1-\mathrm{PH} 2) / 2\right)^{\star \star} 2\right)^{\star}\left(\mathrm{X}^{\star \star} 3\right) / 3-\mathrm{a}^{*} \mathrm{PH} 1^{*}\left((\mathrm{PH} 1-\mathrm{PH} 2)^{\star \star} 2\right)^{\star}\left(\mathrm{X}^{\star \star} 4\right) / 2$

* $\left.\quad-4^{*} \mathrm{a}^{*} \mathrm{PH} 1^{*}(\mathrm{PH} 1-\mathrm{PH} 2)^{*}(\mathrm{VB}-\mathrm{VA}) / \mathrm{L}-\mathrm{L}^{*}(\mathrm{PH} 1-\mathrm{PH} 2) / 2\right)^{*}\left(\mathrm{X}^{* *} 3\right) / 3$

* $\quad-a^{*} P H 1^{*}\left(\left(\text { VB-VA)/L-L*(PH1-PH2)/2) }{ }^{\star \star} 2\right)^{*}\left(X^{\star \star} 2\right)+\left(a^{\star *} 2\right)\right.$

* $\quad{ }^{*}\left((\mathrm{PH} 1-\mathrm{PH} 2)^{\star \star} 2\right)^{*}\left(\mathrm{X}^{\star \star} 3\right) / 3+\left(\mathrm{a}^{\star \star} 2\right)^{\star}(\mathrm{PH} 1-\mathrm{PH} 2)^{\star}(\mathrm{VB}-\mathrm{VA}) / \mathrm{L} \quad-$

* $\left.\left.\quad L^{*}(\mathrm{PH} 1-\mathrm{PH} 2) / 2\right)^{\star} X^{\star} X+a^{*} a^{*}\left((\mathrm{VB}-\mathrm{VA}) / \mathrm{L}-\mathrm{L}^{*}(\mathrm{PH} 1-\mathrm{PH} 2) / 2\right)^{\star \star} 2\right)^{\star} X$

$N=10$

$\mathrm{ERRO}=1.0 \mathrm{E}-15$

$w=0$.

CALL INTEGRA (w,X,ERRO,N,I5)

I6 $=$ ALFA $^{*} \operatorname{DELTAT}^{\star}\left(\mathrm{PH}^{*}{ }^{*} \mathrm{PH} 1^{*}\left(\mathrm{X}^{\star *} 3\right) / 3-\mathrm{a}^{*} \mathrm{PH} 1^{*} \mathrm{X}^{*} \mathrm{X}+\left(\mathrm{a}^{*} \mathrm{a}+1\right)^{\star} \mathrm{X}\right)$

$\mathrm{U}=\left(\mathrm{H} 2 /\left(\mathrm{E}^{*} \mathrm{~S}+\mathrm{H} 1\right)-1\right)^{\star} \mathrm{I} 1+\left(\mathrm{H} 2 /\left(\mathrm{E}^{*} \mathrm{~S}+\mathrm{H} 1\right)-1\right)^{\star} \mathrm{I} / 2 \mathrm{2}+\mathrm{H} 2^{\star} \mid 3 /\left(\mathrm{E}^{*} \mathrm{~S}+\mathrm{H} 1\right)$

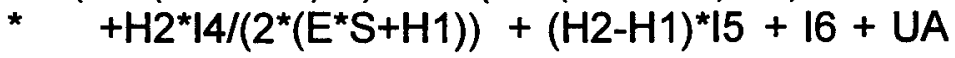

RETURN

END 
SUBROUTINE INTEGRA (a,b,ERRO,N,A2)

*

a) Declaração das variáveis

INTEGER I,J,LOOP,N

DOUBLE PRECISION a,A1,A2,b,ERRO,F1,F2,X,XORD

* b) Cálculo da integração

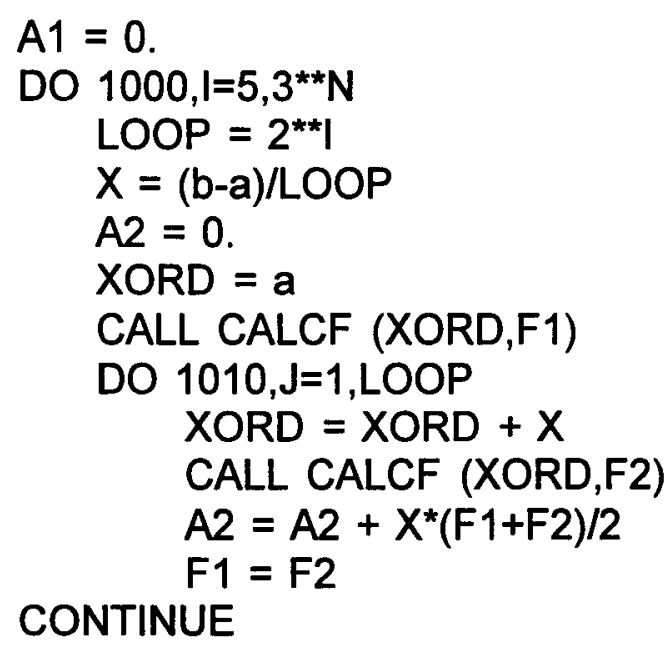

1010 CONTINUE

IF (DABS(A2-A1).LE.ERRO)

$\$$ THEN

write $(*, *) A 2$

RETURN

ELSE

$A 1=A 2$

ENDIF

1000 CONTINUE

WRITE $(*, 10)$

$\operatorname{READ}\left({ }^{*}, *\right)$

10

FORMAT('INTEGRA -Condicao nao satisfeita - pressione ENTER *')

RETURN

END 


\section{SUBROUTINE CALCF $(X, F)$}

a) Declaração das variáveis

DOUBLE PRECISION E,S,L,D,VA,VB,UA,UB,H1,H2,PH1,PH2,X, ${ }^{\star} \mathrm{F}, \mathrm{YLINHA}$

*

b) Definição de área de memória

COMMON/BLOC1/S,E COMMON/BLOC2/L,D,VA,VB,UA,UB COMMON/BLOC4/H1,H2,PH1,PH2

c) Cálculo da função

YLINHA $=-\mathrm{PH}^{*} \mathrm{X}+\mathrm{PH} 1^{*} \mathrm{~L} / 2+\mathrm{D} / \mathrm{L}$

$F=\left(D S Q R T\left(\left(1+Y L_{I N H A}{ }^{\star \star} 2\right)^{\star *} 3\right)\right) /\left(E^{\star} S+H 1^{*}\left(D S Q R T\left(1+Y L I N H A^{\star *}\right.\right.\right.$ *2)))

\section{RETURN}

END

SUBROTINA CALCREC

SUBROUTINE CALCREC (A,B,C,D,N,X)

* a) Declaração das variáveis

INTEGER N

DOUBLE PRECISION A,B,C,D,X(3),DD,R,P,Q,U,V,ALFA,TETA ${ }^{*}, \mathrm{AUX}$

* b) Cálculo das raizes reais

IF (A.EQ.0.)

$\$$ THEN

WRITE $(*, 10)$

ENDIF

10 FORMAT (//,6X,'CALCREC - A equação não é cúbica' )

$$
\begin{aligned}
& P=C /\left(3^{\star} A\right)-\left(B^{\star \star} 2\right) /\left(9^{*} A^{\star \star} 2\right) \\
& Q=\left(B^{\star \star} 3\right) /\left(27^{\star} A^{\star \star} 3\right)-B^{\star} C /\left(6^{\star} A^{\star *} 2\right)+D /\left(2^{\star} A\right)
\end{aligned}
$$




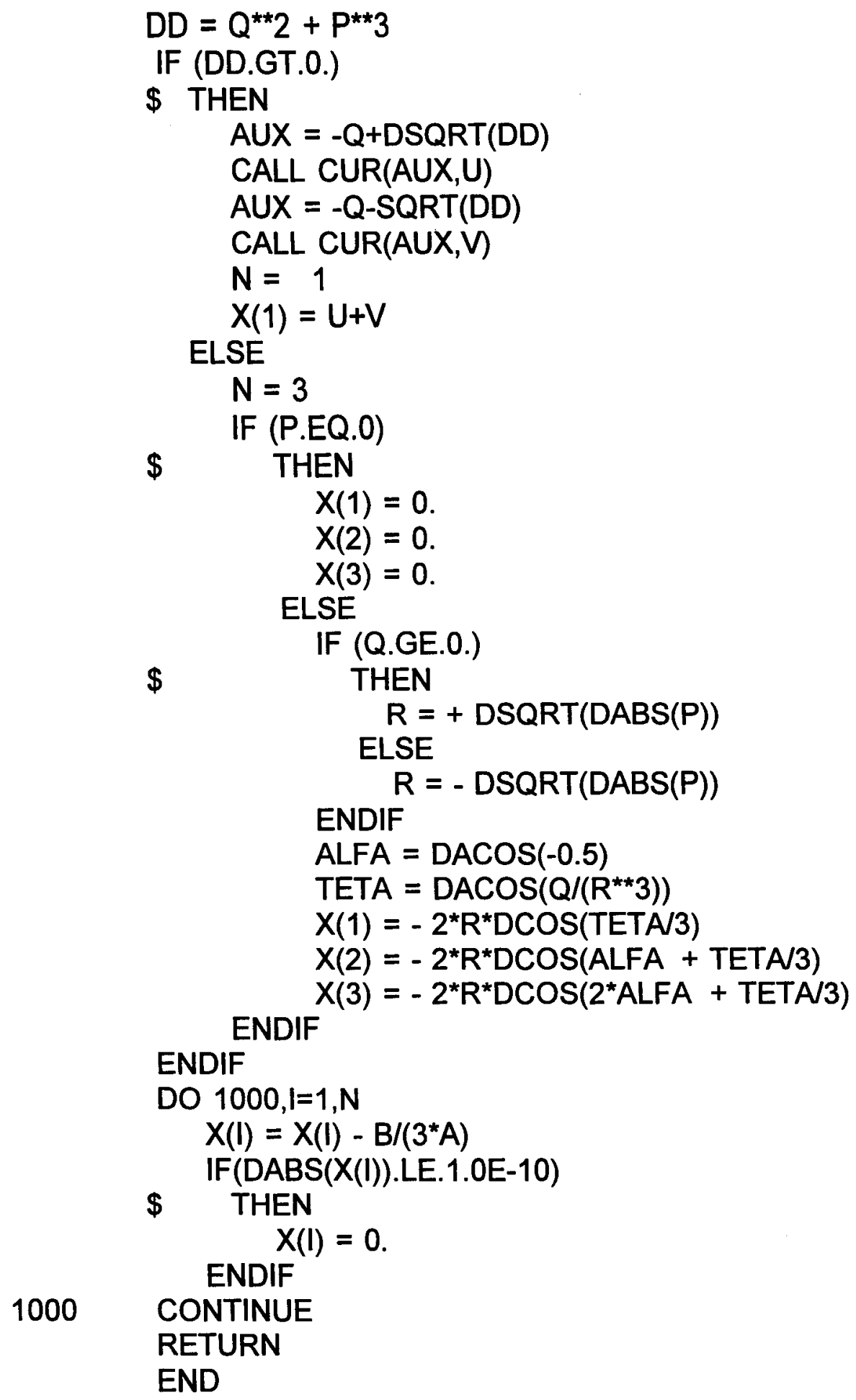

$\$$$$
R=+D S Q R T(D A B S(P))
$$

ENDIF

DO $1000, l=1, N$

$$
X(I)=X(I)-B /\left(3^{*} A\right)
$$

IF(DABS(X(I)).LE.1.0E-10)

$\$$ THEN

$$
X(I)=0 .
$$

1000 CONTINUE

RETURN

END 


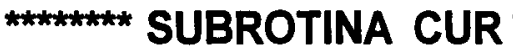

SUBROUTINE CUR(X,Y)

* a) Declaração de variáveis

DOUBLE PRECISION AUX,X,Y

b) Cálculo da raiz cúbica

IF (X.EQ.O.)

$\$$ THEN

$Y=0$.

ELSE

$A U X=D L O G(D A B S(X))$

$\mathrm{Y}=\mathrm{X}^{\star}(\mathrm{DEXP}(\mathrm{AUX} / 3)) / \mathrm{DABS}(\mathrm{X})$

ENDIF

RETURN

END 


\section{APÊNDICE C}

\section{SISTEMA PARA ANÁLISE DE CABOS LIVREMENTE SUSPENSOS COM O EMPREGO DO PROCESSO DOS DESLOCAMENTOS}

\section{C.1 DESCRIÇÃO DO SISTEMA}

\section{C.1.1 INTRODU ÇÃO}

Utilizando o Processo dos Deslocamentos, preparou-se um sistema de cálculo para microcomputadores em linguagem FORTRAN.

O sistema efetua a análise estática de cabos livremente suspensos, desde que as características geométricas iniciais - vão, coordenada no meio do vão, coordenadas dos apoios e área da seção transversal — as características físicas módulo de elasticidade do material - e as ações externas destes cabos sejam previamente especificadas.

\section{C.1.2 PROGRAMA PRINCIPAL}

O programa principal faz a leitura dos dados iniciais da estrutura, efetua cálculos preliminares e gera relatório destes dados lidos e calculados através da subrotina LEITURA. Efetua o cálculo do comprimento, dos cossenos diretores e da força axial iniciais de cada elemento e, do vetor com os esforços internos diferenciais iniciais da estrutura. Calcula a matriz de rigidez da estrutura através das subrotinas CALCRE e RIGIDEZ. Impõe as condições de contorno. Resolve o sistema de equações com a subrotina GAUSS. Calcula as novas coordenadas nodais. Calcula o comprimento, os cossenos diretores e força axial atuais de cada elemento - utilizando as subrotinas COMP, CALCCS, e CALCT. Calcula, também, os esforços internos nos nós e o desequilíbrio de cada nó. Imprime, ainda, relatório dos resultados da análise da estrutura no arquivo de saída de dados. 


\section{C.1.3 SUBROTINA LEITURA}

Esta subrotina lê os nomes dos arquivos de dados através do console do sistema. Estes arquivos são :

- ARQUIVO DE ENTRADA DE DADOS : arquivo old que contém os dados necessários ao cálculo do cabo ;

- ARQUIVO DE SAÍDA DE DADOS : arquivo new onde são escritos os dados de leitura e os resultados da análise.

Faz, também, a leitura dos dados iniciais da estrutura, efetua cálculos preliminares e do vetor das ações externas, e gera relatório dos dados lidos e calculados no arquivo de saída de dados.

\section{C.1.4 SUBROTINA CALCRE ( COSD, SEND, L, T, RE )}

Calcula a matriz de rigidez, $\mathrm{RE}$, do elemento com cossenos diretores, COSD e SEND, comprimento, L, e esforço axial, T.

\section{C.1.5 SUBROTINA RIGIDEZ ( K, RE )}

Faz a contribuição da matriz de rigidez, $\mathrm{RE}$, do elemento $\mathrm{K}$ na matriz de rigidez da estrutura.

\section{C.1.6 SUBROTINA CALCT ( $\mathrm{K}, \mathrm{L}, \mathrm{T}$ )}

Calcula o esforço axial, $\mathrm{T}$, do elemento $\mathrm{K}$ que possui comprimento atual igual a $\mathrm{L}$.

\section{C.1.7 SUBROTINA COMP ( K, L)}

Calcula o comprimento atual do elemento $\mathrm{K}, \mathrm{L}$. 


\section{C.1.8 SUBROTINA CALCCS ( $\mathrm{K}, \mathrm{L}, \mathrm{COSD}$, SEND )}

Calcula os cossenos diretores, COSD e SEND, do elemento $\mathrm{K}$ de comprimento igual a $\mathrm{L}$.

\section{C.1.9 SUBROTINA GAUSS ( NEQ )}

Calcula um sistema de equação com número de equações igual a NEQ.

\section{C.2 DESCRIÇÃO COMPLEMENTAR : O ARQUIVO DE ENTRADA DE DADOS}

$\mathrm{O}$ arquivo de entrada de dados deve ser preparado, previamente à execução do sistema, contendo os dados na forma sequencial.

Apresenta-se, a seguir, a estrutura deste arquivo sequencial :

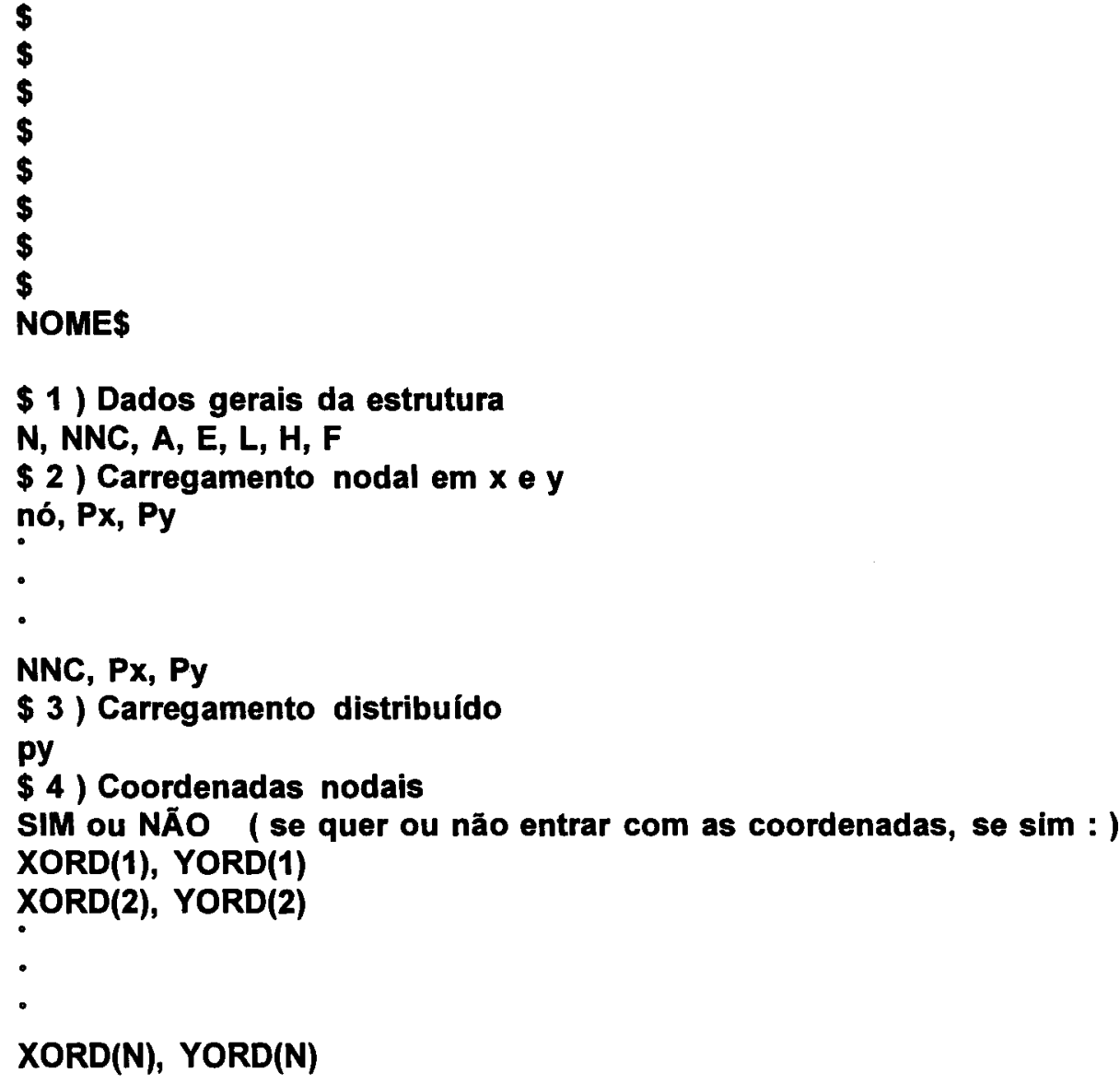

\$1) Dados gerais da estrutura

N, NNC, A, E, L, H, F

$\$ 2$ ) Carregamento nodal em $x$ e y nó, Px, Py

NNC, Px, Py

\$3) Carregamento distribuído

py

\$4) Coordenadas nodais

SIM ou NÄO ( se quer ou não entrar com as coordenadas, se sim : ) XORD(1), YORD(1)

XORD(2), YORD(2)

XORD(N), YORD(N) 
\$5) Condições de contorno

$\mathrm{RCH}(1), \operatorname{RCV}(1) \quad$ (para o nó A)

$\operatorname{RCH}(2), \operatorname{RCV}(2) \quad$ (para o nó $B$ )

O símbolo " \$ " quer dizer "linha para comentário".

A figura abaixo auxiliar a compreensão do arquivo de entrada de dados e as variáveis empregadas estão definidas no item C.3

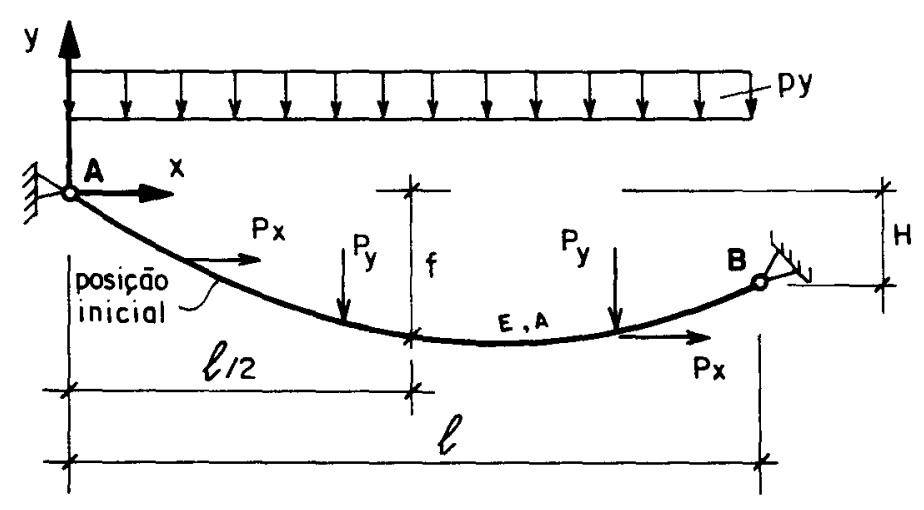

Figura C.2.1 - Ilustração das variáveis do arquivo de entrada de dados.

\section{C.3 - VARIÁVEIS UTILIZADAS NO SISTEMA}

\section{C.3.1 - PROGRAMA PRINCIPAL}

\section{Variáveis inteiras :}

$\mathrm{I}, \mathrm{J}$

contadores

$\mathrm{N} \quad$ : número de pontos nodais 


\section{Variáveis reais :}

$\begin{array}{lll}\text { A } & : \text { área da seção transversal do cabo } \\ \text { COSD,SEND } & : \text { cossenos diretores dos elementos } \\ \mathrm{E} & : \text { módulo de elasticidade do cabo } \\ \mathrm{FD} & : \text { vetor com esforços diferenciais internos } \\ \mathrm{FE} & : \text { vetor com as ações externas } \\ \mathrm{FI} & : \text { vetor com esforços internos (nodais) } \\ \mathrm{L} & : \text { vetor com os comprimentos dos elementos } \\ \mathrm{LO} & : \text { vetor com os comprimentos indeformados dos elementos } \\ \mathrm{R} & : \text { matriz de rigidez da estrutura } \\ \mathrm{RCH} & : \text { vetor com recalques horizontais } \\ \mathrm{RCV} & : \text { vetor com recalques verticais } \\ \mathrm{RE} & : \text { matriz de rigidez de um elemento } \\ \mathrm{V} & : \text { vetor com os deslocamentos nodais diferenciais } \\ \mathrm{XORD} & : \text { vetor com coordenadas nodais } \mathrm{x} \text { segundo sistema de } \\ & & \text { referência } \\ \mathrm{YORD} & \text { vetor com coordenadas nodais y segundo sistema de } \\ & & \text { referncia }\end{array}$

\section{C.3.2 - SUBROTINA LEITURA}

\begin{tabular}{ll}
\multicolumn{3}{c}{ Variáveis inteiras : } \\
MAXN & $:$ número máximo de pontos nodais \\
$\mathrm{N}$ & $:$ número de pontos nodais \\
$\mathrm{NCD}$ & $:$ número de carregamento distribuído \\
$\mathrm{NNC}$ & $:$ número de nós com carregamento
\end{tabular}

Variáveis reais :
A : área da seção transversal do cabo
E : módulo de elasticidade do cabo
F : flecha do cabo indeformado 
FE $\quad$ : vetor com as ações externas

$\mathrm{H} \quad$ : desnível

LO : vetor com o comprimento indeformado dos elementos

L : vão do cabo livremente suspenso

P : carregamento uniformemente distribuído segundo o vão

$\mathrm{RCH} \quad$ : vetor com recalques horizontais

$\mathrm{RCV} \quad$ : vetor com recalques verticais

XORD : vetor com coordenadas nodais $\mathrm{x}$ segundo sistema de referência

YORD : vetor com coordenadas nodais y segundo sistema de referência

\section{Variáveis do tipo caracter :}

AUX : variável auxiliar

NOME1 : arquivo old para leitura de dados

NOME2 : arquivo new para saída de dados

NOME\$ : nome do cabo

\section{C.3.3 - SUBROTINA CALCRE (COSD,SEND,L,T,RE)}

\section{Variáveis reais :}

A : área da seção transversal do cabo

AUX : variável auxiliar

COSD,SEND : cossenos diretores do elemento

E : módulo de elasticidade do material

L : comprimento do elemento

$\mathrm{T} \quad$ : esforço axial no elemento

$\mathrm{RE} \quad$ : matriz de rigidez do elemento 


\title{
C.3.4 - SUBROTINA RIGIDEZ (K,RE)
}

\author{
Variável inteira : \\ $\mathrm{K} \quad$ : número do elemento
}

\section{Variáveis reais :}

$\mathrm{R} \quad$ : matriz de rigidez da estrutura

$\mathrm{RE} \quad$ : matriz de rigidez do elemento

\section{C.3.5 - SUBROTINA CALCT (K,L,T)}

\section{Variável inteira :}

$\mathrm{K} \quad$ : número do elemento

\section{Variáveis reais :}

A : área da seção transversal do cabo

DELTAL : variação no comprimento do elemento

E : módulo de elasticidade

L : comprimento atual do elemento

$\mathrm{LO}(\mathrm{K}) \quad$ : comprimento indeformado do elemento

$\mathrm{T} \quad$ : esforço axial no elemento

\section{C.3.6 - SUBROTINA COMP(K,L)}

\section{Variável inteira :}

$\mathrm{K} \quad$ : número do elemento

\section{Variáveis reais :}

L : comprimento do elemento

VAL1 : variável auxiliar

VAL2 : variável auxiliar

XORD : vetor com as coordenadas nodais $\mathrm{x}$ segundo sistema de referência 
YORD

: vetor com as coordenadas nodais y segundo sistema de referência

\section{C.3.7 - SUBROTINA CALCCS ( K,L,COSD,SEND )}

Variável inteira :

$\mathrm{K}$

: número do elemento

\footnotetext{
Variáveis reais :

COSD,SEND : cossenos diretores do elemento

L : comprimento atual do elemento

XORD : vetor com as coordenadas nodais $\mathrm{x}$ segundo sistema de referência

YORD : vetor com as coordenadas nodais y segundo sistema de referência
}

C.4 - PROGRAMA FONTE

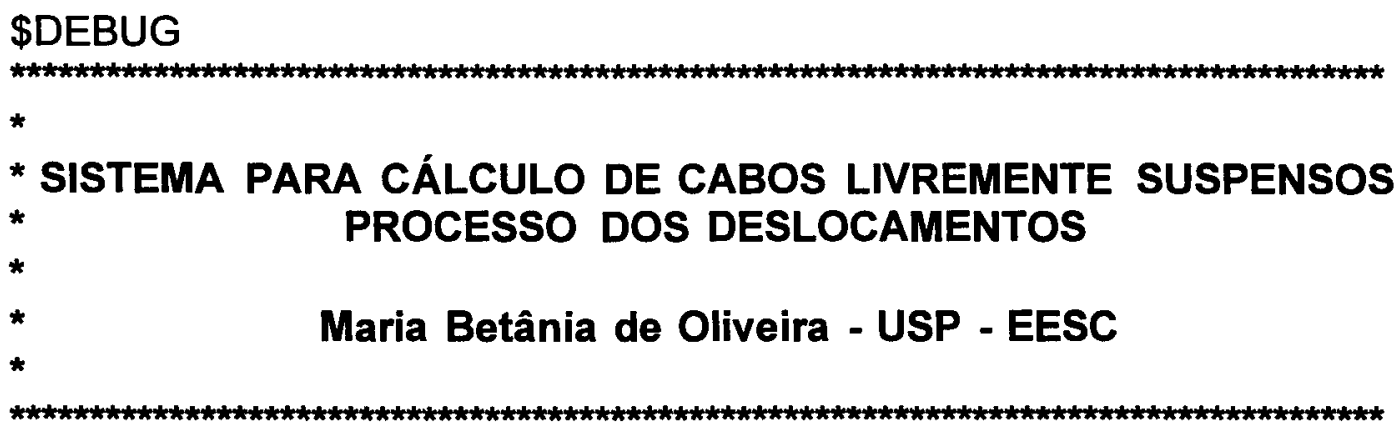

PROGRAMA PRINCIPAL

* a) Declaração das variáveis

INTEGER I,J,N,cont

DOUBLE PRECISION A,COSD(100), E,FD(200), FE(200), FI(200), * $L(100)$, $L O(100), R(200,200), R C H(2), R C V(2), R E(4,4)$, SEND(100),

* $T(100), V(200), X O R D(100), Y O R D(100)$ 
* b) Definição de área de memória

COMMON/BLOC1/A,E

COMMON/BLOC2/XORD,YORD

COMMON/BLOC3/LO

COMMON/BLOC4/FE

COMMON/BLOC5/N,RCH,RCV

COMMON/BLOC6/R

COMMON/BLOC7/FD,V

* c) Leitura dos dados iniciais, cálculos preliminares e relatório dos

* dados lidos e calculados

CALL LEITURA

* d) Cálculo do comprimento, dos cossenos diretores e da força axial inicial de cada elemento, e do vetor com os esforços internos diferenciais iniciais da estrutura

DO $1000, \mathrm{l}=1, \mathrm{~N}-1$

$\mathrm{L}(\mathrm{I})=\mathrm{LO}(\mathrm{I})$

CALL CALCCS (I,L(I),COSD(I),SEND(I))

$T(I)=A^{*} E / 1000$.

1000 CONTINUE

DO $1010,1=1,2^{*} \mathrm{~N}$

$\mathrm{FD}(\mathrm{I})=\mathrm{FE}(\mathrm{I})$

1010 CONTINUE

cont $=0$

555 DO $1015, \mathrm{l}=1,2^{*} \mathrm{~N}$

DO $1020, J=1,2 * N$

$R(I, J)=0$.

1020 CONTINUE

1015 CONTINUE

cont $=$ cont +1

* e) Cálculo da matriz de rigidez da estrutura

DO 1030, I=1,(N-1)

CALL CALCRE (COSD(I),SEND(I),L(I),T(I),RE)

CALL RIGIDEZ (I,RE)

1030 CONTINUE

* f) Imposição das condições de contorno

$R(1,1)=R(1,1)^{*} 10^{* *} 20$

$R(2,2)=R(2,2)^{*} 10^{* *} 20$ 
$\mathrm{R}\left(2^{*} \mathrm{~N}-1,2^{\star} \mathrm{N}-1\right)=\mathrm{R}\left(2^{\star} \mathrm{N}-1,2^{*} \mathrm{~N}-1\right)^{\star} 10^{* \star} 20$

$R\left(2^{\star} N, 2^{\star} N\right)=R\left(2^{\star} N, 2^{\star} N\right){ }^{*} 10^{\star \star} 20$

$\mathrm{FD}(1)=\mathrm{R}(1,1)^{*} \mathrm{RCH}(1)$

$F D(2)=R(2,2)^{\star} R C V(1)$

$\mathrm{FD}\left(2^{*} \mathrm{~N}-1\right)=\mathrm{R}\left(2^{*} \mathrm{~N}-1,2^{*} \mathrm{~N}-1\right)^{*} \mathrm{RCH}(2)$

$\mathrm{FD}\left(2^{*} \mathrm{~N}\right)=\mathrm{R}\left(2^{*} \mathrm{~N}, 2^{*} \mathrm{~N}\right){ }^{*} \mathrm{RCV}(2)$

* g) Resolução do sistema de equações

CALL GAUSS $\left(2^{*} \mathrm{~N}\right)$

* h) Cálculo das novas coordenadas nodais

$$
\begin{gathered}
\text { DO } 1040, \mathrm{I}=2, \mathrm{~N}-1 \\
\text { XORD }(\mathrm{I})=\text { XORD }(\mathrm{I})+\mathrm{V}\left(2^{*} \mid-1\right) \\
\text { YORD(I) }=\text { YORD }(\mathrm{I})+\mathrm{V}\left(2^{*} \mathrm{I}\right) \\
1040 \text { CONTINUE }
\end{gathered}
$$

* i) Cálculo do comprimento, dos cossenos diretores, da força axial

* atuais de cada elemento

DO $1050, \mathrm{I}=1, \mathrm{~N}-1$

CALL COMP(I,L(I))

CALL CALCCS (I,L(I),COSD(I),SEND(I))

CALL CALCT $(\mathrm{l}, \mathrm{L}(\mathrm{I}), \mathrm{T}(\mathrm{I}))$

1050 CONTINUE

* j) Cálculo dos esforços internos (nodais)

$\mathrm{Fl}(1)=-\mathrm{T}(1)^{\star} \operatorname{CosD}(1)$

$\mathrm{FI}(2)=-\mathrm{T}(1)^{\star} \mathrm{SEND}(1)$

$\mathrm{FI}\left(2^{*} \mathrm{~N}-1\right)=\mathrm{T}(\mathrm{N}-1)^{*} \operatorname{CoSD}(\mathrm{N}-1)$

$\mathrm{FI}\left(2^{*} \mathrm{~N}\right)=\mathrm{T}(\mathrm{N}-1)^{*} \operatorname{SEND}(\mathrm{N}-1)$

DO $1060, \mathrm{l}=2, \mathrm{~N}-1$

$\mathrm{FI}\left(2^{*} \mathrm{I}-1\right)=\mathrm{T}(\mathrm{I}-1)^{*} \operatorname{Cos} \mathrm{C}(\mathrm{I}-1)-\mathrm{T}(\mathrm{I})^{*} \operatorname{Cos} \mathrm{C}(\mathrm{I})$

$\mathrm{FI}\left(2^{*} \mathrm{I}\right)=\mathrm{T}(\mathrm{I}-1)^{*} \mathrm{SEND}(\mathrm{I}-1)-\mathrm{T}(\mathrm{I})^{*} \mathrm{SEND}(\mathrm{I})$

1060 CONTINUE

do $1061, i=1,2 * \mathrm{~N}$

write $\left({ }^{*},{ }^{*}\right) \mathrm{Fl}(\mathrm{i})$

1061 continue

* l) Cálculo do desequilíbrio - cálculo do novo vetor de esforços

* internos diferenciais

DO $1070, l=1,2^{*} \mathrm{~N}$

$\mathrm{FD}(\mathrm{I})=\mathrm{FE}(\mathrm{I})-\mathrm{FI}(\mathrm{I})$ 
1070 CONTINUE

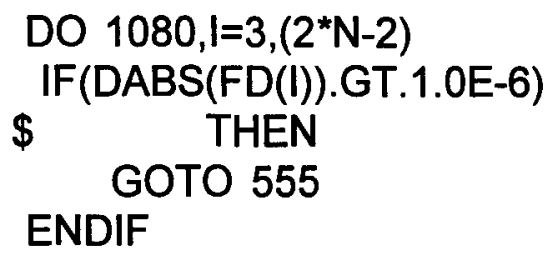

1080 CONTINUE

* m) Impressão do relatório

WRITE $(3,10)$

DO $1090, \mathrm{~J}=1, \mathrm{~N}$

WRITE $(3,15)$ J,XORD(J),YORD(J)

1090 CONTINUE

WRITE $(3,20)$

DO $1100, \mathrm{~J}=1, \mathrm{~N}-1,2$

WRITE $(3,25) \mathrm{J}, \mathrm{T}(\mathrm{J}),(\mathrm{J}+1), \mathrm{T}(\mathrm{J}+1)$

1100 CONTINUE

write $\left({ }^{\star}, 1\right)$

1 format( $/ I$, ' Numero de interacoes : ',l)

write $\left({ }^{*},{ }^{*}\right)$ cont

10 FORMAT(II,6X,'5)POSICAO FINAL DE EQUILIBRIO'I, X,' No' 17X,'X', *17 X, ' $Y^{\prime}$, , / )

15 FORMAT(6X,I3,3X,F15.6,3X,F15.6)

20 FORMAT( $(I, 6 X, ' 6)$ ESFORCOS FINAIS NOS ELEMENTOS ',l,6X, * 'Elemento', 17X,'T',3X,'Elemento',17X,'T',I)

25 FORMAT(9X,I3,5X,F15.5,6X,I3,5X,F15.5)

STOP

END

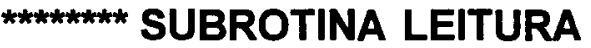

a) Declaração das variáveis

INTEGER I,K(10),MAXN,N,NNC

DOUBLE PRECISION A,E,F,FE(200),L,LO(100),P,PH,PN,RCH(2),

${ }^{*} \mathrm{RCV}(2), \mathrm{X}, \mathrm{XORD}(100), \mathrm{YORD}(100)$

CHARACTER 7 NOME1,NOME2

CHARACTER*80 AUX,NOME\$

* b) Definição da área de memória

COMMON/BLOC1/A,E 
COMMON/BLOC2/XORD,YORD

COMMON/BLOC3/LO

COMMON/BLOC4/FE

COMMON/BLOC5/N,RCH,RCV

* d) Definição do limite da variável $\mathbf{N}$

$\operatorname{MAXN}=100$

* e) Leitura dos dados, cálculos preliminares e relatório

* e.1) Leitura dos dados

WRITE $(*, 1)$

WRITE $\left({ }^{*}, 2\right)$

WRITE $\left({ }^{*}, 5\right)$

READ $(*, 6)$ NOME1

WRITE $(*, 7)$

READ $(*, 6)$ NOME2

OPEN (2,FILE=NOME1,STATUS='OLD')

OPEN (3,FILE=NOME2,STATUS='NEW')

DO $1000, I=1,9$

$\operatorname{READ}(2,10)$ AUX

1000 CONTINUE

READ $(2,10)$ NOME\$

READ $(2,10)$ AUX

$\operatorname{READ}\left(2,{ }^{*}\right) \mathrm{N}, \mathrm{NNC}, \mathrm{A}, \mathrm{E}, \mathrm{L}, \mathrm{H}, \mathrm{F}$

1 FORMAT(40(l))

2 FORMAT(81('*'),I,1X,'*', 15X,'SISTEMA PARA CALCULO DO CABO *LIVREMENTE SUSPENSO',15X,'*',I,1X,'*',21X,'EMPREG0 DOPRO ${ }^{*}$ CESSO DOS DESLOCAMENTOS',21X,"*',l,1X,"*',20X,'Maria Betania

* de Oliveira - USP - EESC',20X,"*',I,81('*'),/)

5 FORMAT(2(l),6X,'NOME DO ARQUIVO DE ENTRADA DE DADOS * (ATE 7 DIGITOS )?)', ()

6 FORMAT(A7)

7 FORMAT(/I,6X,'NOME DO ARQUIVO DE SAIDA DE DADOS(ATE 7 * DIGITOS)? ', I)

10 FORMAT(A80) 
d.1.2) Teste de consistência

IF(N.GT.MAXN)

$\$$ THEN

WRITE $(*, 15)$ MAXN

$\operatorname{READ}\left({ }^{*}, *\right)$

ENDIF

15 FORMAT(II,6X,'ERRO - ULTRAPASSOU QUATIDADE MAXIMA DE * NOS QUE E',I3)

* d.1.3) Impressão dos dados gerais

WRITE $(3,6)$ NOME2

WRITE $(3,12)$

WRITE $(3,20)$ NOME $\$, N, N N C, A, E, L, H, F$

12 FORMAT(80(' * '),l,' * ',78X,' * ',l,' *',15X,'SISTEMA PARA CALCULO *DO CABO LIVREMENTE SUSPENSO',15X,'* ',l,'* ',78X,'*',l,', ', 21X * , 'EMPREGO DO PROCESSO DOS DESLOCAMENTOS',21X,' " ',l,' * *',78X,' * ',l,"* ',20X,'Maria Betania de Oliveira - USP - EESC',20X,'

* * ',l,' * ',78X,' * ',l,80(' * '))

20 FORMAT(II,6X,'NOME DA ESTRUTURA : ',A80, /I,6X,'1) DADOS GE * RAIS DA ESTRUTURA',I,6X,'Numero de pontos nodais',9X,': ',12X * $13, I, 6 \mathrm{X}$, 'Numero de nos carregados ',8X, ' : ',1 3X,12,I,6X,'Area da * secao transversal', 7X,' :', F15.10,l, 6X,'Modulo de elasticida de', *10X, ': ',F15.2,I,6X,'Vao',29X,': ', F15.3 ,I,6X,'Desnivel ', 24X ,': ', F15

* $3, I, 6 \mathrm{X}$,'Flecha do cabo indeformado',5X,' : ',F15.3)

* d.2) Dados sobre o carregamento

* d.2.1) Zerando FE

DO $1010, I=1,2^{*} \mathrm{~N}$

$\mathrm{FE}(\mathrm{I})=0$.

1010 CONTINUE

* d.2.2) Carregamento concentrado

$\operatorname{READ}(2,10)$ AUX

DO 1020,I=1, NNC

$\operatorname{READ}\left(2,{ }^{*}\right) \mathrm{K}(\mathrm{I}), \mathrm{FE}\left(2^{*} \mathrm{~K}(\mathrm{I})-1\right), \mathrm{FE}\left(2^{\star} \mathrm{K}(\mathrm{I})\right)$

1020 CONTINUE 
* d.2.3) Carregamento distribuido no vão

$\operatorname{READ}(2,10)$ AUX

$\operatorname{READ}(2, *) P$

* d.2.4) Impressão dos dados

WRITE $(3,25)$

DO $1040, \mathrm{l}=1, \mathrm{NNC}$

WRITE $(3,27) \mathrm{K}(\mathrm{I}), \mathrm{FE}\left(2^{*} \mathrm{~K}(\mathrm{I})-1\right), \mathrm{FE}\left(2^{*} \mathrm{~K}(\mathrm{I})\right)$

1040 CONTINUE

WRITE $(3,28)$

WRITE $(3,29)$ P

25 FORMAT(II,6X,'2)DADOS DO CARREGAMENTO',I,6X,'Carregamento

* Concentrado : No',9X,'Px',9X,'Py')

27 FORMAT(33X,13,3X,F8.2,3X,F8.2)

28 FORMAT(6X,'Carregamento distribuido :',13X,'py')

29 FORMAT(36X,F11.3)

* d.3) Coordenadas nodais iniciais

$\operatorname{READ}(2,10)$ AUX $\operatorname{READ}(2,10)$ AUX

IF(AUX.EQ.'SIM')

$\$$ THEN

DO $1060, I=1, N$

$\operatorname{READ}(2, *)$ XORD(I),YORD(I)

1060 CONTINUE

ELSE

$\mathrm{PH}=4^{*}\left(-2^{*} \mathrm{~F}+\mathrm{H}\right) /\left(\mathrm{L}^{\star \star} 2\right)$

$\mathrm{X}=\mathrm{L}(\mathrm{N}-1)$

$\mathrm{XORD}(1)=0$.

$\operatorname{YORD}(1)=0$.

DO $1065, I=2, N$

$X O R D(I)=X O R D(I-1)+X$

1065 CONTINUE

YORD $(I)=+\mathrm{PH}^{\star}\left(\mathrm{XORD}(\mathrm{I})^{\star \star} 2\right) / 2+\left(-\mathrm{PH}^{\star} \mathrm{L} / 2+\mathrm{H} / \mathrm{L}\right)^{\star} \mathrm{XORD}(\mathrm{I})$

ENDIF

WRITE $(3,35)$

DO $1070, I=1, N$

WRITE $(3,36)$ I,XORD(I),YORD(I)

1070 CONTINUE 
35 FORMAT(II,6X,3) COORDENADAS NODAIS INICIAIS NO SISTEMA

* DE REFERENCIA',I,7X,'No',10X,'X',10X,'Y')

36 FORMAT(6X,I3,3X,F8.2,3X,F8.2)

* d.4) Cálculo do coprimento inicial dos elementos

$$
\begin{aligned}
& \text { DO } 1075, \mathrm{I}=1, \mathrm{~N}-1 \\
& \text { CALL COMP(I,LO(I)) } \\
& 1075 \text { CONTINUE }
\end{aligned}
$$

* d.5) Cálculo de FE inicial

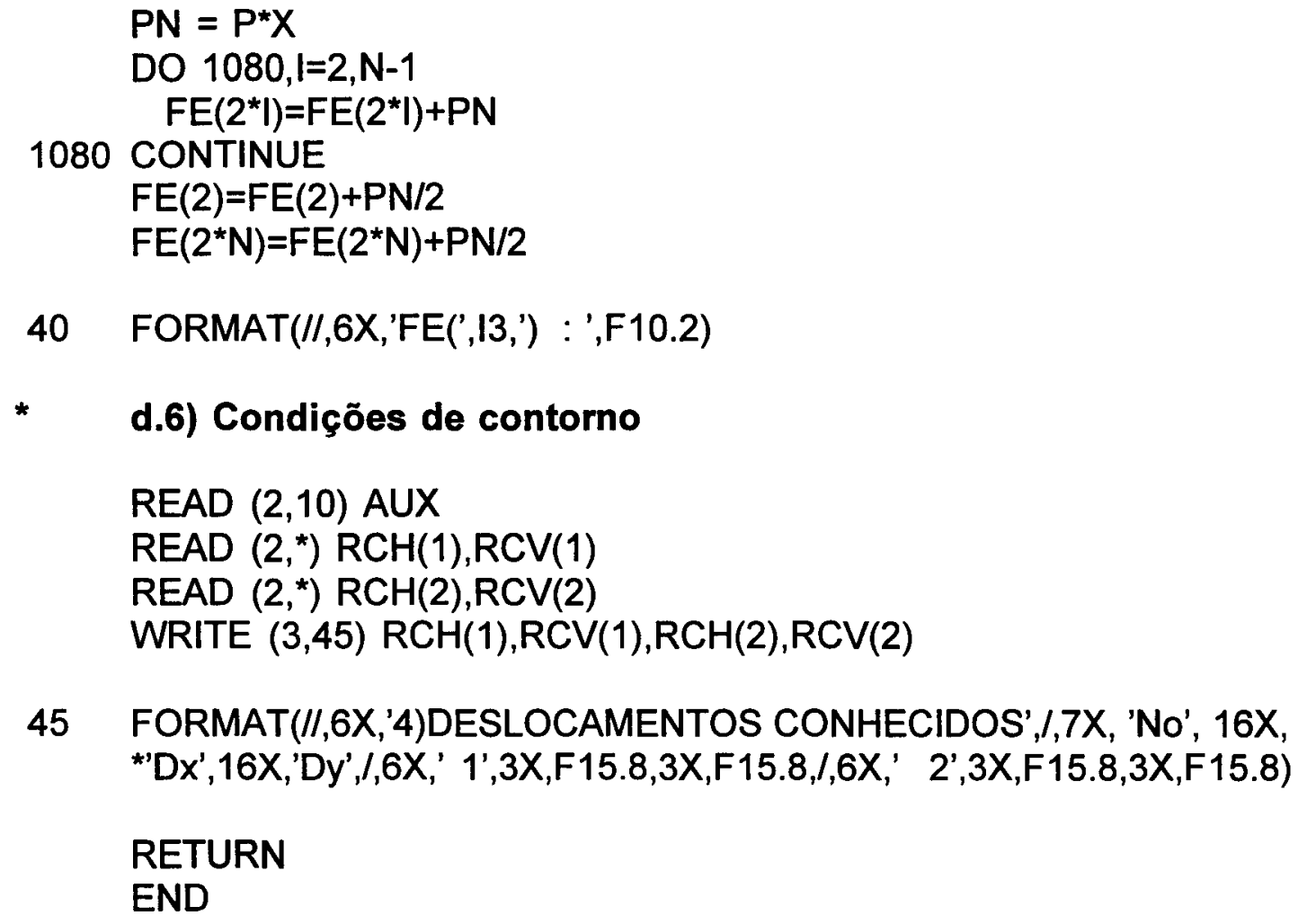

40 FORMAT(//,6X,'FE(',13,') : ',F10.2)

* d.6) Condições de contorno

$\operatorname{READ}(2,10)$ AUX

$\operatorname{READ}\left(2,{ }^{*}\right) \mathrm{RCH}(1), \mathrm{RCV}(1)$

$\operatorname{READ}\left(2,{ }^{\star}\right) \operatorname{RCH}(2), \operatorname{RCV}(2)$

WRITE $(3,45) \operatorname{RCH}(1), \operatorname{RCV}(1), \operatorname{RCH}(2), \operatorname{RCV}(2)$

45 FORMAT(II,6X,'4)DESLOCAMENTOS CONHECIDOS',/,7X, 'No', 16X, *'Dx',16X,'Dy',/,6X,' 1',3X,F15.8,3X,F15.8,/,6X,' 2',3X,F15.8,3X,F15.8)

RETURN

END

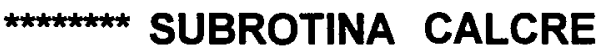

SUBROUTINE CALCRE (COSD,SEND,L,T,RE)

a) Declaração das variáveis

DOUBLE PRECISION A,AUX,COSD,E,L,T,RE(4,4),SEND 
* b) Definição de área de memória

COMMON/BLOC1/A,E

* c) Cálculo da matriz de rigidez do elemento

$$
\begin{aligned}
& A U X=E^{\star} A / L \\
& \operatorname{RE}(1,1)=T / L+A U X^{*} \operatorname{COSD}^{* *} 2 \\
& \operatorname{RE}(1,2)=\text { AUX }^{*} \operatorname{COSD}^{*} \text { SEND } \\
& \operatorname{RE}(1,3)=-T / L-A U X^{\star}{ }^{2} O S D^{\star \star} 2 \\
& \operatorname{RE}(1,4)=-A U X^{*} S E N D^{*} \text { COSD } \\
& \operatorname{RE}(2,2)=T / L+A U X^{\star} S E N D^{\star *} 2 \\
& \operatorname{RE}(2,3)=- \text { AUX }^{*} \text { SEND*COSD } \\
& \operatorname{RE}(2,4)=-T / L-A U X * S E N D^{* *} 2 \\
& \operatorname{RE}(3,3)=T / L+A U X^{*} \operatorname{COSD}^{\star \star} 2 \\
& \operatorname{RE}(3,4)=\text { AUX }^{*} \text { SEND }{ }^{*} \text { COSD } \\
& \operatorname{RE}(4,4)=T / L+A U X{ }^{*} S E N D^{\star *} 2 \\
& \operatorname{RE}(2,1)=\operatorname{RE}(1,2) \\
& \operatorname{RE}(3,1)=\operatorname{RE}(1,3) \\
& \operatorname{RE}(3,2)=\operatorname{RE}(2,3) \\
& \operatorname{RE}(4,1)=\operatorname{RE}(1,4) \\
& \operatorname{RE}(4,2)=\operatorname{RE}(2,4) \\
& \operatorname{RE}(4,3)=\operatorname{RE}(3,4) \\
& \text { RETURN } \\
& \text { END }
\end{aligned}
$$

SUBROUTINE RIGIDEZ (K,RE)

* a) Declaração das variáveis

INTEGER $\mathrm{K}$

DOUBLE PRECISION R(200,200),RE(4,4)

* b) Definição de área de memória

COMMON/BLOC6/R

* c) Contribuição do elemento na matriz de rigidez da estrutura

$\mathrm{R}\left(2^{*} \mathrm{~K}-1,2^{*} \mathrm{~K}-1\right)=\mathrm{R}\left(2^{*} \mathrm{~K}-1,2^{*} \mathrm{~K}-1\right)+\mathrm{RE}(1,1)$

$\mathrm{R}\left(2^{*} \mathrm{~K}-1,2^{\star} \mathrm{K}\right)=\mathrm{R}\left(2^{\star} \mathrm{K}-1,2^{*} \mathrm{~K}\right)+\mathrm{RE}(1,2)$

$R\left(2^{*} K-1,2^{*} K+1\right)=R\left(2^{*} K-1,2^{*} K+1\right)+R E(1,3)$ 
$R\left(2^{*} \mathrm{~K}-1,2^{*} \mathrm{~K}+2\right)=\mathrm{R}\left(2^{*} \mathrm{~K}-1,2^{*} \mathrm{~K}+2\right)+\mathrm{RE}(1,4)$

$\mathrm{R}\left(2^{*} \mathrm{~K}, 2^{*} \mathrm{~K}-1\right)=\mathrm{R}\left(2^{*} \mathrm{~K}, 2^{*} \mathrm{~K}-1\right)+\mathrm{RE}(2,1)$

$R\left(2^{*} K, 2^{*} K\right)=R\left(2^{*} K, 2^{*} K\right)+R E(2,2)$

$R\left(2^{*} K, 2^{*} K+1\right)=R\left(2^{*} K, 2^{*} K+1\right)+R E(2,3)$

$R\left(2^{*} K, 2^{*} K+2\right)=R\left(2^{*} K, 2^{*} K+2\right)+R E(2,4)$

$R\left(2^{\star} \mathrm{K}+1,2^{\star} \mathrm{K}-1\right)=\mathrm{R}\left(2^{\star} \mathrm{K}+1,2^{\star} \mathrm{K}-1\right)+\mathrm{RE}(3,1)$

$R\left(2^{*} K+1,2^{*} K\right)=R\left(2^{*} K+1,2^{*} K\right)+R E(3,2)$

$R\left(2^{*} K+1,2^{\star} K+1\right)=R\left(2^{*} K+1,2^{*} K+1\right)+R E(3,3)$

$R\left(2^{\star} K+1,2^{\star} K+2\right)=R\left(2^{\star} K+1,2^{*} K+2\right)+R E(3,4)$

$\mathrm{R}\left(2^{\star} \mathrm{K}+2,2^{\star} \mathrm{K}-1\right)=\mathrm{R}\left(2^{\star} \mathrm{K}+2,2^{\star} \mathrm{K}-1\right)+\mathrm{RE}(4,1)$

$R\left(2^{*} K+2,2^{*} K\right)=R\left(2^{*} K+2,2^{*} K\right)+R E(4,2)$

$R\left(2^{*} K+2,2^{\star} K+1\right)=R\left(2^{\star} K+2,2^{*} K+1\right)+R E(4,3)$

$R\left(2^{\star} K+2,2^{\star} K+2\right)=R\left(2^{\star} K+2,2^{\star} K+2\right)+R E(4,4)$

RETURN

END

SUBROTINA CALCT

SUBROUTINE CALCT (K,L,T)

* a) Declaração das variáveis

INTEGER K

DOUBLE PRECISION A,DELTAL,E,L,LO(100),T

* b) Definição de área de memória

COMMON/BLOC1/A,E

COMMON/BLOC3/LO

c) Cálculo do esforço axial no elemento

DELTAL $=L-L O(K)$

$T=E^{*} A^{*} D E L T A L / L O(K)$

RETURN

END 


\section{SUBROUTINE COMP ( $K, L$ )}

* a) Declaração das variáveis

INTEGER $K$

DOUBLE PRECISION L,VAL1,VAL2,XORD(100),YORD(100)

* b) Definição de área de memória

COMMON/BLOC2/XORD,YORD

* c) Cálculo do comprimento do elemento

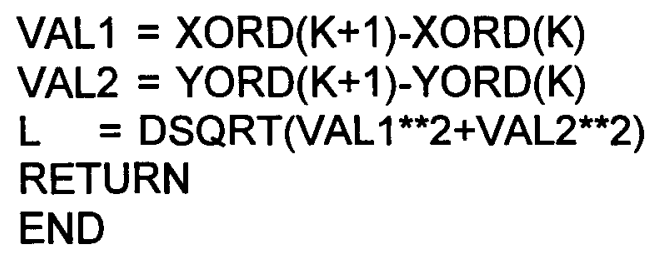

****** SUBROTINA CALCCS

SUBROUTINE CALCCS ( K,L,COSD,SEND )

* a) Declaração das variáveis

INTEGER $K$

DOUBLE PRECISION COSD,L,SEND,XORD(100),YORD(100)

* b) Definição de área de memória

COMMON/BLOC2/XORD,YORD

* c) Cálculo dos cossenos diretores

$\operatorname{COSD}=(\mathrm{XORD}(\mathrm{K}+1)-\mathrm{XORD}(\mathrm{K})) / \mathrm{L}$

SEND $=(Y O R D(K+1)-Y O R D(K)) / L$

RETURN

END 


\section{SUBROUTINE GAUSS(NEQ)}

* a) Declaração das variáveis

DOUBLE PRECISION C(200),A(200,200),R,X(200)

INTEGER K,I,J,NEQ

* b) Definição de área de memória

COMMON/BLOC6/ A

COMMON/BLOC7/ C,X

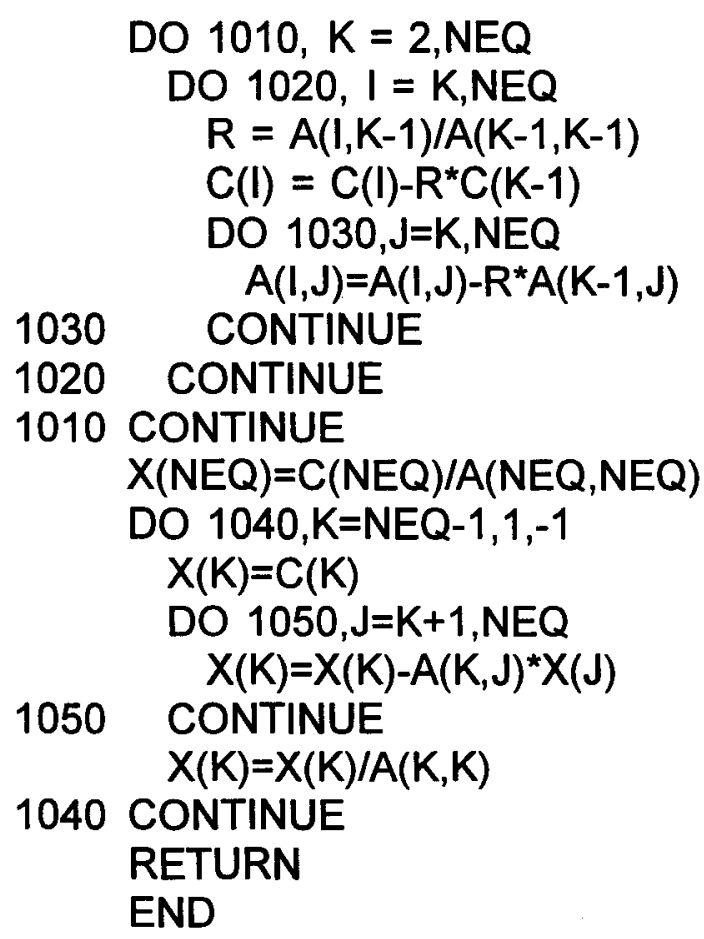




\section{APÊNDICE D}

\section{RESULTADOS DA EXPERIMENTAÇÃO NUMÉRICA}

Tabela D.1 - Símbolos utilizados no apêndice D

\begin{tabular}{|c|l|}
\hline SÍMBOLO & \\
\hline $\mathrm{A}$ & área metálica da seção transversal do cabo \\
\hline $\mathrm{E}$ & módulo de elasticidade do cabo \\
\hline erro & erro cometido com o PA em relação ao PD \\
\hline $\mathrm{E} 1$ & erro cometido na primeira aproximação \\
\hline E2 & erro cometido na segunda aproximação \\
\hline E3 & erro cometido na terceira aproximação \\
\hline E4 & erro cometido na quarta aproximação \\
\hline $\mathrm{f}$ & coordenada inicial y no meio do vão \\
\hline $\mathrm{h}$ & desnível entre os apoios \\
\hline$\overline{\mathrm{H}}$ & esforço horizontal final na seção do cabo \\
\hline$\ell$ & vão do cabo \\
\hline Nome do & nome do cabo que possui determinadas características geométricas e \\
Cabo & fisicas e carregamento \\
\hline Número de & número de segmentos lineares utilizados para o cálculo do cabo pelo \\
segmentos & Processo dos Deslocamentos \\
\hline$\overline{\mathrm{p}}$ & carregamento final uniformemente distribuído ao longo de todo o vão \\
\hline PA & resultados obtidos com o Processo Analítico \\
\hline PD & resultados obtidos com o Processo dos Deslocamentos \\
\hline Tpd & esforço de tração empregado no pré-dimensionamento do cabo \\
\hline $\bar{T}$ máx & máximo esforço final de tração no cabo \\
\hline $\bar{y}$ máx & máxima coordenada y final do cabo \\
\hline
\end{tabular}


TABELA D.2 - Comparação entre os resultados do Processo dos Deslocamentos com o aumento do número de segmentos - cabo com $\mathrm{E}=120 \mathrm{E}+9 \mathrm{~N} / \mathrm{m}^{2}$, $\mathrm{A}=258,06 \mathrm{~mm}^{2}, \mathrm{f}=4 \mathrm{~m}, \ell=40 \mathrm{~m}, \mathrm{~h}=0$ e $\overline{\mathrm{p}}=5000 \mathrm{~N} / \mathrm{m}$.

\begin{tabular}{|c|r|r|r|r|r|r|}
\hline Número de segmentos & $\overline{\mathbf{H}}(\mathbf{N})$ & erro \% & $\overline{\mathbf{T}}$ máx $(\mathbf{N})$ & \multicolumn{1}{c|}{ erro \% } & $\overline{\mathbf{Y}}$ máx $(\mathbf{m})$ & \multicolumn{1}{c|}{ erro \% } \\
\hline 10 & 219024 & & 236583 & & 4,555 & \\
20 & 218986 & $1,74 \mathrm{E}-02$ & 238652 & $-8,67 \mathrm{E}-01$ & 4,552 & $6,59 \mathrm{E}-02$ \\
30 & 218978 & $3,65 \mathrm{E}-03$ & 239342 & $-2,88 \mathrm{E}-01$ & 4,552 & $0,00 \mathrm{E}+00$ \\
40 & 218975 & $1,37 \mathrm{E}-03$ & 239688 & $-1,44 \mathrm{E}-01$ & 4,551 & $2,20 \mathrm{E}-02$ \\
50 & 218974 & $4,57 \mathrm{E}-04$ & 239895 & $-8,63 \mathrm{E}-02$ & 4,551 & $0,00 \mathrm{E}+00$ \\
60 & 218974 & $0,00 \mathrm{E}+00$ & 240034 & $-5,79 \mathrm{E}-02$ & 4,551 & $0,00 \mathrm{E}+00$ \\
70 & 218973 & $4,57 \mathrm{E}-04$ & 240133 & $-4,12 \mathrm{E}-02$ & 4,551 & $0,00 \mathrm{E}+00$ \\
80 & 218973 & $0,00 \mathrm{E}+00$ & 240207 & $-3,08 \mathrm{E}-02$ & 4,551 & $0,00 \mathrm{E}+00$ \\
90 & 218973 & $0,00 \mathrm{E}+00$ & 240264 & $-2,37 \mathrm{E}-02$ & 4,551 & $0,00 \mathrm{E}+00$ \\
\hline
\end{tabular}

TABELA D.3 - Comparação entre os resultados do Processo dos Deslocamentos com o aumento do número de segmentos - cabo com $\mathrm{E}=120 \mathrm{E}+9 \mathrm{~N} / \mathrm{m}^{2}$, $\mathrm{A}=258,06 \mathrm{~mm}^{2}, \mathrm{f}=6 \mathrm{~m}, \ell=60 \mathrm{~m}, \mathrm{~h}=10 \mathrm{~m}$ e $\overline{\mathrm{p}}=5000 \mathrm{~N} / \mathrm{m}$.

\begin{tabular}{|c|r|c|r|r|r|r|}
\hline Número de segmentos & $\overline{\mathbf{H}}(\mathbf{N})$ & erro \% & $\overline{\mathbf{T}}$ máx (N) & erro \% & $\overline{\mathbf{Y}}$ máx(m) & \multicolumn{1}{c|}{ erro \% } \\
\hline 10 & 462443 & & 508838 & & 10,583 & \\
20 & 462772 & $-7,11 \mathrm{E}-02$ & 513066 & $-8,24 \mathrm{E}-01$ & 11,101 & $-4,67 \mathrm{E}+00$ \\
30 & 462972 & $-4,32 \mathrm{E}-02$ & 514341 & $-2,48 \mathrm{E}-01$ & 11,102 & $-9,01 \mathrm{E}-03$ \\
40 & 463022 & $-1,08 \mathrm{E}-02$ & 514953 & $-1,19 \mathrm{E}-01$ & 11,100 & $1,80 \mathrm{E}-02$ \\
50 & 463057 & $-7,56 \mathrm{E}-03$ & 515312 & $-6,97 \mathrm{E}-02$ & 11,102 & $-1,80 \mathrm{E}-02$ \\
60 & 463073 & $-3,46 \mathrm{E}-03$ & 515548 & $-4,58 \mathrm{E}-02$ & 11,101 & $9,01 \mathrm{E}-03$ \\
70 & 463081 & $-1,73 \mathrm{E}-03$ & 515716 & $-3,26 \mathrm{E}-02$ & 11,101 & $0,00 \mathrm{E}+00$ \\
80 & 463090 & $-1,94 \mathrm{E}-03$ & 515840 & $-2,40 \mathrm{E}-02$ & 11,101 & $0,00 \mathrm{E}+00$ \\
90 & 463093 & $-6,48 \mathrm{E}-04$ & 515936 & $-1,86 \mathrm{E}-02$ & 11,101 & $0,00 \mathrm{E}+00$ \\
\hline
\end{tabular}


TABELA D.4- Comparação entre os resultados do Processo dos Deslocamentos dos cabos empregados na TABELA D.5.

\begin{tabular}{|c|c|c|c|c|c|c|c|c|}
\hline $\begin{array}{c}\text { Nome } \\
\text { do cabo }\end{array}$ & $\begin{array}{l}\text { Número de } \\
\text { segmentos }\end{array}$ & $\begin{array}{c}A \\
\left(\mathrm{~mm}^{2}\right)\end{array}$ & $\begin{array}{c}\overline{\bar{H}} \\
(\mathbf{N})\end{array}$ & erro \% & $\begin{array}{c}\mathbf{T} \text { máx } \\
(\mathrm{N})\end{array}$ & erro \% & $\begin{array}{c}\overline{\mathbf{Y}} \text { máx } \\
\text { (m) }\end{array}$ & erro \% \\
\hline \multirow{3}{*}{ Al } & 80 & \multirow{3}{*}{25,22} & 375429 & \multirow{3}{*}{$\begin{array}{l}0,001065459 \\
0,000799101\end{array}$} & 544929 & \multirow{3}{*}{$\begin{array}{r}-0,074083175 \\
-0,048387363\end{array}$} & 20,148 & \multirow{3}{*}{$\begin{array}{l}0 \\
0\end{array}$} \\
\hline & 90 & & 375425 & & 545333 & & 20,148 & \\
\hline & 98 & & 375422 & & 545597 & & 20,148 & \\
\hline \multirow{3}{*}{ A2 } & 80 & \multirow{3}{*}{81,68} & 581755 & & 703164 & \multirow{3}{*}{$\begin{array}{r}-0,044919543 \\
-0,029274421\end{array}$} & 13,369 & \multirow{3}{*}{$\begin{array}{l}0 \\
0\end{array}$} \\
\hline & 90 & & 581755 & 0 & 703480 & & 13,369 & \\
\hline & 98 & & 581755 & 0 & 703686 & & 13,369 & \\
\hline \multirow{3}{*}{ A3 } & 80 & \multirow{3}{*}{145,16} & 706605 & & 809500 & \multirow{3}{*}{$\begin{array}{r}-0,0340835 \\
-0,022223429\end{array}$} & 11,103 & \multirow{3}{*}{$\begin{array}{l}0 \\
0\end{array}$} \\
\hline & 90 & & 706607 & $-0,000283043$ & 809776 & & 11,103 & \\
\hline & 98 & & 706607 & 0 & 809956 & & 11,103 & \\
\hline \multirow{3}{*}{ A4 } & 70 & \multirow{3}{*}{403,23} & 968214 & & 1045403 & \multirow{3}{*}{$\begin{array}{r}-0,026776757 \\
-0,020747681\end{array}$} & 8,182 & \multirow{3}{*}{$\begin{array}{l}0 \\
0\end{array}$} \\
\hline & 80 & & 968221 & $-0,000722975$ & 1045683 & & 8,182 & \\
\hline & 90 & & 968226 & $-0,000516408$ & 1045900 & & 8,182 & \\
\hline \multirow{3}{*}{ A5 } & 70 & \multirow{3}{*}{790,32} & 1162580 & & 1227608 & \multirow{3}{*}{$\begin{array}{l}-0,019627821 \\
-0,015227567\end{array}$} & 6,840 & \multirow{3}{*}{$\begin{array}{l}0 \\
0\end{array}$} \\
\hline & 80 & & 1162589 & $-0,000774134$ & 1227849 & & 6,840 & \\
\hline & 90 & & 1162595 & $-0,000516087$ & 1228036 & & 6,840 & \\
\hline \multirow{3}{*}{ A6 } & 70 & \multirow{3}{*}{1165,54} & $\overline{1278166}$ & & 1337586 & \multirow{3}{*}{$\begin{array}{r}-0,016669046 \\
-0,01285519\end{array}$} & 6,231 & \multirow{3}{*}{$\begin{array}{l}0 \\
0\end{array}$} \\
\hline & 80 & & 1278176 & $-0,000782365$ & 1337809 & & 6,231 & \\
\hline & 90 & & 1278182 & $-0,000469417$ & 1337981 & & 6,231 & \\
\hline \multirow{3}{*}{ A7 } & 70 & \multirow{3}{*}{1612,90} & 1373958 & & 1429402 & \multirow{3}{*}{$\begin{array}{r}-0,014619361 \\
-0,01133047\end{array}$} & 5,802 & \multirow{3}{*}{$\begin{array}{l}0 \\
0\end{array}$} \\
\hline & 80 & & 1373968 & $-0,000727819$ & 1429611 & & 5,802 & \\
\hline & 90 & & 1373975 & $-0,000509471$ & 1429773 & & 5,802 & \\
\hline
\end{tabular}


TABELA D. 5 - Comparação entre os resultados do Processo Analítico e Processo dos deslocamento - cabos com $\overline{\mathbf{p}}=10000 \mathrm{~N} / \mathrm{m}$, $\mathrm{E}=120 \mathrm{E}+9 \mathrm{~N} / \mathrm{m}^{2}, \mathrm{f}=4 \mathrm{~m}, \mathrm{~h}=0 \mathrm{e} \ell=80 \mathrm{~m}$

\begin{tabular}{|c|c|c|c|c|c|c|c|c|c|c|}
\hline \multirow{2}{*}{$\begin{array}{l}\text { Nome } \\
\text { do cabo }\end{array}$} & \multirow{2}{*}{$\begin{array}{c}\text { Area } \\
\left(\mathrm{mm}^{2}\right)\end{array}$} & \multicolumn{3}{|c|}{$\overline{\mathbf{H}}(\mathbf{N})$} & \multicolumn{3}{|c|}{$\overline{\mathbf{T}}$ máx (N) } & \multicolumn{3}{|c|}{$\overline{\overline{\mathbf{Y}}} \mathbf{m a ́ x}(\mathbf{m})$} \\
\hline & & PD & $\mathbf{P A}$ & erro \% & PD & PA & erro \% & PD & $\mathbf{P A}$ & erro \% \\
\hline $\mathrm{A} 1$ & 25,22 & 375422 & 403189 & 7,40 & 545597 & 567945 & 4,10 & 20,148 & 19,842 & $-1,52$ \\
\hline A2 & 81,68 & 581755 & 602157 & 3,51 & 703686 & 722906 & 2,73 & 13,369 & 13,286 & $-0,62$ \\
\hline A3 & 145,16 & 706607 & 723621 & 2,41 & 809956 & 826818 & 2,08 & 11,103 & 11,056 & $-0,42$ \\
\hline A4 & 403,23 & 968226 & 980018 & 1,22 & 1045900 & 1058506 & 1,21 & 8,182 & 8,163 & $-0,23$ \\
\hline A5 & 790,32 & 1162595 & 1171529 & 0,77 & 1228036 & 1237934 & 0,81 & 6,840 & 6,829 & $-0,16$ \\
\hline A6 & 1165,54 & 1278182 & 1285665 & 0,59 & 1337981 & 1346453 & 0,63 & 6,231 & 6,222 & $-0,14$ \\
\hline A7 & 1612,90 & 1373975 & 1380352 & 0,46 & 1429773 & 1437140 & 0,52 & 5,802 & 5,796 & $-0,10$ \\
\hline
\end{tabular}


TABELA D.6 - Comparação entre os resultados do Processo Analítico e Processo dos deslocamento - cabos nivelados, $\ell=40 \mathrm{~m}$, $\mathrm{E}=120 \mathrm{E}+9 \mathrm{~N} / \mathrm{m}^{2}$.

\begin{tabular}{|c|c|c|c|c|c|c|c|c|c|c|c|c|c|}
\hline \multirow{2}{*}{$\begin{array}{l}\text { Nome } \\
\text { do cabo }\end{array}$} & \multirow{2}{*}{$\begin{array}{c}\overline{\bar{p}} \\
(\mathbf{N} / \mathbf{m})\end{array}$} & \multirow{2}{*}{$\begin{array}{c}\text { f } \\
\text { (m) }\end{array}$} & \multirow{2}{*}{$\begin{array}{c}\mathrm{A} \\
\left(\mathrm{mm}^{2}\right)\end{array}$} & \multicolumn{3}{|c|}{$\begin{array}{ll}\overline{\mathbf{H}} & \mathbf{( N )}\end{array}$} & \multicolumn{3}{|c|}{$\overline{\mathbf{T}}$ máx (N) } & \multicolumn{3}{|c|}{$\overline{\mathbf{Y}}$ máx (m) } & \multirow{2}{*}{$\begin{array}{l}\text { Tpd } \\
\text { (N) }\end{array}$} \\
\hline & & & & PD & $\overline{\mathbf{P A}}$ & erro \% & PD & PA & erro $\%$ & PD & $\mathbf{P A}$ & erro \% & \\
\hline B1 & \multirow{3}{*}{2500} & \multirow{3}{*}{4} & 197,49 & 113874 & 114268 & 0,35 & 124144 & 124728 & 0,47 & 4,381 & 4,376 & $-0,11$ & \multirow{3}{*}{100000} \\
\hline B2 & & & 145,16 & 110811 & 111311 & 0,45 & 121341 & 122025 & 0,56 & 4,498 & 4,492 & $-0,13$ & \\
\hline B3 & & & 93,39 & 105410 & 106096 & 0,65 & 116429 & 117287 & 0,74 & 4,720 & 4,713 & $-0,15$ & \\
\hline B4 & \multirow{3}{*}{2500} & \multirow{3}{*}{2} & 326,73 & 188760 & 189375 & 0,33 & 195128 & 195864 & 0,38 & 2,643 & 2,640 & $-0,11$ & \multirow{3}{*}{200000} \\
\hline B5 & & & 258,06 & 180607 & 181307 & 0,39 & 187252 & 188075 & 0,44 & 2,760 & 2,758 & $-0,07$ & \\
\hline B6 & & & 197,49 & 170994 & 171799 & 0,47 & 177998 & 178927 & 0,52 & 2,914 & 2,910 & $-0,14$ & \\
\hline B7 & \multirow{3}{*}{5000} & \multirow{3}{*}{4} & 326,73 & 224109 & 225023 & 0,41 & 244954 & 246242 & 0,53 & 4,450 & 4,444 & $-0,13$ & \multirow{3}{*}{200000} \\
\hline $\mathrm{B} 8$ & & & 258,06 & 218973 & 220064 & 0,50 & 240264 & 241719 & 0,61 & 4,551 & 4,544 & $-0,15$ & \\
\hline B9 & & & 197,49 & 212318 & 213638 & 0,62 & 234215 & 235883 & 0,71 & 4,690 & 4,681 & $-0,19$ & \\
\hline B10 & \multirow{3}{*}{5000} & \multirow{3}{*}{2} & 681,62 & 380355 & 381554 & 0,32 & 392999 & 394441 & 0,37 & 2,623 & 2,621 & $-0,08$ & \multirow{3}{*}{500000} \\
\hline B11 & & & 580,64 & 369445 & 370758 & 0,36 & 382450 & 384008 & 0,41 & 2,700 & 2,697 & $-0,11$ & \\
\hline $\mathrm{B} 12$ & & & 403,22 & 343493 & 345087 & 0,46 & 357442 & 359284 & 0,52 & 2,901 & 2,898 & $-0,10$ & \\
\hline $\mathrm{B} 13$ & \multirow{3}{*}{7500} & \multirow{3}{*}{4} & 488,04 & 336035 & 337410 & $\overline{0,41}$ & 367314 & 369250 & 0,53 & 4,452 & 4,445 & $-0,16$ & \multirow{3}{*}{300000} \\
\hline $\mathrm{B} 14$ & & & 403,22 & 329866 & 331454 & 0,48 & 361679 & 363816 & 0,59 & 4,532 & 4,526 & $-0,13$ & \\
\hline $\bar{B} 15$ & & & 326,73 & 322287 & 324136 & 0,57 & 354780 & 357161 & 0,67 & 4,636 & 4,628 & $-0,17$ & \\
\hline B16 & \multirow{3}{*}{7500} & \multirow{3}{*}{2} & 907,45 & 558409 & 560333 & 0,34 & 577772 & 580063 & 0,40 & 2,679 & 2,677 & $-0,07$ & \multirow{3}{*}{600000} \\
\hline B17 & & & 790,32 & 544002 & 546079 & 0,38 & 563860 & 566306 & 0,43 & 2,750 & 2,747 & $-0,11$ & \\
\hline$\overline{B 18}$ & & & 681,62 & 528214 & 530462 & 0,43 & 548644 & 551262 & 0,48 & 2,831 & 2,828 & $-0,11$ & \\
\hline B19 & \multirow{3}{*}{10000} & \multirow{3}{*}{4} & 681,62 & 449911 & 451680 & 0,39 & 491458 & 493979 & 0,51 & 4,434 & 4,428 & $-0,14$ & \multirow{3}{*}{400000} \\
\hline B20 & & & 580,64 & 443245 & 445244 & 0,45 & 485363 & 488101 & 0,56 & 4,498 & 4,492 & $-0,13$ & \\
\hline B21 & & & 403,22 & 425726 & 428328 & 0,61 & 469419 & 472721 & 0,70 & 4,678 & 4,669 & $-0,19$ & \\
\hline B22 & & & 1306,45 & 754993 & 757450 & 0,33 & 780465 & 783410 & 0,38 & 2,643 & 2,640 & $-0,11$ & \\
\hline B23 & 10000 & 2 & 1165,54 & 739397 & 742018 & 0,35 & 765388 & 768499 & 0,41 & 2,698 & 2,695 & $-0,11$ & 800000 \\
\hline B24 & & & 1032,26 & 722431 & 725232 & 0,39 & 749011 & 752304 & 0,44 & 2,760 & 2,758 & $-0,07$ & \\
\hline
\end{tabular}


TABELA D.7-Comparação entre os resultados do Processo Analítico e Processo dos Deslocamento - cabos nivelados, $\ell=60 \mathrm{~m}$, $\mathrm{E}=120 \mathrm{E}+9 \mathrm{~N} / \mathrm{m}^{2}$.

\begin{tabular}{|c|c|c|c|c|c|c|c|c|c|c|c|c|c|}
\hline \multirow{2}{*}{$\begin{array}{c}\text { Nome } \\
\text { do cabo }\end{array}$} & \multirow{2}{*}{$\begin{array}{c}\overline{\bar{p}} \\
(\mathbf{N} / \mathbf{m})\end{array}$} & \multirow{2}{*}{$\begin{array}{c}\mathbf{f} \\
(\mathrm{m})\end{array}$} & \multirow{2}{*}{$\begin{array}{c}A \\
\left(\mathrm{~mm}^{2}\right)\end{array}$} & \multicolumn{3}{|c|}{$\begin{array}{ll}\overline{\mathbf{H}} & (\mathbf{N})\end{array}$} & \multicolumn{3}{|c|}{$\overline{\mathbf{T}}$ máx (N) } & \multicolumn{3}{|c|}{$\overline{\mathbf{Y}}$ máx (m) } & \multirow{2}{*}{$\begin{array}{l}\text { Tpd } \\
(\mathrm{N})\end{array}$} \\
\hline & & & & PD & $\mathbf{P A}$ & erro \% & PD & PA & erro \% & $\mathbf{P D}$ & $\overline{\mathbf{P A}}$ & erro \% & \\
\hline $\mathrm{B} 25$ & \multirow{3}{*}{2500} & \multirow{3}{*}{6} & 258,06 & 168858 & 169517 & 0,39 & 184426 & 185367 & 0,51 & 6,645 & 6,637 & $-0,12$ & \multirow{3}{*}{150000} \\
\hline $\mathrm{B} 26$ & & & 197,49 & 164579 & 165385 & 0,49 & 180517 & 181597 & 0,60 & 6,813 & 6,802 & $-0,16$ & \\
\hline $\mathrm{B} 27$ & & & 145,16 & 158836 & 159839 & 0,63 & 175296 & 176560 & 0,72 & 7,052 & 7,038 & $-0,20$ & \\
\hline B28 & \multirow{3}{*}{2500} & \multirow{3}{*}{3} & 488,04 & 282928 & 283851 & 0,33 & 292486 & 293592 & 0,38 & 3,967 & 3,963 & $-0,10$ & \multirow{3}{*}{300000} \\
\hline B29 & & & 403,22 & 273064 & 274092 & 0,38 & 282956 & 284168 & 0,43 & 4,108 & 4,104 & $-0,10$ & \\
\hline B30 & & & 326,73 & 261827 & 262976 & 0,44 & 272128 & 273462 & 0,49 & 4,283 & 4,278 & $-0,12$ & \\
\hline B31 & \multirow{3}{*}{5000} & \multirow{3}{*}{6} & 488,04 & 336035 & 337410 & 0,41 & 367314 & 369250 & 0,53 & 6,677 & 6,668 & $-0,13$ & \multirow{3}{*}{300000} \\
\hline B32 & & & 403,22 & 329866 & 331454 & 0,48 & 361593 & 363816 & 0,61 & 6,799 & 6,788 & $-0,16$ & \\
\hline B33 & & & 326,73 & 322287 & 324131 & 0,57 & 354780 & 357161 & 0,67 & 6,954 & 6,941 & $-0,19$ & \\
\hline $\mathrm{B} 34$ & \multirow{3}{*}{5000} & \multirow{3}{*}{3} & 907,45 & $5 \overline{8409}$ & 560333 & 0,34 & 577772 & 580063 & 0,40 & 4,019 & 4,015 & $-0,10$ & \multirow{3}{*}{600000} \\
\hline $\mathrm{B} 35$ & & & 790,32 & 544002 & 546079 & 0,38 & 563860 & 566306 & 0,43 & 4,124 & 4,120 & $-0,10$ & \\
\hline$\overline{B 36}$ & & & 681,62 & 528214 & 530462 & 0,43 & 548644 & 551262 & 0,48 & 4,246 & 4,242 & $-0,09$ & \\
\hline B37 & \multirow{3}{*}{7500} & \multirow{3}{*}{6} & 681,62 & 500710 & 502888 & 0,43 & 547915 & 550928 & 0,55 & 6,721 & 6,711 & $-0,15$ & \multirow{3}{*}{450000} \\
\hline B38 & & & 580,64 & 492690 & 495144 & 0,50 & 540595 & 543868 & 0,61 & 6,827 & 6,816 & $-0,16$ & \\
\hline B39 & & & 488,04 & 483191 & 485972 & 0,58 & 531953 & 535531 & 0,67 & 6,957 & 6,945 & $-0,17$ & \\
\hline B40 & \multirow{3}{*}{7500} & \multirow{3}{*}{3} & 1465,07 & 848881 & 851651 & 0,33 & 877553 & 880871 & 0,38 & 3,966 & 3,963 & $-0,08$ & \multirow{3}{*}{900000} \\
\hline B41 & & & 1306,45 & 831253 & 834208 & 0,36 & 860513 & 864018 & 0,41 & 4,050 & 4,046 & $-0,10$ & \\
\hline $\mathrm{B} 42$ & & & 1165,54 & 813314 & 816459 & 0,39 & 843196 & 846895 & 0,44 & 4,138 & 4,134 & $-0,10$ & \\
\hline B43 & \multirow{3}{*}{10000} & \multirow{3}{*}{6} & 907,45 & 667517 & 670424 & 0,44 & 730465 & 734485 & 0,55 & 6,722 & 6,712 & $-0,15$ & \multirow{3}{*}{600000} \\
\hline B44 & & & 790,32 & 658349 & 661572 & 0,49 & 722096 & 726414 & 0,60 & 6,813 & 6,802 & $-0,16$ & \\
\hline B45 & & & 681,62 & 647746 & 651334 & 0,55 & 712443 & 717103 & 0,65 & 6,921 & 6,909 & $-0,17$ & \\
\hline B46 & & & 1612,90 & 1092260 & 1096369 & 0,38 & 1131826 & 1136673 & 0,43 & 4,108 & 4,104 & $-0,10$ & \\
\hline B47 & 10000 & 3 & 1465,07 & 1071885 & 1076214 & 0,40 & 1112177 & 1117245 & 0,46 & 4,186 & 4,181 & $-0,12$ & 1200000 \\
\hline B48 & & & 1306,45 & 1047230 & 1051828 & 0,44 & 1088435 & 1093774 & 0,49 & 4,283 & 4,278 & $-0,12$ & \\
\hline
\end{tabular}


TABELA D.8-Comparação entre os resultados do Processo Analítico e Processo dos deslocamento - cabos nivelados, $\ell=80 \mathrm{~m}$, $\mathrm{E}=120 \mathrm{E}+9 \mathrm{~N} / \mathrm{m}^{2}$.

\begin{tabular}{|c|c|c|c|c|c|c|c|c|c|c|c|c|c|}
\hline \multirow{2}{*}{$\begin{array}{c}\text { Nome } \\
\text { do cabo }\end{array}$} & \multirow{2}{*}{$\begin{array}{c}\overline{\overline{\mathbf{p}}} \\
(\mathrm{N} / \mathrm{m})\end{array}$} & \multirow{2}{*}{$\begin{array}{c}f \\
\text { (m) }\end{array}$} & \multirow{2}{*}{$\begin{array}{c}A \\
\left(\mathrm{~mm}^{2}\right)\end{array}$} & \multicolumn{3}{|c|}{$\begin{array}{ll}\overline{\mathbf{H}} & (\mathrm{N}) \\
\end{array}$} & \multicolumn{3}{|c|}{$\overline{\mathrm{T}} \operatorname{máx}(\mathrm{N})$} & \multicolumn{3}{|c|}{$\overline{\mathbf{Y}}$ máx (m) } & \multirow{2}{*}{$\begin{array}{l}\text { Tpd } \\
(\mathrm{N})\end{array}$} \\
\hline & & & & PD & $\overline{\mathbf{P A}}$ & erro \% & PD & PA & erro \% & PD & PA & erro \% & \\
\hline B49 & \multirow{3}{*}{2500} & \multirow{3}{*}{8} & 326,73 & 224109 & 225023 & 0,41 & 244991 & 246242 & 0,51 & 8,900 & 8,888 & $-0,13$ & \multirow{3}{*}{200000} \\
\hline B50 & & & 258,06 & 218973 & 220064 & 0,50 & 240302 & 241719 & 0,59 & 9,103 & 9,088 & $-0,16$ & \\
\hline B51 & & & 197,49 & 212318 & 213638 & 0,62 & 234254 & 235883 & 0,70 & 9,379 & 9,362 & $-0,18$ & \\
\hline B52 & \multirow{3}{*}{2500} & \multirow{3}{*}{4} & 681,62 & 380356 & 381554 & 0,31 & 393023 & 394441 & 0,36 & 5,246 & 5,242 & $-0,08$ & \multirow{3}{*}{400000} \\
\hline B53 & & & 580,64 & 369446 & 370758 & 0,36 & 382474 & 384008 & 0,40 & 5,399 & 5,394 & $-0,09$ & \\
\hline$\overline{B 54}$ & & & 403,22 & 343494 & 345087 & 0,46 & 357469 & 359284 & 0,51 & 5,802 & 5,796 & $-0,10$ & \\
\hline B55 & \multirow{3}{*}{5000} & \multirow{3}{*}{8} & 681,62 & 449911 & 451680 & 0,39 & 491531 & 493979 & 0,50 & 8,867 & 8,856 & $-0,12$ & \multirow{3}{*}{400000} \\
\hline B56 & & & 580,64 & 443245 & 445244 & 0,45 & 485438 & 488101 & 0,55 & 8,997 & 8,984 & $-0,14$ & \\
\hline B57 & & & 403,22 & 425726 & 428328 & 0,61 & 469496 & 472721 & 0,69 & 9,356 & 9,339 & $-0,18$ & \\
\hline B58 & \multirow{3}{*}{5000} & \multirow{3}{*}{4} & 1306,45 & 754994 & 757450 & 0,33 & 780513 & 783410 & 0,37 & 5,285 & 5,281 & $-0,08$ & \multirow{3}{*}{800000} \\
\hline B59 & & & 1165,54 & 739399 & 742018 & 0,35 & 765437 & 768499 & 0,40 & 5,396 & 5,391 & $-0,09$ & \\
\hline B60 & & & 1032,26 & 722432 & 725232 & 0,39 & 749061 & 752304 & 0,43 & 5,521 & 5,515 & $-0,11$ & \\
\hline B61 & \multirow{3}{*}{7500} & \multirow{3}{*}{8} & 907,45 & 667516 & 670424 & 0,44 & 730576 & 734485 & 0,54 & 8,962 & 8,950 & $-0,13$ & \multirow{3}{*}{600000} \\
\hline B62 & & & 790,32 & 658348 & 661572 & 0,49 & 722209 & 726414 & 0,58 & 9,083 & 9,069 & $-0,15$ & \\
\hline B63 & & & 681,62 & 647746 & 651334 & 0,55 & 712558 & 717103 & 0,64 & 9,228 & 9,212 & $-0,17$ & \\
\hline B72 & \multirow{3}{*}{7500} & \multirow{3}{*}{4} & 1612,90 & 1092262 & 1096369 & 0,38 & 1131901 & 1136673 & 0,42 & 5,478 & 5,473 & $-0,09$ & \multirow{3}{*}{1200000} \\
\hline B65 & & & 1465,07 & 1071887 & 1076214 & 0,40 & 1112253 & 1117245 & 0,45 & 5,581 & 5,575 & $-0,11$ & \\
\hline B66 & & & 1306,45 & 1047233 & 1051828 & 0,44 & 1088513 & 1093774 & 0,48 & 5,711 & 5,704 & $-0,12$ & \\
\hline B67 & \multirow{3}{*}{10000} & \multirow{3}{*}{8} & 1306,45 & 896405 & 900062 & 0,41 & 979937 & 984943 & 0,51 & 8,900 & 8,888 & $-0,13$ & \multirow{3}{*}{800000} \\
\hline$\overline{B 68}$ & & & 1165,54 & 886803 & 890796 & 0,45 & 971166 & 976482 & 0,55 & 8,994 & 8,981 & $-0,14$ & \\
\hline$\overline{\mathrm{B} 69}$ & & & 1032,26 & 875893 & 880257 & 0,50 & 961210 & 966878 & 0,59 & 9,103 & 9,088 & $-0,16$ & \\
\hline B70 & & & 1612,90 & 1373979 & 1380352 & 0,46 & 1429878 & 1437140 & 0,51 & 5,802 & 5,796 & $-0,10$ & \\
\hline B71 & 10000 & 4 & 1465,07 & 1345848 & 1352539 & 0,50 & 1402870 & 1410448 & 0,54 & 5,922 & 5,915 & $-0,12$ & 1600000 \\
\hline & & & 1306,45 & 1312050 & 1319130 & 0,54 & 1370478 & 1378442 & 0,58 & 6,072 & 6,065 & $-0,12$ & \\
\hline
\end{tabular}


TABELA D. 9 - Comparação entre os resultados do Processo Analítico e Processo dos deslocamentos - cabos desnivelados com $\mathrm{E}=120 \mathrm{E}+9 \mathrm{~N} / \mathrm{m}^{2}$.

\begin{tabular}{|c|c|c|c|c|c|c|c|c|c|c|c|c|}
\hline \multirow{2}{*}{$\begin{array}{l}\text { Nome } \\
\text { do cabo }\end{array}$} & \multirow{2}{*}{$\begin{array}{c}\mathrm{L} \\
\text { (m) }\end{array}$} & \multirow{2}{*}{$\begin{array}{c}\overline{\mathbf{p}} \\
(\mathbf{N} / \mathbf{m})\end{array}$} & \multirow{2}{*}{$\begin{array}{c}\mathbf{f} \\
\text { (m) }\end{array}$} & \multirow{2}{*}{$\begin{array}{c}\text { h } \\
\text { (m) }\end{array}$} & \multirow{2}{*}{$\begin{array}{c}A \\
\left(\mathrm{~mm}^{2}\right)\end{array}$} & \multicolumn{3}{|c|}{$\begin{array}{ll}\overline{\mathbf{H}} & (\mathbf{N})\end{array}$} & \multicolumn{3}{|c|}{$\overline{\mathbf{T}} \mathbf{m} \mathbf{x} \mathbf{x} \quad$ (N) } & \multirow{2}{*}{$\begin{array}{l}\text { Tpd } \\
\text { (N) }\end{array}$} \\
\hline & & & & & & $\overline{\mathbf{P D}}$ & $\overline{\mathbf{P A}}$ & erro \% & PD & $\overline{\mathbf{P A}}$ & erro \% & \\
\hline$\overline{\mathrm{C}} 1$ & 40 & & 4 & -10 & 133,5 & 106437 & 106971 & 0,50 & 164360 & 165851 & 0,91 & 88900 \\
\hline C2 & 60 & 5000 & 6 & -15 & 195,0 & 159482 & 160303 & 0,51 & 246602 & 248648 & 0,83 & 130000 \\
\hline$\overline{\mathrm{C} 3}$ & 80 & & 8 & -20 & 270,0 & 212974 & 214027 & 0,49 & 329422 & 331773 & 0,71 & 180000 \\
\hline
\end{tabular}

\begin{tabular}{|l|l|l|l|l|l|l|l|l|l|l|l|l|}
\hline C4 & 60 & & 6 & -5 & 317,6 & 246268 & 247454 & 0,48 & 298533 & 300575 & 0,68 & 211700 \\
\hline C5 & 60 & & 6 & -2 & 385,7 & 292538 & 293874 & 0,46 & 332444 & 334509 & 0,62 & 257100 \\
\hline C6 & 60 & 5000 & 6 & 0 & 450,0 & 333495 & 334958 & 0,44 & 364906 & 367010 & 0,58 & 300000 \\
\hline C7 & 60 & & 6 & 2 & 540,0 & 386428 & 388097 & 0,43 & 409387 & 411589 & 0,54 & 360000 \\
\hline C8 & 60 & & 6 & 5 & 771,4 & 501685 & 504055 & 0,47 & 512776 & 515494 & 0,53 & 514200 \\
\hline
\end{tabular}


TABELA D.10 - Comparação entre as expressões originais e aproximadas empregadas no Processo Analítico.

\begin{tabular}{|c|c|c|c|c|c|}
\hline Nome do cabo & Nó & E1\% & E2\% & E3\% & E4\% \\
\hline \multirow{5}{*}{ B31 } & 1 & $-1,15 \mathrm{E}+00$ & $-6,27 \mathrm{E}-01$ & $5,76 \mathrm{E}-01$ & $3,02 \mathrm{E}-03$ \\
\hline & 2 & $1,43 \mathrm{E}-01$ & $-5,87 \mathrm{E}-01$ & $4,22 \mathrm{E}-02$ & $1,97 \mathrm{E}-03$ \\
\hline & 3 & $5,76 \mathrm{E}-01$ & $-5,73 \mathrm{E}-01$ & $0,00 \mathrm{E}+00$ & $1,65 \mathrm{E}-03$ \\
\hline & 4 & $1,43 \mathrm{E}-01$ & $-5,87 \mathrm{E}-01$ & $4,22 \mathrm{E}-02$ & $1,97 \mathrm{E}-03$ \\
\hline & 5 & $-1,15 E+00$ & $-6,27 \mathrm{E}-01$ & $5,76 \mathrm{E}-01$ & $3,02 \mathrm{E}-03$ \\
\hline \multirow{5}{*}{ B32 } & 1 & $-1,37 \mathrm{E}+00$ & $-7,46 \mathrm{E}-01$ & $6,46 \mathrm{E}-01$ & $4,54 \mathrm{E}-03$ \\
\hline & 2 & $1,70 \mathrm{E}-01$ & $-6,97 \mathrm{E}-01$ & $4,78 \mathrm{E}-02$ & $2,85 \mathrm{E}-03$ \\
\hline & 3 & $6,85 \mathrm{E}-01$ & $-6,80 \mathrm{E}-01$ & $0,00 \mathrm{E}+00$ & $2,33 \mathrm{E}-03$ \\
\hline & 4 & $1,70 \mathrm{E}-01$ & $-6,97 \mathrm{E}-01$ & $4,78 \mathrm{E}-02$ & $2,85 \mathrm{E}-03$ \\
\hline & 5 & $-1,37 E+00$ & $-7,46 \mathrm{E}-01$ & $6,46 \mathrm{E}-01$ & $4,54 \mathrm{E}-03$ \\
\hline \multirow{5}{*}{ B33 } & 1 & $-1,65 E+00$ & $-9,03 \mathrm{E}-01$ & $7,44 \mathrm{E}-01$ & $7,12 \mathrm{E}-03$ \\
\hline & 2 & $2,06 \mathrm{E}-01$ & $-8,41 \mathrm{E}-01$ & $5,57 \mathrm{E}-02$ & $4,26 \mathrm{E}-03$ \\
\hline & 3 & $8,27 \mathrm{E}-01$ & $-8,20 \mathrm{E}-01$ & $0,00 \mathrm{E}+00$ & $3,39 \mathrm{E}-03$ \\
\hline & 4 & $2,06 \mathrm{E}-01$ & $-8,41 \mathrm{E}-01$ & $5,57 \mathrm{E}-02$ & $4,26 \mathrm{E}-03$ \\
\hline & 5 & $-1,65 E+00$ & $-9,03 \mathrm{E}-01$ & $7,44 \mathrm{E}-01$ & $7,12 \mathrm{E}-03$ \\
\hline \multirow{5}{*}{ B34 } & 1 & $-1,03 E+00$ & $-5,30 \mathrm{E}-01$ & $1,42 \bar{E}-01$ & $4,43 \mathrm{E}-03$ \\
\hline & 2 & $1,29 \mathrm{E}-01$ & $-5,16 \mathrm{E}-01$ & $9,62 \mathrm{E}-03$ & $1,95 \mathrm{E}-03$ \\
\hline & 3 & $5,15 \mathrm{E}-01$ & $-5,12 \mathrm{E}-01$ & $0,00 \mathrm{E}+00$ & $1,32 \mathrm{E}-03$ \\
\hline & 4 & $1,29 \mathrm{E}-01$ & $-5,16 \mathrm{E}-01$ & $9,62 \mathrm{E}-03$ & $1,95 \mathrm{E}-03$ \\
\hline & 5 & $-1,03 E+00$ & $-5,30 \mathrm{E}-01$ & $1,42 \mathrm{E}-01$ & $4,43 \mathrm{E}-03$ \\
\hline \multirow{5}{*}{ B35 } & 1 & $-1,15 \mathrm{E}+00$ & $-5,94 \mathrm{E}-01$ & $1,66 \mathrm{E}-01$ & $5,95 \mathrm{E}-03$ \\
\hline & 2 & $1,44 \mathrm{E}-01$ & $-5,78 \mathrm{E}-01$ & $1,13 \mathrm{E}-02$ & $2,51 \mathrm{E}-03$ \\
\hline & 3 & $5,76 \mathrm{E}-01$ & $-5,73 \mathrm{E}-01$ & $0,00 \mathrm{E}+00$ & $1,65 \mathrm{E}-03$ \\
\hline & 4 & $1,44 \mathrm{E}-01$ & $-5,78 \mathrm{E}-01$ & $1,13 \mathrm{E}-02$ & $2,51 \mathrm{E}-03$ \\
\hline & 5 & $-1,15 E+00$ & $-5,94 \mathrm{E}-01$ & $1,66 \mathrm{E}-01$ & $5,95 \mathrm{E}-03$ \\
\hline \multirow{5}{*}{ B36 } & 1 & $-1,30 \mathrm{E}+00$ & $-6,69 \mathrm{E}-01$ & $1,97 \mathrm{E}-01$ & $8,13 \mathrm{E}-03$ \\
\hline & 2 & $1,62 \mathrm{E}-01$ & $-6,51 \mathrm{E}-01$ & $1,35 \mathrm{E}-02$ & $3,28 \mathrm{E}-03$ \\
\hline & 3 & $6,49 \mathrm{E}-01$ & $-6,44 \mathrm{E}-01$ & $0,00 \mathrm{E}+00$ & $2,09 \mathrm{E}-03$ \\
\hline & 4 & $1,62 \mathrm{E}-01$ & $-6,51 \mathrm{E}-01$ & $1,35 \mathrm{E}-02$ & $3,28 \mathrm{E}-03$ \\
\hline & 5 & $-1,30 E+00$ & $-6,69 \mathrm{E}-01$ & $1,97 \mathrm{E}-01$ & $8,13 \mathrm{E}-03$ \\
\hline
\end{tabular}

